\title{
Weakest Preexpectation Semantics for Bayesian Inference ${ }^{\star}$ \\ Conditioning, Continuous Distributions and Divergence
}

\author{
Marcin Szymczak and Joost-Pieter Katoen \\ Software Modelling and Verification Group \\ RWTH Aachen University \\ 52056 Aachen, Germany
}

\begin{abstract}
We present a semantics of a probabilistic while-language with soft conditioning and continuous distributions which handles programs diverging with positive probability. To this end, we extend the probabilistic guarded command language (pGCL) with draws from continuous distributions and a score operator. The main contribution is an extension of the standard weakest preexpectation semantics to support these constructs. As a sanity check of our semantics, we define an alternative trace-based semantics of the language, and show that the two semantics are equivalent. Various examples illustrate the applicability of the semantics.
\end{abstract}

\section{Introduction}

Research on semantics of probabilistic languages for machine learning $6,28,37$, 15, 5] has so far focused almost exclusively on almost-surely terminating programs. These programs terminate on all possible inputs with probability one. This seems a reasonable assumption, because not only most probabilistic models used in practice terminate with probability one, but programs which may diverge with a positive probability also make not much sense in the context of probabilistic inference.

However, one cannot simply assume that in the context of statistical probabilistic programming, divergence is a non-existing issue which can be ignored completely. For one thing, models which are not guaranteed to terminate actually exist, and are not merely degenerate cases. Even if one cannot apply inference in this case, being able to reason about such programs is important, as it helps to define suitable approximations and check their correctness. Moreover, the line dividing almost surely terminating and possibly diverging programs can sometimes be very thin and a small change to some parameter values may make a terminating program diverge.

To make a case for potentially diverging probabilistic programs, consider a variation of the tortoise and hare problem described by Icard [18] as a simple

\footnotetext{
* This work is supported by the ERC Advanced Grant Project FRAPPANT (project number 787914).
} 
problem in intuitive physics: a tortoise is walking at some low constant speed and a hare, which was initially behind the tortoise and moves forward with random, fast strides, is trying to catch it. Assuming that the tortoise is moving at a constant speed of $1+\mathrm{e} \mathrm{cm}$ per second (where e is some small constant), and each second the hare moves with probability $\frac{1}{4}$ by a random Gaussian-distributed distance, being $4 \mathrm{~cm}$ on average, we would like to calculate the average time after which the hare will catch the tortoise. We can model this problem by the following probabilistic program:

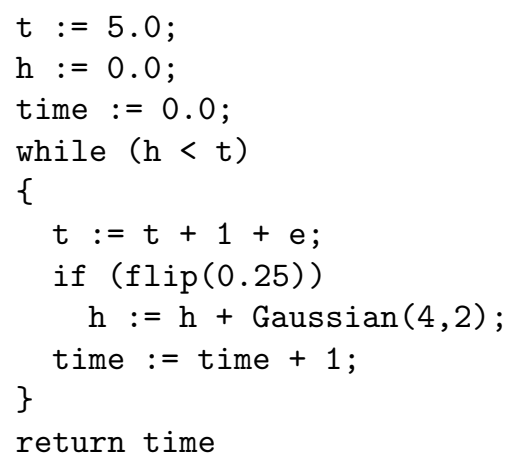

where $\mathrm{flip}(p)$ returns true with probability $p$ and false with probability $1-$ $p$ and Gaussian $(\mu, \sigma)$ draws a random value from the Gaussian distribution with mean $\mu$ and variance $\sigma$. It can be proven that if $\mathrm{e}=0$, the program terminates with probability one, but if e $>0$, the program may diverge with positive probability, no matter how small e is. In other words, if the tortoise moves at a speed strictly greater than one, the hare may never catch it.

The above program is a simple forward simulation, which does not use conditioning at all. However, we may also invert the problem and ask what was the tortoise's head start given that the hare caught the tortoise in around one minute. This could be modelled by the following probabilistic program:

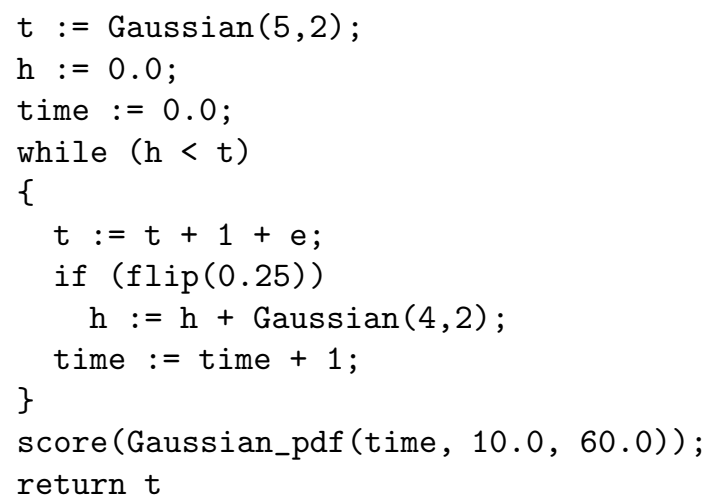

where score intuitively multiplies the probability of the current program run by its argument, and Gaussian_pdf $(\mu, \sigma, x)=\frac{1}{\sqrt{2 \pi} \sigma} e^{-\frac{1}{2 \sigma^{2}}(\mu-x)^{2}}$ is the value 
of the density function of the Gaussian distribution with mean $\mu$ and variance $\sigma$ at point $x$. Now, although we assume that the hare caught the tortoise, the program may still diverge with a positive probability. In order to reason about programs like this, we need a framework which supports soft conditioning - as modelled by score in our setting - and is able to handle diverging programs.

As a more complicated example, let us consider the inverse intuitive physics example from [12]1. In this model, using noisy approximate Newtonian dynamics, a ball is falling on the ground from a certain height, potentially hitting some fixed obstacles on the way. Given the observed final position of the ball, we want to find the distribution on initial locations of the ball. Similarly to the above example, this model is implemented as a simulation of the ball's movement from the random initial position (sampled from the prior), followed by soft conditioning on the ball's final position. Depending on the shapes and locations of the obstacles and the size of the floor on which the ball is supposed to land, the program may not terminate - the ball may get stuck in the air, blocked by obstacles, or may fail to land on the floor and keep falling indefinitely.

Issues with program divergence may also appear when implementing models which are not designed to be possibly diverging - be it because of mistakes in the implementation or intricacies and subtleties of the model itself. For instance, the implementation of the Pitman-Yor process 19] on forestdb.org, an online repository of probabilistic models in Church [10] and WebPPL [11], occasionally fails. According to a note on the website, a possible cause is that the program may not almost surely terminate.

Another issue related to program divergence is that some implementations of sampling-based algorithms do not handle detected divergence correctly —instead of throwing an appropriate error message, they simply ignore diverging runs after a given number of steps, which leads to misleading inference results. For instance, consider the following WebPPL program taken from [30]:

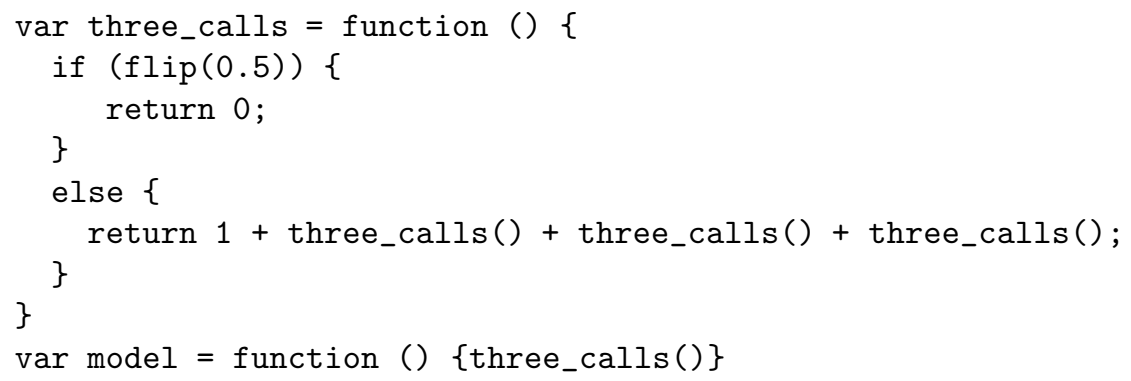

This program does not almost surely terminate and its expected outcome is infinite. However, applying WebPPL's enumeration (exact) inference algorithm with a bounded maximum number of executions to this program gives a distribution assigning a probability of over 0.8 to outcome 0 and minuscule probabilities to other outcomes. No warning about the maximum number of evaluation steps being reached is given.

\footnotetext{
${ }^{1}$ available online under http://probmods.org/chapters/conditioning.html.
} 
There has been research on the semantics of non-terminating probabilistic programs [27, 26, 13]. However, this research was mostly aimed at analysing randomised algorithms, rather than Bayesian inference. As a consequence, most languages used in this line of research have no features such as continuous distributions and soft conditioning, which are the cornerstone of Bayesian probabilistic programming. While some authors consider non-terminating programs in the context of Bayesian reasoning [20, 29, 21], they normally restrict their attention to discrete programs with hard conditioning by means of Boolean predicates. So far, to our knowledge, the only work which comprehensively treats non-termination in the context of semantics of Bayesian probabilistic programming with continuous distributions is [3]. This paper defines a semantics which calculates the probability of divergence and the probability of failing a hard constraint explicitly. Soft constraints are not considered for diverging programs, as the authors argue that the probability of divergence normalised by soft constraints may be undefined for some programs if unbounded scores are allowed. The authors do not attempt to restrict the language so that scores would make sense for diverging programs.

In this paper, we investigate how the addition of continuous distributions and soft conditioning, necessary for most machine learning applications, affects the semantics of potentially diverging procedural probabilistic programs. We discuss why dealing with divergence in programs with soft conditioning is very difficult (if at all possible) and why one cannot expect any sampling-based semantics to fully correspond to the intuitive meaning of a potentially diverging program. Nevertheless, we also aim to define the first semantics of a probabilistic language supporting both continuous distributions and hard and soft conditioning which is designed to handle diverging programs. We discuss the strengths and limitations of this semantics and state in what sense it can be considered correct.

We provide both a denotational weakest preexpectation semantics à la Kozen [24] and McIver and Morgan [26] together with an operational sampling-based semantics, and prove that the two semantics are equivalent. Hence, this paper extends the standard weakest preexpectation framework to programs with continuous distributions and soft conditioning while being able to treat program divergence.

\section{A Bayesian probabilistic while-language}

We start off by presenting the syntax of a simple probabilistic while-language, simply called PL, which will be used throughout this paper. Besides the usual ingredients such as skip and diverge statements, assignments, sequential composition, conditional statements and guarded loops, the language contains three additional constructs: (a) random draws from continuous distributions, (b) observations encoding hard conditioning, and (c) a score function used for soft conditioning. These forms of conditioning are central to Bayesian inference.

The syntax is presented in Fig. 11 where $C, C_{1}$, and $C_{2}$ are programs, $x$ is a program variable, $U$ denotes the continuous uniform distribution on the unit interval, $\phi$ is a predicate over the program variables, and $E$ is an arithmetic 


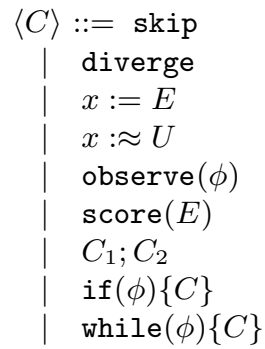

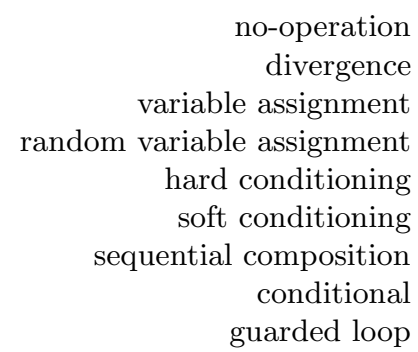

no-operation ariable assignment hard conditioning soft conditioning guarded loop

Fig. 1. Syntax of PL

expression over the program variables. We do not specify the syntax of expressions $E$ and predicates $\phi$-we assume these may be arbitrary, as long as the corresponding evaluation functions are measurable (as explained later).

A few remarks concerning the syntax are in order. In order to simplify the approximation of while loops (as used later), we use the if operator without an else clause. This does not change the expressiveness of the language. For the same reason, the explicit diverge statement is used as syntactic sugar for while(true)\{skip\}. In random assignments, we only allow sampling from the uniform distribution $U$ on the unit interval $[0,1]$. This does not limit the expressiveness of the language, as samples from an arbitrary continuous distribution can be obtained by sampling from the unit interval and applying the inverse cumulative distribution function (inverse cdf) of the given distribution to the generated sample. For instance, we can generate a sample from the Gaussian distribution with mean mu and variance sigma as follows

$\mathrm{u}:=\mathrm{U}$;

$\mathrm{x}:=$ Gaussian_inv_cdf $(\mathrm{mu}, \mathrm{sigma}, \mathrm{u})$;

where Gaussian_inv_cdf $(\mu, \sigma, u)$ returns the value of the inverse cumulative distribution function of the Gaussian distribution with mean $\mu$ and variance $\sigma$ at point $u$-in other words, Gaussian_inv_cdf $(\mu, \sigma, u)$ is a value $v \in \mathbb{R}$ such that $\int_{-\infty}^{v}$ Gaussian_pdf $(\mu, \sigma, x) d x=u$.

Random draws from discrete probability distributions can also be encoded by uniform draws from the unit interval, see e.g. 31]. For instance, the statement if $(f \operatorname{lip}(0.25))\{C\}$ as used in the introduction is a shorthand for $\mathrm{u}: \approx$ $\mathrm{U} ;$ if $(\mathrm{u}<0.25)\{C\}$.

Let us briefly describe the semantics of the three new syntactic constructs at an intuitive level; the rest of this paper is devoted to make this precise. The execution of the random variable assignment $x: \approx U$ incorporates taking a sample from the uniform distribution $U$ and assigning this sample to the program variable $x$. The observe $(\phi)$ statement is similar to the assert $(\phi)$ statement: it

\footnotetext{
${ }^{2}$ Note that the value of Gaussian_inv_cdf $(\mu, \sigma, u)$ is technically only defined for $u \in(0,1)$, but we can safely extend it to $[0,1]$ by setting Gaussian_inv_cdf $(\mu, \sigma, 0)$ and Gaussian_inv_cdf $(\mu, \sigma, 1)$ to some arbitrary value (say, 0 ), as the probability of drawing 0 or 1 from the continuous uniform distribution on $[0,1]$ is zero, anyway.
} 
has no effect for program runs satisfying the predicate $\phi$, but program runs violating $\phi$ are invalid. Such invalid runs are discontinued (aka: stopped). The crucial difference to the assert statement is that probabilities of valid program runs are normalised with respect to the total probability mass of all valid runs. For instance, the only valid runs of program

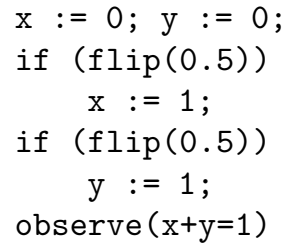

are $\mathrm{x}=0, \mathrm{y}=1$ and $\mathrm{x}=1, \mathrm{y}=0$. Although in absence of the observe-statement the probability of each such run is $1 / 4$, their probability now becomes $1 / 2$ due to normalising $1 / 4$ with the probability of obtaining a valid run, i.e., 1/2. (As discussed extensively in [29], the semantics becomes more tricky when program divergences are taken into account.) As runs are abandoned that violate the predicate $\phi$, this is called hard conditioning.

In contrast, the statement $\mathrm{score}(E)$ models soft conditioning. As effect of executing this statement the probability of the current program run is scaled (i.e. multiplied) by the current value of the expression $E$. The higher the value of $E$, the more likely the combination of random variables sampled so far is considered to be.

To illustrate how soft conditioning works, suppose that we have a function softeq $(a, b)=e^{-(a-b)^{2}}$, whose value is 1 if both arguments are the same and moves closer to 0 as the arguments move further apart. Now, consider the following program:

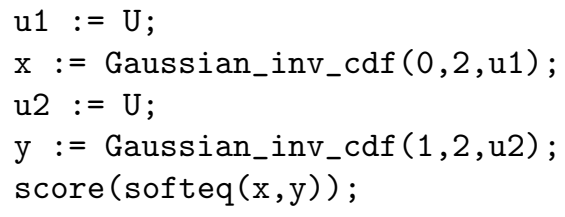

The use of score has the effect that program runs in which $\mathrm{x}$ and $\mathrm{y}$ are closer to each other are more likely.

\section{Denotational semantics}

We will now define the semantics of PL in a weakest precondition style manner. This semantics builds upon the semantics of the probabilistic guarded command language pGCL 25] extended with hard conditioning as defined in [29]. The key object wp $\llbracket C \rrbracket(f)(\sigma)$ defines the expected value of a function $f$ with respect to the probability distribution of final states of program $C$, provided the program starts in the initial state $\sigma$. The key difference to [25, 29] is that dealing with 
continuous distributions requires some sort of integration, and the integrated functions must be well-behaved.

Defining a denotational semantics of a language allowing unbounded computations requires the use of domain theory, which helps to ensure that all semantic functions used are well-defined. Some basic definitions from domain theory, which are needed to understand this paper, are included in Appendix B.

Probability theory with continuous random variables is usually formalised using measure theory and the semantics of PL follows this route. For the sake of completeness, the main relevant ingredients of measure theory are summarised in Appendix A. We start off by defining a measurable state space, and the domain of measurable expectations - the quantitative analogue of predicates. After shortly defining the (standard) semantics of expressions and predicates, we define a weakest preexpectation semantics of PL and subsequently generalise this towards a weakest liberal preexpectation semantics that takes program divergence explicitly into account.

\subsection{Measurable space of states}

In the same vein as [25, 29], the semantics wp $\llbracket C \rrbracket(f)(\sigma)$ will be defined as the expected value of the measurable function $f$ mapping states to nonnegative reals (extended with $\infty$ ). In order to reason about measurable functions on states, we first define a measurable space of program states.

Let $\mathcal{N}$ be a countable set of variable names ranged over by $x_{i}$. A program state maps program variables to their current value. Formally, state $\sigma$ is a set $\left\{\left(x_{1}, V_{1}\right), \ldots,\left(x_{n}, V_{n}\right)\right\}$ of pairs of unique variable names $x_{i}$ and their corresponding values $V_{i} \in \mathbb{R}$. The set $\Omega_{\sigma}$ has the following form:

$$
\Omega_{\sigma}=\biguplus_{n \in \mathbb{N}}\left(\left\{\left\{\left(x_{1}, V_{1}\right), \ldots,\left(x_{n}, V_{n}\right)\right\} \mid \forall i \in 1 . . n x_{i} \in \mathcal{N}, V_{i} \in \mathbb{R} . \forall j \neq i x_{i} \neq x_{j}\right\}\right)
$$

The state space $\Omega_{\sigma}$ is equipped with the functions: $\operatorname{dom}(\cdot): \Omega_{\sigma} \rightarrow P(\mathcal{N})$, returning the domain of a state (i.e., the set of variables which are assigned values), and elem $(\cdot, \cdot): \Omega_{\sigma} \times \mathcal{N} \rightarrow \mathbb{R} \uplus\{\perp\}$ such that elem $(\sigma, x)$ (for convenience, abbreviated $\sigma(x))$ returns the value assigned to variable $x$ in $\sigma$ or $\perp$ if $x \notin \operatorname{dom}(\sigma)$. The functions dom and elem are defined for $\sigma=\left\{\left(x_{1}, V_{1}\right), \ldots,\left(x_{n}, V_{n}\right)\right\}$ as:

$$
\begin{aligned}
\operatorname{dom}(\sigma) & =\left\{x_{1}, \ldots, x_{n}\right\} \\
\text { elem }(\sigma, y)=\sigma(y) & = \begin{cases}V_{i} & \text { if } y=x_{i} \text { for some } i \\
\perp & \text { otherwise }\end{cases}
\end{aligned}
$$

Let the metric $d_{\sigma}$ on $\Omega_{\sigma}$ be defined as follows:

$$
d_{\sigma}\left(\sigma_{1}, \sigma_{2}\right)= \begin{cases}\sum_{x \in \operatorname{dom}\left(\sigma_{1}\right)}\left|\sigma_{1}(x)-\sigma_{2}(x)\right| & \text { if } \operatorname{dom}\left(\sigma_{1}\right)=\operatorname{dom}\left(\sigma_{2}\right) \\ \infty & \text { otherwise }\end{cases}
$$

It is easy to verify that $d_{\sigma}$ is indeed a metric. Note that on the subset of states with a fixed domain $\left\{x_{1}, \ldots, x_{n}\right\}, d_{\sigma}$ is essentially the Manhattan distance. 
Lemma 1. The metric space $\left(\Omega_{\sigma}, d_{\sigma}\right)$ is separable.

Proof. Consider a subset $\Omega_{\sigma}^{\mathbb{Q}}$ of $\Omega_{\sigma}$ where all values are rational. Then the set $\Omega_{\sigma}^{\mathbb{Q}}$ is countable and it can be easily verified that it is a dense subset of $\Omega_{\sigma}$. Hence, $\left(\Omega_{\sigma}, d_{\sigma}\right)$ is separable.

Finally, let $\Sigma_{\sigma}$ be the Borel $\sigma$-algebra on $\Omega_{\sigma}$ induced by the metric $d_{\sigma}$. The pair $\left(\Omega_{\sigma}, \Sigma_{\sigma}\right)$ is our measurable space of states.

\subsection{Domain of measurable expectations}

As the weakest preexpectation semantics of PL is defined in terms of an operator transforming measurable functions, we need to show that measurable functions from $\Omega_{\sigma}$ to $\overline{\mathbb{R}}_{+}=\mathbb{R}_{+} \cup\{+\infty\}$ form a valid domain. More specifically, these functions must form a $\omega$-complete partial order (whose definition is included in Appendix B). Similarly, we need to show that the domain of bounded measurable expectations, which will be used in the weakest liberal preexpectation semantics, is valid. Fortunately, these facts follow immediately from basic properties of measure theory.

Lemma 2. The set of measurable functions $f: \Omega_{\sigma} \rightarrow \overline{\mathbb{R}}_{+}$with point-wise ordering forms an $\omega$-complete partial order ( $\omega$-cpo). Similarly, the set of bounded measurable functions $f: \Omega_{\sigma} \rightarrow[0,1]$ forms an $\omega$-cpo.

Proof. The bottom element of the set of measurable functions $f: \Omega_{\sigma} \rightarrow \overline{\mathbb{R}}_{+}$is the function $\lambda \sigma .0$, mapping every state to 0 . It is known that any increasing chain of functions with co-domain $\overline{\mathbb{R}}_{+}$has a supremum, so this also holds for chains of measurable functions. The fact that point-wise supremum of measurable functions to $\overline{\mathbb{R}}_{+}$is measurable is a standard result in measure theory. The argument for bounded measurable functions is the same.

\subsection{Expression and predicate evaluation}

The semantics makes use of two evaluation functions, $\sigma(E)$ and $\sigma(\phi)$, which evaluate the real-valued expression $E$ and predicate $\phi$, respectively, in state $\sigma$. We assume that for each $E$, the evaluation function on states $\lambda(\sigma, E) \cdot \sigma(E)$ is measurable and, similarly, for all $\phi$, the function $\lambda(\sigma, \phi) \cdot \sigma(\phi)$ is measurable 3 . We also assume that the evaluation functions are total - this means that in case of evaluation errors, such as some variable in $E$ not being in the domain of $\sigma$, some value (typically 0 or false) still needs to be returned. We convert truth values to reals by Iverson brackets [.]: [true $]=1$ and [false] $=0$. We write $E$ for $\lambda \sigma . \sigma(E)$ and $\phi$ for $\lambda \sigma . \sigma(\phi)$ if it is clear from the context that $E$ or $\phi$ denotes a function.

${ }^{3}$ This assumption requires a $\sigma$-algebra on expressions and predicates. This can be defined as a Borel $\sigma$-algebra induced by a simple metric on syntactic terms, as in [ $[5]$. 


$$
\begin{aligned}
& \operatorname{wp} \llbracket \operatorname{skip} \rrbracket(f)=f \\
& \text { wp } \llbracket \text { diverge } \rrbracket(f)=0 \\
& \operatorname{wp} \llbracket x:=E \rrbracket(f)=\lambda \sigma \cdot f(\sigma[x \mapsto \sigma(E)]) \\
& \operatorname{wp} \llbracket x: \approx U \rrbracket(f)=\lambda \sigma \cdot \int_{[0,1]} f(\sigma[x \mapsto v]) \lambda(d v)
\end{aligned}
$$

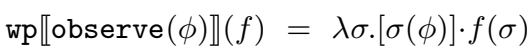

$$
\begin{aligned}
& \mathrm{wp} \llbracket \operatorname{score}(E) \rrbracket(f)=\lambda \sigma \cdot[\sigma(E) \in(0,1]] \cdot \sigma(E) \cdot f(\sigma) \\
& \operatorname{wp} \llbracket C_{1} ; C_{2} \rrbracket(f)=\operatorname{wp} \llbracket C_{1} \rrbracket\left(\operatorname{wp} \llbracket C_{2} \rrbracket(f)\right) \\
& \operatorname{wp} \llbracket \operatorname{if}(\phi)\{C\} \rrbracket(f)=[\phi] \cdot \operatorname{wp} \llbracket C \rrbracket(f)+[\neg \phi] \cdot f \\
& \operatorname{wp} \llbracket \operatorname{while}(\phi)\{C\} \rrbracket(f)=\operatorname{lfp} X .[\neg \phi] \cdot f+[\phi] \cdot \operatorname{wp} \llbracket C \rrbracket(X)
\end{aligned}
$$

Fig. 2. Weakest preexpectation semantics of PL

\subsection{Weakest preexpectation semantics}

We now have all ingredients in place to define the weakest preexpectation semantics of PL. This semantics is defined by the operator wp $\llbracket C \rrbracket(\cdot)$, which takes a measurable function $f$ from $\Omega_{\sigma}$ to $\overline{\mathbb{R}}_{+}$- called the postexpectation - and returns a measurable function in the same domain - called the preexpectation) - which, for every initial state $\sigma_{0}$, computes the expected value of $f$ after executing the program $C$ starting in state $\sigma_{0}$. In other words, if $f: \Omega_{\sigma} \rightarrow \overline{\mathbb{R}}_{+}$is a measurable function on states and $\sigma_{0} \in \Omega_{\sigma}$ is the initial state, then wp $\llbracket C \rrbracket(f)\left(\sigma_{0}\right)$ yields the expected value of $f(\sigma)$, where $\sigma$ is a final program state of $C$.

The wp-semantics of PL is defined by structural induction and is shown in Fig. 2. The semantics of most constructs matches the wp-semantics in 29], with the distinction that it is defined on the domain of nonnegative measurable functions on states, rather than arbitrary nonnegative functions. Let us briefly explain the individual cases one by one.

Skip. The skip statement leaves the expectation $f$ unchanged.

Divergence. The expectation of any function $f$ with respect to the diverge expression is 0 , as no final state at which $f$ can be evaluated is ever reached by the program.

Assignment. For assignment $x:=E$, the semantics just evaluates $E$, updates $x$ with the new value in the state and passes this updated state to the expectation.

Random draw. The expected value of a measurable function $f$ on states with respect to the uniform random assignment $x: \approx U$, applied to the initial state $\sigma$, is the Lebesgue integral of $f(\sigma[x \mapsto v])$ (as a function of $v$ ) with 
respect to the Lebesgue measure $\mu_{L}$ on $[0,1]$. By the Fubini-Tonelli theorem, $\operatorname{wp} \llbracket x: \approx U \rrbracket(f)$ is itself a measurable function.

Hard. The observe statement defines hard conditioning - it states that all runs of the program which do not satisfy $\phi$ should be discarded and should not affect the expectation of $f$.

Soft. Scoring multiplies the expectation by the argument to score, expected to evaluate to a number in $(0,1]$.

Sequencing. The semantics of a sequence $C_{1} ; C_{2}$ of two commands is just the composition of the semantics of respective commands - the semantics of $C_{2}$ with respect to the given input function $f$ is the input to the semantics of $C_{1}$.

Conditional. The semantics of an if $(\phi)\{C\}$-expression is, for initial states satisfying the condition $\phi$, the semantics of the body $C$. For other states, the semantics is equivalent to the skip statement, as the expression does not do anything.

Loops. The semantics of a while-loop is defined as the least fixpoint of a function which simply returns the input continuation $f$ if $\phi$ is false (corresponding to exiting the loop) and applies the semantics of the body to the argument $X$ otherwise (which corresponds to performing another iteration). As explained in the following paragraph (cf. Lemma 4), this has the desired effect that the semantics of a while-loop is equivalent to the semantics of the infinite unfolding of the loop.

Well-definedness and key properties of wp. When defining the weakest preexpectation semantics of PL, we implicitly assumed that all mathematical objects used are well defined. Specifically, we assumed that the wp transformer preserves measurability and that the least fixpoint in the semantics of while-loops exists. These properties can be proven by structural induction on the program $C$. The key observations used in the proof (which would not be needed in the discrete case) are that $\lambda \sigma \cdot \int_{[0,1]}\left(\sup _{i} f_{i}\right)(\sigma[x \mapsto v]) \mu_{L}(d v)=\lambda \sigma \sup _{i} \int_{[0,1]} f_{i}(\sigma[x \mapsto v]) \mu_{L}(d v)$ by Beppo Levi's theorem and that $\lambda \sigma \cdot \int_{[0,1]} f(\sigma[x \mapsto v]) \mu_{L}(d v)$ is measurable (as a function of $\sigma$ ) by the Fubini-Tonelli theorem. As both $\omega$-continuity (as defined in Appendix (B) and measurability are required for wp $\llbracket C \rrbracket$ to be well defined, we need to prove both properties simultaneously, so that the induction hypothesis is strong enough.

Lemma 3. For every program $C$ :

1. the function $\mathrm{wp} \llbracket C \rrbracket(\cdot)$ is $\omega$-continuous, and

2. for every measurable $f: \Omega_{\sigma} \rightarrow \overline{\mathbb{R}}_{+}$, wp $\llbracket C \rrbracket(f)(\cdot)$ is measurable.

Proof. By induction on the structure of $C$.

The continuity of wp $\llbracket C \rrbracket$ also ensures that the expression $[\neg \phi] \cdot f+[\phi] \cdot \operatorname{wp} \llbracket C \rrbracket(X)$ in the semantics of while loops is continuous as a function of $X$. Applying Kleene's Fixpoint Theorem immediately gives us the following result:

4 The Lebesgue measure is usually denoted by $\lambda$ in the literature. We write $\mu_{L}$ instead to avoid confusion with the use of $\lambda \sigma$ to define a function with formal parameter $\sigma$. 
Lemma 4. Let $f: \Omega_{\sigma} \rightarrow \overline{\mathbb{R}}_{+}$be measurable, $C$ be a PL program and $\phi$ be a predicate. Let ${ }_{\langle\phi, C\rangle}^{\mathrm{wp}} \Phi_{f}(X)=[\neg \phi] \cdot f+[\phi] \cdot \mathrm{wp} \llbracket C \rrbracket(X)$. Then $\operatorname{lfp} X \cdot{ }_{\langle\phi, C\rangle}^{\mathrm{wp}} \Phi_{f}(X)$ exists and is equal to $\sup _{n}{ }_{\langle\phi, C\rangle} \Phi_{f}^{n}(0)$. Thus, wp【while $(\phi)\{C\} \rrbracket(f)$ exists and

$$
\operatorname{wp} \llbracket \operatorname{while}(\phi)\{C\} \rrbracket(f)=\sup _{n} \operatorname{wp}_{\langle\phi, C\rangle} \Phi_{f}^{n}(0)
$$

\subsection{Examples}

Having defined the weakest preexpectation semantics, we explain it using a few examples. We first introduce two simple examples, which illustrate the key concepts, and then show how the semantics can be applied to the tortoise and hare program from the introduction.

Notation. To distinguish between program variables and metavariables, we write the former in fixed-width font (such as $\mathrm{x} 1$ ) and the latter in the usual italic form (such as $x_{1}$ ). In functions where only the original, non-updated input state appears in the body, we sometimes make the state implicit by removing " $\lambda \sigma$." and replacing variable lookups of the form $\sigma(\mathrm{x})$ by just variables. For instance we write $\lambda \sigma \cdot \sigma(\mathrm{x})+\sigma(\mathrm{y})$ simply as $\mathrm{x}+\mathrm{y}$.

Example 1. Let us first consider a very simple instance of Bayesian linear regression. We want to fit a linear function approximately to two points $(0,2)$ and $(1,3)$, assuming that the coefficients of the function have Gaussian prior distributions. A PL implementation of such a regression, using the sof teq distance squashing function mentioned at the end of Section 2, has the following form:

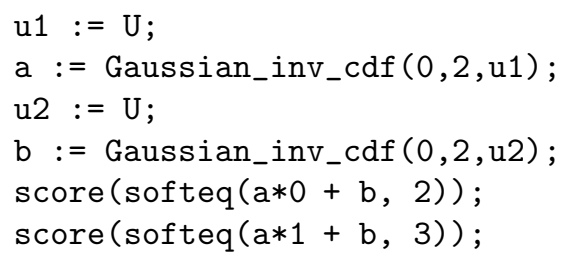

Let us now suppose that we want to calculate the expected value of the square of thea coefficient (recall that we can only compute expectations of nonnegative functions). We can do that by computing the weakest preexpectation of $\lambda \sigma \cdot \sigma(\mathrm{a})^{2}$ (written $\mathrm{a}^{2}$ in short) with respect to the above program - that is, wp $\llbracket C \rrbracket\left(\mathrm{a}^{2}\right)$, where $C$ is the given program. In the following derivation (as well as subsequent examples in this chapter), we adopt the notation used in 21], where the function directly below a statement $C$ is a postexpectation, and the function directly above $C$ is the corresponding preexpectation. That is, a block of the form:

$$
\begin{array}{r}
/ / f_{1} \\
C \\
/ / f_{2}
\end{array}
$$


states that $f_{1}=\operatorname{wp} \llbracket C \rrbracket\left(f_{2}\right)$. We also use the letter $\mathrm{G}$ as an abbreviation for the Gaussian inverse cdf 5 .

We can derive the expected value of $\mathrm{a}^{2}$ as shown below. Note that the expressions between program lines are functions on program states, written using the implicit notation explained before.

$$
\begin{aligned}
& / / \int_{(0,1)} \int_{(0,1)} e^{-\left(\mathrm{G}\left(0,2, v_{2}\right)-2\right)^{2}-\left(\mathrm{G}\left(0,2, v_{1}\right)+\mathrm{G}\left(0,2, v_{2}\right)-3\right)^{2}} \cdot \mathrm{G}\left(0,2, v_{1}\right)^{2} \mu_{L}\left(d v_{2}\right) \mu_{L}\left(d v_{1}\right) \\
& \mathrm{u} 1:=\mathrm{U} ; \\
& / / \int_{(0,1)} e^{-\left(\mathrm{G}\left(0,2, v_{2}\right)-2\right)^{2}-\left(\mathrm{G}(0,2, \mathrm{u} 1)+\mathrm{G}\left(0,2, v_{2}\right)-3\right)^{2}} \cdot \mathrm{G}(0,2, \mathrm{u} 1)^{2} \mu_{L}\left(d v_{2}\right) \\
& \mathrm{a}:=\text { Gaussian_inv_cdf }(0,2, \mathrm{u} 1) ; \\
& / / \int_{(0,1)} e^{-\left(\mathrm{G}\left(0,2, v_{2}\right)-2\right)^{2}-\left(\mathrm{a}+\mathrm{G}\left(0,2, v_{2}\right)-3\right)^{2}} \cdot \mathrm{a}^{2} \mu_{L}\left(d v_{2}\right) \\
& \quad \mathrm{u} 2:=\mathrm{U} ; \\
& / / e^{-(\mathrm{G}(0,2, \mathrm{u} 2)-2)^{2}-(\mathrm{a}+\mathrm{G}(0,2, \mathrm{u} 2)-3)^{2} \cdot \mathrm{a}^{2}} \\
& \quad \mathrm{~b}:=\mathrm{Gaussian}{ }^{\mathrm{inn}}-\mathrm{cdf}(0,2, \mathrm{u} 2) ; \\
& / / e^{-(\mathrm{b}-2)^{2}-(\mathrm{a}+\mathrm{b}-3)^{2}} \cdot \mathrm{a}^{2} \\
& \quad \text { score }(\text { softeq }(\mathrm{a} * 0+\mathrm{b}, 2)) ; \\
& / / e^{-(\mathrm{a}+\mathrm{b}-3)^{2} \cdot \mathrm{a}^{2}} \\
& \quad \text { score }(\text { softeq }(\mathrm{a} * 1+\mathrm{b}, 3)) ; \\
& / / \mathrm{a}^{2}
\end{aligned}
$$

We observe that the expected value of $\mathrm{a}^{2}$ is independent on the initial state, which is not surprising as the program has no free variables. For any initial state $\sigma$, the expected value wp $\llbracket C \rrbracket\left(\mathrm{a}^{2}\right)(\sigma)$ of a is:

$$
\int_{(0,1)} \int_{(0,1)} e^{-\left(\mathrm{G}\left(0,2, v_{2}\right)-2\right)^{2}-\left(\mathrm{G}\left(0,2, v_{1}\right)+\mathrm{G}\left(0,2, v_{2}\right)-3\right)^{2}} \cdot \mathrm{G}\left(0,2, v_{1}\right)^{2} \mu_{L}\left(d v_{2}\right) \mu_{L}\left(d v_{1}\right) .
$$

We can also represent this expression as a double integral with respect to the Gaussian probability distribution $\mathcal{D}_{\mathrm{G}}$ with mean 0 and variance 2 , using the fact that a continuous probability distribution is a pushforward of the Lebesgue measure by the inverse cdf of the given distribution:

$$
\operatorname{wp} \llbracket C \rrbracket(\mathrm{a})(\sigma)=\iint e^{-\left(x_{2}-2\right)^{2}-\left(x_{1}+x_{2}-3\right)^{2}} \cdot x_{1}^{2} \mathcal{D}_{\mathrm{G}}\left(d x_{2}\right) \mathcal{D}_{\mathrm{G}}\left(d x_{1}\right) .
$$

This expression can also be represented as a double integral of Gaussian densities (denoted $G_{p d f}$ ) over $\mathbb{R}$ :

${ }^{5}$ We can integrate the Gaussian inverse over the interval $(0,1)$ instead of $[0,1]$, because the value of the Lebesgue integral at a single point does not contribute to the result 


$$
\iint e^{-\left(x_{2}-2\right)^{2}-\left(x_{1}+x_{2}-3\right)^{2}} \mathbf{G}_{p d f}\left(0,2, x_{1}\right) \mathbf{G}_{p d f}\left(0,2, x_{2}\right) \cdot x_{1}^{2} \mu_{L}\left(d x_{2}\right) \mu_{L}\left(d x_{1}\right) .
$$

Example 2. Let us now consider a very simple example of a potentially diverging program with continuous variables and soft conditioning. This example may be rather contrived and does not represent any machine learning model, but it illustrates well how the semantics works. Take the following program $C$ :

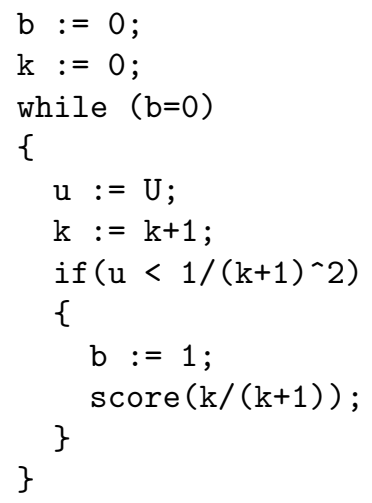

Suppose we want to compute wp $\llbracket C \rrbracket(1)$, that is, the weakest preexpectation of the constant function $\lambda \sigma .1$ with respect to the program $C$. This may be interpreted as the probability that the program terminates, weighted by the scores.

As the program has a while-loop, we need to find the characteristic function ${ }_{\left\langle b=0, C^{\prime}\right\rangle} \Phi_{1}$ of this loop (whose body we denote by $C^{\prime}$ ), with respect to the constant postexpectation 1 . In this case, the characteristic function is

$$
{ }_{\left\langle b=0, C^{\prime}\right\rangle} \Phi_{1}(X)=[\mathrm{b} \neq 0]+[\mathrm{b}=0] \cdot \operatorname{wp} \llbracket C^{\prime} \rrbracket(X) .
$$


We first need to compute wp $\llbracket C^{\prime} \rrbracket(X)$ :

$$
\begin{aligned}
& / / \lambda \sigma \cdot \frac{\sigma(\mathrm{k})+1}{(\sigma(\mathrm{k})+2)^{3}} \cdot X(\sigma[k \mapsto \sigma(k)+1][\mathrm{b} \mapsto 1])+\frac{(\sigma(\mathrm{k})+2)^{2}-1}{(\sigma(\mathrm{k})+2)^{2}} \cdot X(\sigma[k \mapsto \sigma(k)+1]) \\
& = \\
& / / \lambda \sigma \cdot \int_{[0,1]}\left[v<\frac{1}{(\sigma(\mathrm{k})+2)^{2}}\right] \cdot \frac{\sigma(\mathrm{k})+1}{\sigma(\mathrm{k})+2} \cdot X(\sigma[k \mapsto \sigma(k)+1][\mathrm{b} \mapsto 1]) \\
& / / \quad+\left[v \geq \frac{1}{(\sigma(\mathrm{k})+2)^{2}}\right] \cdot X(\sigma[k \mapsto \sigma(k)+1]) \mu_{L}(d v) \\
& \mathrm{u}:=\mathrm{U} \text {; } \\
& / / \lambda \sigma \cdot\left[\sigma(\mathrm{u})<\frac{1}{(\sigma(\mathrm{k})+2)^{2}}\right] \cdot \frac{\sigma(\mathrm{k})+1}{\sigma(\mathrm{k})+2} \cdot X(\sigma[k \mapsto \sigma(k)+1][\mathrm{b} \mapsto 1]) \\
& \mathrm{k}:=\mathrm{k}+1 \\
& +\left[\sigma(\mathrm{u}) \geq \frac{1}{(\sigma(\mathrm{k})+2)^{2}}\right] \cdot X(\sigma[k \mapsto \sigma(k)+1]) \\
& / / \lambda \sigma \cdot\left[\sigma(\mathrm{u})<\frac{1}{(\sigma(\mathrm{k})+1)^{2}}\right] \cdot \frac{\sigma(\mathrm{k})}{\sigma(\mathrm{k})+1} \cdot X(\sigma[\mathrm{b} \mapsto 1])+\left[\sigma(\mathrm{u}) \geq \frac{1}{(\sigma(\mathrm{k})+1)^{2}}\right] \cdot X(\sigma) \\
& \text { if }\left(\mathrm{u}<1 /(\mathrm{k}+1)^{2}\right) \\
& \{ \\
& / / \lambda \sigma \cdot \frac{\sigma(\mathrm{k})}{\sigma(\mathrm{k})+1} \cdot X(\sigma[\mathrm{b} \mapsto 1]) \\
& \mathrm{b}:=1 \text {; } \\
& \text { // } \lambda \sigma \cdot \frac{\sigma(\mathrm{k})}{\sigma(\mathrm{k})+1} \cdot X(\sigma) \\
& \operatorname{score}(\mathrm{k} /(\mathrm{k}+1)) \text {; } \\
& \text { // X } \\
& \text { \} } \\
& \text { // X }
\end{aligned}
$$

To simplify the presentation, we assumed in the last step that $X$ does not depend directly on the variable $\mathrm{u}$ - we can show by a simple induction that this holds for $X={ }_{\left\langle b=0, C^{\prime}\right\rangle} \Phi_{1}^{n}(0)$ for any $n$, and we only need to apply wp $\llbracket C^{\prime} \rrbracket(\cdot)$ to functions $X$ of this form. By plugging

$$
\begin{aligned}
\operatorname{wp} \llbracket C^{\prime} \rrbracket(X)= & \lambda \sigma \cdot \frac{\sigma(\mathrm{k})+1}{(\sigma(\mathrm{k})+2)^{3}} \cdot X(\sigma[k \mapsto \sigma(k)+1][\mathrm{b} \mapsto 1]) \\
& +\frac{(\sigma(\mathrm{k})+2)^{2}-1}{(\sigma(\mathrm{k})+2)^{2}} \cdot X(\sigma[k \mapsto \sigma(k)+1])
\end{aligned}
$$

into the equation for the characteristic function, we get

$$
\begin{aligned}
& \operatorname{wp}_{\left\langle b=0, C^{\prime}\right\rangle} \Phi_{1}(X)=\lambda \sigma \cdot[\sigma(b) \neq 0]+[\sigma(b)=0]\left(\frac{\sigma(\mathrm{k})+1}{(\sigma(\mathrm{k})+2)^{3}} X(\sigma[k \mapsto \sigma(k)+1][\mathrm{b} \mapsto 1])\right. \\
& \left.+\frac{(\sigma(\mathrm{k})+2)^{2}-1}{(\sigma(\mathrm{k})+2)^{2}} X(\sigma[k \mapsto \sigma(k)+1])\right)
\end{aligned}
$$


We can now calculate subsequent terms of the sequence $\underset{\left\langle b=0, C^{\prime}\right\rangle}{\mathrm{wp}} \Phi_{1}^{n}(0)$, whose supremum is the semantics of the while-loop:

$$
\begin{aligned}
& \operatorname{wp}_{\left\langle\mathrm{b}=0, C^{\prime}\right\rangle} \Phi_{1}^{0}(0)=0 \\
& \underset{\text { wp }}{\left\langle\mathrm{b}=0, C^{\prime}\right\rangle} \Phi_{1}^{1}(0)=[\mathrm{b} \neq 0] \\
& \operatorname{wp}_{\left\langle\mathrm{b}=0, C^{\prime}\right\rangle} \Phi_{1}^{2}(0)=[\mathrm{b} \neq 0]+[b=0] \frac{k+1}{k+2} \cdot \frac{1}{(k+2)^{2}} \\
& \operatorname{wp}_{\left\langle\mathrm{b}=0, C^{\prime}\right\rangle} \Phi_{1}^{3}(0)=[\mathrm{b} \neq 0]+[b=0] \frac{k+1}{k+2} \cdot\left(\frac{1}{(k+2)^{2}}+\frac{1}{(k+3)^{2}}\right) \\
& \operatorname{wp}_{\left\langle\mathrm{b}=0, C^{\prime}\right\rangle} \Phi_{1}^{4}(0)=[\mathrm{b} \neq 0]+[\mathrm{b}=0] \frac{k+1}{k+2} \cdot\left(\frac{1}{(k+2)^{2}}+\frac{1}{(k+3)^{2}}+\frac{1}{(k+4)^{2}}\right) \\
& \ldots
\end{aligned}
$$

It follows that $\underset{\left\langle b=0, C^{\prime}\right\rangle}{\mathrm{wp}} \Phi_{1}^{n}(0)$ can be represented in a closed form for any $n$ :

$$
\operatorname{wp}_{\left\langle b=0, C^{\prime}\right\rangle} \Phi_{1}^{n}(0)=[b \neq 0]+[b=0] \cdot \frac{k+1}{k+2} \cdot\left(\sum_{i=2}^{n} \frac{1}{(k+i)^{2}}\right)
$$

The correctness of this formula can be proven by a simple induction on $n$ (which we omit here). This means that the semantics of the while-loop has the form:

$$
\begin{aligned}
\operatorname{wp} \llbracket \text { while }(\mathrm{b}=0)\left\{C^{\prime}\right\} \rrbracket(1) & =\sup _{n} \mathrm{wp}_{\left\langle b=0, C^{\prime}\right\rangle} \Phi_{1}^{n}(0) \\
& =[b \neq 0]+[b=0] \frac{k+1}{k+2} \cdot\left(\sum_{i=2}^{\infty} \frac{1}{(k+i)^{2}}\right)
\end{aligned}
$$

We can now use this result to compute the postexpectation of $\sigma .1$ with respect to the full program (where the while-loop is the program $C^{\prime}$, whose semantics has already been calculated):

$$
\begin{aligned}
& / / \frac{\pi^{2}}{12}-\frac{1}{2} \\
& =\frac{1}{2} \cdot \sum_{i=2}^{\infty} \frac{1}{i^{2}} \\
& \mathrm{~b}:=0 ; \\
& / / \quad[\mathrm{b} \neq 0]+[\mathrm{b}=0] \frac{1}{2} \cdot\left(\sum_{i=2}^{\infty} \frac{1}{i^{2}}\right) \\
& \quad \mathrm{k}=0 ; \\
& / /[\mathrm{b} \neq 0]+[b=0] \frac{\mathrm{k}+1}{\mathrm{k}+2} \cdot\left(\sum_{i=2}^{\infty} \frac{1}{(\mathrm{k}+i)^{2}}\right) \\
& \quad \mathrm{C}^{\prime} \\
& 1
\end{aligned}
$$


In the last step, we used the well-known fact that the series $\sum_{i=2}^{\infty} \frac{1}{i^{2}}$ converges to $\frac{\pi^{2}}{6}-1$, to establish that $\operatorname{wp} \llbracket C \rrbracket(1)=\frac{\pi^{2}}{12}-\frac{1}{2}$.

Example 3. In order to illustrate the wp semantics on a more realistic program, let us recall the tortoise and hare example with soft conditioning from the introduction (with the time variable removed for simplicity and the Gaussian density in score replaced by softeq to ensure that scores are bounded). After expanding the syntactic sugar, this program has the following form:

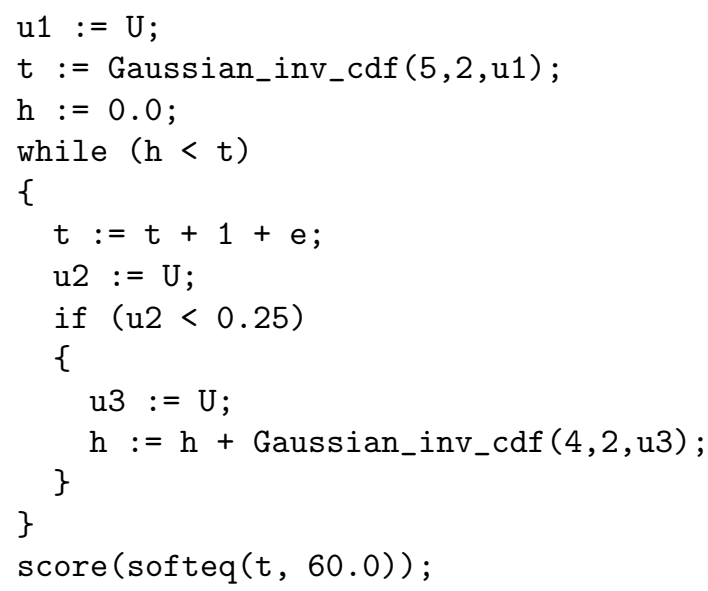

Let us suppose we want to calculate the expected distance travelled by the tortoise before it gets caught. To this end, we need to calculate wp $\llbracket C \rrbracket(t)$ for the above program $C$ and apply it to the empty initial state (or in fact any initial state, as the program contains no free variables).

Like in the previous example, we begin by calculating the characteristic function of the while-loop. We first need to compute wp $\llbracket C^{\prime} \rrbracket(X)$ for the loop body $C^{\prime}$. We assume that $X$ does not depend directly on variables u2 and u3 - this is safe for $X$ of the form $X={ }_{\left\langle h<t, C^{\prime}\right\rangle}^{\text {wp }} \Phi_{f}^{n}(0)$, as long as $f$ does not depend directly on the aforementioned variables. 


$$
\begin{aligned}
& / / \lambda \sigma \cdot 0.25 \cdot \int_{(0,1)} X(\sigma[\mathrm{t} \mapsto \sigma(\mathrm{t})+1+e][\mathrm{h} \mapsto \sigma(\mathrm{h}) \\
& \left.\left.+\mathrm{G}\left(4,2, v_{3}\right)\right]\right) \mu_{L}\left(d v_{3}\right)+0.75 \cdot X(\sigma[\mathrm{t} \mapsto \sigma(\mathrm{t})+1+e]) \\
& \mathrm{t}:=\mathrm{t}+1+\mathrm{e} \\
& / / \lambda \sigma .0 .25 \cdot \int_{(0,1)} X\left(\sigma\left[\mathrm{h} \mapsto \sigma(\mathrm{h})+\mathrm{G}\left(4,2, v_{3}\right)\right]\right) \mu_{L}\left(d v_{3}\right)+0.75 \cdot X(\sigma) \\
& = \\
& / / \lambda \sigma \cdot \int_{(0,1)}\left[v_{2}<0.25\right] \int_{(0,1)} X\left(\sigma\left[\mathrm{u} 2 \mapsto v_{2}\right]\left[\mathrm{u} 3 \mapsto v_{3}\right][\mathrm{h} \mapsto \sigma(\mathrm{h})\right. \\
& \left.\left.+\mathrm{G}\left(4,2, v_{3}\right)\right]\right) \mu_{L}\left(d v_{3}\right)+\left[v_{2} \geq 0.25\right] X\left(\sigma\left[\mathrm{u} 2 \mapsto v_{2}\right]\right) \mu_{L}\left(d v_{2}\right) \\
& \mathrm{u} 2:=\mathrm{U} \\
& / / \lambda \sigma \cdot[\sigma(\mathrm{u} 2)<0.25] \int_{(0,1)} X\left(\sigma\left[\mathrm{u} 3 \mapsto v_{3}\right]\left[\mathrm{h} \mapsto \sigma(\mathrm{h})+\mathrm{G}\left(4,2, v_{3}\right)\right] \mu_{L}\left(d v_{3}\right)\right. \\
& +[\sigma(\mathrm{u} 2) \geq 0.25] \cdot X(\sigma) \\
& \text { if }(\mathrm{u} 2<0.25) \\
& \{ \\
& / / \lambda \sigma \cdot \int_{(0,1)} X\left(\sigma\left[\mathrm{u} 3 \mapsto v_{3}\right]\left[\mathrm{h} \mapsto \sigma(\mathrm{h})+\mathrm{G}\left(4,2, v_{3}\right)\right] \mu_{L}\left(d v_{3}\right)\right. \\
& \text { u3 }:=\mathrm{U} \text {; } \\
& / / \lambda \sigma \cdot X(\sigma[\mathrm{h} \mapsto \sigma(\mathrm{h})+\mathrm{G}(4,2, \sigma(\mathrm{u} 3))]) \\
& \mathrm{h}:=\mathrm{h}+\text { Gaussian_inv_cdf }(4,2, \mathrm{u} 3) \text {; } \\
& \text { // } X \\
& \text { \} } \\
& \text { // X }
\end{aligned}
$$

Thus, we have ${ }_{\left\langle h<t, C^{\prime}\right\rangle} \Phi_{f}(X)=\lambda \sigma .[\sigma(\mathrm{h}) \geq \sigma(\mathrm{t})] \cdot f(\sigma)+[\sigma(\mathrm{h})<\sigma(\mathrm{t})] \cdot(0.25$. $\left.\left.\int_{[0,1]} X\left(\sigma_{\sigma, v_{3}}^{\prime}\right) \mu_{L}\left(d v_{3}\right)+0.75 \cdot X\left(\sigma_{\sigma}^{\prime \prime}\right)\right)\right)$, where $\sigma_{\sigma, v_{3}}^{\prime}=\sigma[\mathrm{t} \mapsto \sigma(\mathrm{t})+1+e][h \mapsto$ $\left.\sigma(\mathrm{h})+\mathrm{G}\left(4,2, v_{3}\right)\right]$ is the state $\sigma$ updated after a step where both the tortoise and the hare moved (the latter by $\mathrm{G}\left(4,2, v_{3}\right)$ ) and $\sigma_{\sigma}^{\prime \prime}=\sigma[\mathrm{t} \mapsto \sigma(\mathrm{t})+1+e]$ is state $\sigma$ updated after a step where the hare stood still.

By the inductive definition of the wp operator, we have wp $\llbracket$ while $(\mathrm{h}<\mathrm{t})\left\{C^{\prime}\right\} \rrbracket=$ $\sup _{n} \underset{\text { wp }}{\left\langle h<t, C^{\prime}\right\rangle} \Phi_{f}^{n}(0)$. Then $\sup _{n} \stackrel{\text { wp }}{\left\langle h<t, C^{\prime}\right\rangle} \Phi_{f}^{n}(0)$ is guaranteed to exist, but unlike in the previous example, it does not have a nice closed form. This is indeed the case for most real-world programs.

We can now derive the formula for the expected final value of $t$ : 


$$
\begin{aligned}
& / / \lambda \sigma \cdot \int_{(0,1)} \sup _{n} \operatorname{wp}_{\left\langle h<t, C^{\prime}\right\rangle} \Phi_{e^{-(\mathrm{t}-60.0)^{2} \mathrm{t}}}^{n}(0) \\
& / / \quad\left(\sigma\left[t \mapsto \mathrm{G}\left(5,2, \sigma\left(v_{1}\right)\right)\right][\mathrm{h} \mapsto 0]\right) \mu_{L}\left(d v_{1}\right) \\
& = \\
& / / \lambda \sigma \cdot \int_{(0,1)} \sup _{n} \operatorname{wp}_{\left\langle h<t, C^{\prime}\right\rangle} \Phi_{e^{-(\mathrm{t}-60.0)^{2} \mathrm{t}}}^{n}(0) \\
& \left(\sigma\left[\mathrm{u} 1 \mapsto v_{1}\right][t \mapsto \mathrm{G}(5,2, \sigma(\mathrm{u} 1))][\mathrm{h} \mapsto 0]\right) \mu_{L}\left(d v_{1}\right) \\
& \mathrm{u} 1:=\mathrm{U} \\
& / / \lambda \sigma \cdot \sup _{n}{ }_{\left\langle h<t, C^{\prime}\right\rangle} \Phi_{e^{-(\mathrm{t}-60.0)^{2} \mathrm{t}}}^{n}(0)(\sigma[t \mapsto \mathrm{G}(5,2, \sigma(\mathrm{u} 1))][\mathrm{h} \mapsto 0]) \\
& \mathrm{t}:=\text { Gaussian_inv_cdf }(5,2, \mathrm{u} 1) \text {; } \\
& / / \lambda \sigma \cdot \sup _{n}{ }_{\left\langle h<t, C^{\prime}\right\rangle}^{\mathrm{WP}} \Phi_{e^{-(\mathrm{t}-60.0)^{2} \mathrm{t}}}^{n}(0)(\sigma[\mathrm{h} \mapsto 0]) \\
& \mathrm{h}:=0.0 \text {; } \\
& / / \sup _{n} \operatorname{wp}_{\left\langle h<t, C^{\prime}\right\rangle} \Phi_{e^{-(\mathrm{t}-60.0)^{2} \mathrm{t}}}^{n}(0) \\
& \text { while }(\mathrm{h}<\mathrm{t})\{\ldots\} \\
& / / e^{-(\mathrm{t}-60.0)^{2}} \mathrm{t} \\
& \text { score }(\operatorname{softeq}(\mathrm{t}, 60.0)) \text {; } \\
& / / \mathrm{t}
\end{aligned}
$$

In the last step, we used the fact that ${ }_{\left\langle h<t, C^{\prime}\right\rangle}^{\mathrm{wp}} \Phi_{\text {softeq(t,60.0)t }}^{n}(0)$ does not depend directly on $\mathrm{u} 1$. We have now derived the expression for the weakest preexpectation semantics of the program $C$ :

$$
\mathrm{wp} \llbracket \mathrm{C} \rrbracket(t)=\lambda \sigma \cdot \int_{(0,1)} \sup _{n} \Phi^{n}(0)\left(\sigma\left[t \mapsto \text { Gaussian_inv_cdf }\left(5,2, \sigma\left(v_{1}\right)\right)\right][\mathrm{h} \mapsto 0]\right)
$$

where

$$
\begin{aligned}
& \Phi(X)=\lambda \sigma \cdot[\sigma(\mathrm{h}) \geq \sigma(\mathrm{t})] \cdot \operatorname{softeq}(\sigma(\mathrm{t}), 60.0) \sigma(\mathrm{t}) \\
&+[\sigma(\mathrm{h})<\sigma(\mathrm{t})] \cdot\left(0.25 \cdot \int_{(0,1)} X\left(\sigma_{\sigma, v_{3}}^{\prime}\right) \mu_{L}\left(d v_{3}\right)\right. \\
&\left.\left.+0.75 \cdot X\left(\sigma_{\sigma}^{\prime \prime}\right)\right)\right) \\
& \sigma_{\sigma, v_{3}}^{\prime}=\sigma[\mathrm{t} \mapsto \sigma(\mathrm{t})+1+e]\left[h \mapsto \sigma(\mathrm{h})+\text { Gaussian_inv_cdf }\left(4,2, v_{3}\right)\right] \\
& \sigma_{\sigma}^{\prime \prime}=\sigma[\mathrm{t} \mapsto \sigma(\mathrm{t})+1+e] .
\end{aligned}
$$

\subsection{Weakest liberal preexpectation semantics}

We now define a different variant of the above semantics, called the weakest liberal preexpectation semantics (wlp). In standard, discrete pGCL without scores 25, 29], the weakest liberal preexpectation defines the expected value of a function bounded by 1 (as per wp) plus the probability of divergence - in other words, in 


$$
\begin{aligned}
& \operatorname{wlp} \llbracket \operatorname{skip} \rrbracket(f)=f \\
& \text { wlp } \llbracket \text { diverge } \rrbracket(f)=1 \\
& \mathrm{w} l \mathrm{p} \llbracket x:=E \rrbracket(f)=\lambda \sigma \cdot f(\sigma[x \mapsto \sigma(E)]) \\
& \operatorname{wlp} \llbracket x: \approx U \rrbracket(f)=\lambda \sigma \cdot \int_{[0,1]} f(\sigma[x \mapsto v]) \mu_{L}(d v) \\
& \text { wlp [observe }(\phi) \rrbracket(f)=\lambda \sigma \cdot[\sigma(\phi)] \cdot f(\sigma) \\
& \operatorname{wlp} \llbracket \operatorname{score}(E) \rrbracket(f)=\lambda \sigma \cdot[\sigma(E) \in(0,1]] \cdot \sigma(E) \cdot f(\sigma)
\end{aligned}
$$

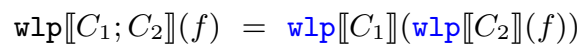

$$
\begin{aligned}
& \operatorname{wlp} \llbracket \operatorname{if}(\phi)\{C\} \rrbracket(f)=[\phi] \cdot \operatorname{wlp} \llbracket C \rrbracket(f)+[\neg \phi] \cdot f \\
& \text { wlp } \llbracket \operatorname{while}(\phi)\{C\} \rrbracket(f)=\operatorname{gfp} X \cdot[\neg \phi] \cdot f+[\phi] \cdot \operatorname{wlp} \llbracket C \rrbracket(X)
\end{aligned}
$$

Fig. 3. Weakest liberal preexpectation semantics of PL

contrast to wp, the wlp operator considers the value of the input function to be 1 , rather than 0 , for diverging program runs. If the input function is a binary predicate $\phi$, wlp defines the probability of this predicate being satisfied in the final state or the program never terminating.

In PL, the concept of weakest liberal preexpectation is similar, except that probabilities of all outcomes again have to be multiplied by scores encountered during the program's execution. Formally, wp $\llbracket C \rrbracket(\cdot)$, takes a measurable function $f$ mapping $\Omega_{\sigma}$ to $[0,1]$ and returns another measurable function from $\Omega_{\sigma}$ to $[0,1]$. Note that, in contrast to wp, the domain of the input function is restricted to the unit interval.

The wlp operator is defined in Fig. 3, with changes from wp marked in blue.

The semantics of a while loop is now computed with the greatest fixpoint rather than the least fixpoint - this has the effect that the "default" outcome for diverging loops is 1 instead of 0 . Similarly, diverge converts every function into a constant 1 function. The remaining changes are just that the recursive invocations to wp are replaced with calls to wlp.

Well-definedness of wlp. To show that the liberal semantics is well-defined, we use a similar argument as for wp. First, note that if we restrict the set of measurable functions $f: \Omega_{\sigma} \rightarrow \overline{\mathbb{R}}_{+}$to functions $f: \Omega_{\sigma} \rightarrow[0,1]$ with values in $[0,1]$, the constant function $\lambda \sigma .1$ (denoted 1 in short) is its top element. Hence, we can invert the complete partial order to get an $\omega$-cpo with inverse pointwise ordering and a "bottom" element 1 . The supremum of functions in this inverted cpo corresponds to the infimum in the original cpo, so continuity of wlp can again be proven by induction using the same domain-theoretic results. In the proof of measurability of wlp $\llbracket C \rrbracket(f)$, we use the fact that the infimum of a sequence of measurable functions is measurable, just like with supremum. By Kleene's 
Fixpoint Theorem, we again know that gfp $X .[\neg \phi] \cdot f+[\phi] \cdot w l p \llbracket C \rrbracket(X)$ exists and equals $\inf _{n} \underset{\langle\phi, C\rangle}{\mathrm{wlp}} \Phi_{f}^{n}(1)$, where ${ }_{\langle\phi, C\rangle}^{\mathrm{wlp}} \Phi_{f}(X)=[\neg \phi] \cdot f+[\phi] \cdot \mathrm{wl} \mathrm{p} \llbracket C \rrbracket(X)$.

Note that the weakest liberal preexpectation is only defined for bounded postexpectations $f$, as we need some upper bound to set the preexpectation to in case of divergence. If we chose this bound to be $\lambda \sigma . \infty$, wlp would effectively always be set to $\infty$ for all non almost-surely terminating programs, rendering the semantics useless.

Example 4. To show how wlp differs from wp, let us consider Example 2 again. This time, we want to compute wlp $\llbracket C \rrbracket(1)$, where $C$ is again the full program. Like before, we begin by computing the semantics of the loop. As the body $C^{\prime}$ of the loop is itself loop (and diverge)-free, we have wlp $\llbracket C^{\prime} \rrbracket(1)=$ $\mathrm{wp} \llbracket C^{\prime} \rrbracket(1)=\lambda \sigma \cdot \frac{\sigma(\mathrm{k})+1}{(\sigma(\mathrm{k})+2)^{3}} X(\sigma[\mathrm{k} \mapsto \sigma(\mathrm{k})+1][\mathrm{b} \mapsto 1])+\frac{(\sigma(\mathrm{k})+2)^{2}-1}{(\sigma(\mathrm{k})+2)^{2}} X(\sigma[k \mapsto \sigma(k)+$ $1]))$, which implies ${ }_{\left\langle\mathrm{b}=0, C^{\prime}\right\rangle}^{\mathrm{wlp}} \Phi_{1}(X)={ }_{\left\langle\mathrm{b}=0, C^{\prime}\right\rangle} \Phi_{1}(X)=\lambda \sigma \cdot[\sigma(\mathrm{b}) \neq 0]+[\sigma(\mathrm{b})=$ $\left.0]\left(\frac{\sigma(\mathrm{k})+1}{(\sigma(\mathrm{k})+2)^{3}} X(\sigma[\mathrm{k} \mapsto \sigma(\mathrm{k})+1][\mathrm{b} \mapsto 1])+\frac{(\sigma(\mathrm{k})+2)^{2}-1}{(\sigma(\mathrm{k})+2)^{2}} X(\sigma[k \mapsto \sigma(k)+1])\right)\right)$. The first terms of the sequence $\underset{\left\langle\mathrm{b}=0, C^{\prime}\right\rangle}{\mathrm{wlp}} \Phi_{1}(1)$ are as follows:

$$
\begin{aligned}
& { }_{\left\langle\mathrm{b}=0, C^{\prime}\right\rangle} \Phi_{1}^{0}(1)=[\mathrm{b} \neq 0] \\
& \operatorname{wlp}_{\left\langle\mathrm{b}=0, C^{\prime}\right\rangle} \Phi_{1}^{1}(1)=[\mathrm{b} \neq 0]+[\mathrm{b}=0]\left(\frac{\mathrm{k}+1}{\mathrm{k}+2} \frac{1}{(\mathrm{k}+2)^{2}}+\frac{(\mathrm{k}+2)^{2}-1}{(\mathrm{k}+2)^{2}}\right) \\
& \operatorname{wlp}_{\left\langle\mathrm{b}=0, C^{\prime}\right\rangle} \Phi_{1}^{2}(1)=[\mathrm{b} \neq 0]+[\mathrm{b}=0]\left(\frac{\mathrm{k}+1}{\mathrm{k}+2}\left(\frac{1}{(\mathrm{k}+2)^{2}}+\frac{1}{(\mathrm{k}+3)^{2}}\right)\right. \\
& \left.+\frac{(\mathrm{k}+2)^{2}-1}{(\mathrm{k}+2)^{2}} \cdot \frac{(\mathrm{k}+3)^{2}-1}{(\mathrm{k}+3)^{2}}\right) \\
& { }_{\left\langle\mathrm{b}=0, C^{\prime}\right\rangle} \Phi_{1}^{3}(1)=[\mathrm{b} \neq 0]+[\mathrm{b}=0]\left(\frac{\mathrm{k}+1}{\mathrm{k}+2}\left(\frac{1}{(\mathrm{k}+2)^{2}}+\frac{1}{(\mathrm{k}+3)^{2}}+\frac{1}{(\mathrm{k}+4)^{2}}\right)\right. \\
& \left.+\frac{(\mathrm{k}+2)^{2}-1}{(\mathrm{k}+2)^{2}} \cdot \frac{(\mathrm{k}+3)^{2}-1}{(\mathrm{k}+3)^{2}} \cdot \frac{(\mathrm{k}+4)^{2}-1}{(\mathrm{k}+4)^{2}}\right)
\end{aligned}
$$

We can now see what the pattern is:

$$
{ }_{\left\langle b=0, C^{\prime}\right\rangle} \Phi_{1}^{n}(0)=[b \neq 0]+[b=0] \cdot\left(\frac{k+1}{k+2} \cdot \sum_{i=2}^{n+1} \frac{1}{(k+i)^{2}}+\prod_{i=2}^{n+1} \frac{(\mathrm{k}+i)^{2}-1}{(\mathrm{k}+i)^{2}}\right)
$$

Moreover, we can quickly check that $\prod_{i=2}^{n+1} \frac{(\mathrm{k}+i)^{2}-1}{(\mathrm{k}+i)^{2}}=\frac{\mathrm{k}+1}{\mathrm{k}+2} \cdot \frac{\mathrm{k}+n+2}{\mathrm{k}+n+1}$. We obtain wlp $\llbracket C \rrbracket$ by computing the wlp of $\inf _{n} \underset{\left\langle b=0, C^{\prime}\right\rangle}{\text { wlp }} \Phi_{1}^{n}(0)$ with respect to the two initial statements, $\mathrm{k}:=0$ and $\mathrm{b}:=0$. Thus,

$$
\begin{aligned}
\mathrm{wl} \llbracket C \rrbracket(1) & =\frac{1}{2} \cdot \inf _{n} \sum_{i=2}^{n+1} \frac{1}{i^{2}}+\frac{1}{2} \frac{n+2}{n+1} \\
& =\frac{1}{2} \cdot \lim _{n \rightarrow \infty} \sum_{i=2}^{n+1} \frac{1}{i^{2}}+\frac{1}{2} \frac{n+2}{n+1}=\frac{1}{2} \cdot\left(\frac{\pi^{2}}{6}-1\right)+\frac{1}{2}=\frac{\pi^{2}}{12} .
\end{aligned}
$$




\subsection{Redundancy of score}

With respect to the weakest preexpectations semantics, the score operator admitting only arguments bounded by one is redundant, because scoring by a number in the unit interval can be simulated by rejection sampling without affecting the expected value of the given function. We show this result in this section.

To this end, we first need some additional concepts. Let $\operatorname{dom}(\sigma)$ be the set of variables which are assigned values in state $\sigma$. A function $f$ is said to be independent of a variable $x$ if $f(\sigma)=f(\sigma[x \mapsto V])$ for all $\sigma \in \Omega_{\sigma}$ and $V \in \mathbb{R}$. Let $\mathrm{f} v(E)$ be the set of free variables of an expression $E$, and $\operatorname{vars}(E)$ and $\operatorname{vars}(C)$ be the sets of all variables (free or bound) appearing in, respectively, the expression $E$ and the program $C$.

Lemma 5. For every expectation $f$, expression $E$ and variable $u$ such that $u \notin$ $\operatorname{vars}(E)$ and $f$ is independent of $u$, it holds:

$$
\begin{aligned}
\mathrm{wp} \llbracket \operatorname{score}(E) \rrbracket(f) & =\mathrm{wp} \llbracket u: \approx U ; \operatorname{observe}(E \in(0,1] \wedge u \leq E) \rrbracket(f) \\
\mathrm{wlp} \llbracket \operatorname{score}(E) \rrbracket(f) & =\operatorname{wlp} \llbracket u: \approx U ; \operatorname{observe}(E \in(0,1] \wedge u \leq E) \rrbracket(f) .
\end{aligned}
$$

Proof. For wp we have:

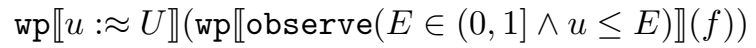

$$
\begin{aligned}
& =\lambda \sigma \cdot \int_{[0,1]} \text { wp } \llbracket \text { observe }(E \in(0,1] \wedge u \leq E) \rrbracket(f)(\sigma[u \mapsto v]) \mu_{L}(d v) \\
& =\lambda \sigma \cdot \int_{[0,1]}[\sigma[u \mapsto v](E) \in(0,1]][v \leq \sigma[u \mapsto v](E)] f(\sigma[u \mapsto v]) \mu_{L}(d v) \\
& { }_{(*)}=\lambda \sigma \cdot \int_{[0,1]}[\sigma(E) \in(0,1]][v \leq \sigma(E)] f(\sigma) \mu_{L}(d v) \\
& =\lambda \sigma \cdot f(\sigma) \int_{[0,1]}[\sigma(E) \in(0,1]][v \leq \sigma(E)] \mu_{L}(d v) \\
& \text { (Lebesgue) }=\lambda \sigma \cdot f(\sigma) \cdot \sigma(E) \\
& =\mathrm{wp} \llbracket \operatorname{score}(E) \rrbracket(f) .
\end{aligned}
$$

Proof step (*) follows from the fact that $u \notin \mathrm{fv}(E)$ and that $f$ is independent of $u$. The above result also proves the second item of the lemma, as wp and wlp coincide for programs without loops and diverge statements.

Let noscore $(C)$ denote the program obtained from program $C$ by replacing each expression of the form $\operatorname{score}(E)$ by $u: \approx U$; observe $(u \leq E)$ for sufficiently fresh variable $u \notin \operatorname{vars}(E)$. By "sufficiently fresh" we mean that $u$ does not appear in the program $C$ and that no function $f$ whose expected value we are interested in depends on $u$. (We do not formalise this notion for the sake of brevity.) 
Lemma 6. For every expectation $f$ we have:

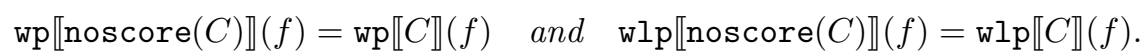

Proof. By induction on the structure of $C$, with appeal to Lemma 5 .

\section{Operational semantics}

In addition to the denotational semantics, we also present an operational semantics of PL. Apart from serving as a sanity check for the wp-semantics, this semantics is of interest on its own: an operational semantics is typically closer to a sample-based semantics that provides the basis for simulation-based evaluation of probabilistic programs (such as MCMC and Metropolis Hasting), is closer to models that are amenable to automated verification techniques such as probabilistic model checking 23], and sometimes simplifies for the reasoning about probabilistic programs, such as proving some sort of program equivalence 39].

\subsection{Entropy space}

A small-step operational semantics of a deterministic imperative language typically takes a program $C$ and state $\sigma$ and performs a single step of program evaluation, returning a new program $C^{\prime}$ and an updated state $\sigma^{\prime}$. For probabilistic languages, this is not possible, as a probabilistic program has multiple updated states - in fact, infinitely and uncountably many of them for programs with continuous distributions - depending on the outcomes of random draws. A possible way around this is to define the operational semantics of probabilistic languages with respect to a fixed sequence of values sampled from subsequent distributions, called a trace. By fixing a trace, a probabilistic program can be evaluated deterministically.

Traces often have the form of finite [5] or infinite 31] lists of values. To obtain a compositional semantics, we will instead use an abstract, infinite structure called entropy, as defined by [8] and [39].

Definition 1 ([39]). An entropy space is a measurable space $(\mathbb{S}, \mathcal{S})$ equipped with a measure $\mu_{\mathbb{S}}$ with $\mu_{\mathbb{S}}(\mathbb{S})=1$, and measurable functions $\pi_{U}: \mathbb{S} \rightarrow[0,1]$, $(::): \mathbb{S} \times \mathbb{S} \rightarrow \mathbb{S}, \pi_{L}, \pi_{R}: \mathbb{S} \rightarrow \mathbb{S}$ such that:

- For all measurable functions $f:[0,1] \rightarrow \overline{\mathbb{R}}_{+}$and Lebesgue measure $\lambda$,

$$
\int f\left(\pi_{U}(\theta)\right) \mu_{\mathbb{S}}(d \theta)=\int_{[0,1]} f(x) \mu_{L}(d x)
$$

- (::) is a surjective pairing function defined by: $\pi_{L}\left(\theta_{L}:: \theta_{R}\right)=\theta_{L}$ and $\pi_{R}\left(\theta_{L}::\right.$ $\left.\theta_{R}\right)=\theta_{R}$

- For all measurable functions $g: \mathbb{S} \times \mathbb{S} \rightarrow \overline{\mathbb{R}}_{+}$:

$$
\int g\left(\pi_{L}(\theta), \pi_{R}(\theta)\right) \mu_{\mathbb{S}}(d \theta)=\iint g\left(\theta_{L}, \theta_{R}\right) \mu_{\mathbb{S}}\left(d \theta_{L}\right) \mu_{\mathbb{S}}\left(d \theta_{R}\right) .
$$


An element $\theta \in \mathbb{S}$ of the entropy space is called an entropy.

In the above definition, $\mathbb{S}$ is the set of all possible entropies and $\mathcal{S}$ a $\sigma$-algebra on it. The entropy space is abstract, so we do not specify what $\mathbb{S}$ and $\mathcal{S}$ are and what they look like, we only assume that they satisfy the above properties.

Example of an entropy space A simple concrete realisation of the entropy space, for which the properties are satisfied, is the following:

- The set $\mathbb{S}$ is the set $[0,1]^{\omega}$ of infinite sequences of numbers in $[0,1]$ (the so-called Hilbert cube). Thus, each entropy $S \in \mathbb{S}$ is an infinite sequence $S=\left(s_{1}, s_{2}, s_{3} \ldots\right)$ such that $s_{i} \in[0,1]$ for all $i$.

- The $\sigma$-algebra $\mathcal{S}$ is, intuitively, the product of infinitely many copies of the Borel $\sigma$-algebra on $[0,1]$. More formally, $\mathcal{S}$ is the $\sigma$-algebra generated by cylinder sets of the form $A_{1} \times A_{2} \times \cdots \times A_{k} \times[0,1] \times[0,1] \times[0,1] \ldots$, where $A_{1}, A_{2}, \ldots, A_{k}$ are Borel subsets of $[0,1]$.

- The measure $\mu_{\mathbb{S}}$ on $(\mathbb{S}, \mathcal{S})$ is the extension of the Lebesgue measure to the infinite product space $(\mathbb{S}, \mathcal{S}$ ). Formally, it is the unique (by Kolmogorov's extension theorem) measure such that for all finite sequences of Borel subets $A_{1}, A_{2}, \ldots, A_{k}$ of $[0,1]$, we have $\mu_{\mathbb{S}}\left(A_{1} \times A_{2} \times \cdots \times A_{k} \times[0,1]^{\omega}\right)=\mu_{L}\left(A_{1}\right) \times$ $\mu_{L}\left(A_{2}\right) \times \ldots \times \mu_{L}\left(A_{k}\right) \times \mu_{L}([0,1]) \times \mu_{L}([0,1]) \cdots=\mu_{L}\left(A_{1}\right) \times \mu_{L}\left(A_{2}\right) \times \ldots \times$ $\mu_{L}\left(A_{k}\right)$

- The function $\pi_{U}$ returns the first element of the given sequence - that is, $\pi_{U}\left(\left(s_{1}, s_{2}, s_{3}, \ldots\right)\right)=s_{3}$.

- The functions $\pi_{L}$ and $\pi_{R}$ return the subsequences consisting of odd and even elements of the input sequence, respectively. Thus, $\pi_{L}\left(\left(s_{1}, s_{2}, s_{3}, s_{4}, \ldots\right)\right)=$ $\left(s_{1}, s_{3}, \ldots\right)$ and $\pi_{R}\left(\left(s_{1}, s_{2}, s_{3}, s_{4}, \ldots\right)\right)=\left(s_{2}, s_{4}, \ldots\right)$.

- The function :: interleaves the two input sequences, so that $\left(s_{1}, s_{2}, s_{3}, \ldots\right)::$ $\left(t_{1}, t_{2}, t_{3}, \ldots\right)=\left(s_{1}, t_{1}, s_{2}, t_{2}, s_{3}, t_{3}, \ldots\right)$.

Observe that the functions $\pi_{L}$ and $\pi_{R}$ return two disjoint infinite subsequences of the input sequence. This means that if we want to perform two random computations, but only have a single entropy $s=\left(s_{1}, s_{2}, s_{3}, s_{4} \ldots\right)$, we can perform the first computation with the sequence $\pi_{L}\left(\left(s_{1}, s_{2}, s_{3}, s_{4} \ldots\right)\right)=$ $\left(s_{1}, s_{3}, \ldots\right)$ and the second one with $\pi_{R}\left(\left(s_{1}, s_{2}, s_{3}, s_{4} \ldots\right)\right)=\left(s_{2}, s_{4}, \ldots\right)$ and no entropy component from the first computation will be reused in the second one. In other words, for each new random sample we will have a "fresh" value in the entropy.

Results presented in this paper will, however, only depend on the abstract definition of entropy space.

\subsection{Extended state space}

In order to define the operational semantics and the distributions induced by it, we need to extend the set of states $\Omega_{\sigma}$ with two exception states: $\downarrow$, denoting a failed hard constraint or an evaluation error, and $\uparrow$, denoting divergence. We 
denote this extended space by $\hat{\Omega}_{\sigma}$. A metric space on $\hat{\Omega}_{\sigma}$ is defined by extending the metric $d_{\sigma}$ on $\Omega_{\sigma}$ to $\hat{\Omega}_{\sigma}$ as follows:

$$
\hat{d}_{\sigma}\left(\sigma_{1}, \sigma_{2}\right)= \begin{cases}0 & \text { if } \sigma_{1}=\sigma_{2} \in\{\not, \uparrow\} \\ d_{\sigma}\left(\sigma_{1}, \sigma_{2}\right) & \text { if } \sigma_{1}, \sigma_{2} \in \Omega_{\sigma} \\ \infty & \text { otherwise. }\end{cases}
$$

It is easy to check that the extended metric space $\left(\hat{\Omega}_{\sigma}, \hat{d}_{\sigma}\right)$ is separable. The $\sigma$-algebra $\hat{\Sigma}_{\sigma}$ on $\hat{\Omega}_{\sigma}$ is then induced by the metric $\hat{d}_{\sigma}$ and $\left(\hat{\Omega}_{\sigma}, \hat{\Sigma}_{\sigma}\right)$ is the measurable space of all program states.

In the remainder of this section, we will use two operators to extend realvalued functions on $\Omega_{\sigma}$ to the state space $\hat{\Omega}_{\sigma}$ : for each function $f: \Omega_{\sigma} \rightarrow \overline{\mathbb{R}}_{+}$, the extended functions $\hat{f}, \check{f}: \hat{\Omega}_{\sigma} \rightarrow \overline{\mathbb{R}}_{+}$are defined as follows:

$$
\hat{f}(\tau)=\left\{\begin{array}{ll}
f(\tau) & \text { if } \tau \in \Omega_{\sigma} \\
0 & \text { otherwise }
\end{array} \text { and } \quad \check{f}(\tau)= \begin{cases}f(\tau) & \text { if } \tau \in \Omega_{\sigma} \\
1 & \text { if } \tau=\uparrow \\
0 & \text { otherwise. }\end{cases}\right.
$$

\subsection{Reduction relation}

To ensure that the entropy is split correctly between partial computations, we use continuations, similarly to [39]. A continuation is represented by a list of expressions that are to be evaluated after completing the current evaluation. We keep track of two distinct entropies: one for the current computation and one to be used when evaluating the continuation.

The reduction relation is defined as a binary relation on configurations, i.e., tuples of the form

$$
\left\langle\theta, C, K, \sigma, \theta_{K}, n, w\right\rangle
$$

where $C$ is the current program statement to be evaluated, $\sigma$ is the current program state, $K$ is the continuation, $\theta$ and $\theta_{K}$ are, respectively, the entropies to be used when evaluating $C$ and the continuation $K$; finally, $n \in \mathbb{N}$ is the number of reduction rules applied so far and $w \in \mathbb{R} \cap[0,1]$ is the weight of the current program run so far. In order to access elements of a configuration $\kappa$, we use functions, e.g., for $\kappa$ as given above weight $(\kappa)=w$ and state $(\kappa)=\sigma$. The reduction relation $\vdash$ is a binary relation on configurations where

$$
\underbrace{\left\langle\theta, C, K, \sigma, \theta_{K}, n, w\right\rangle}_{\text {configuration } \kappa} \vdash \underbrace{\left\langle\theta^{\prime}, C^{\prime}, K^{\prime}, \sigma^{\prime}, \theta_{K}^{\prime}, n^{\prime}, w^{\prime}\right\rangle}_{\text {configuration } \kappa^{\prime}}
$$

means that the configuration $\kappa$ reduces to configuration $\kappa^{\prime}$ in one step. Let $\vdash^{*}$ denote the reflexive and transitive closure of the reduction relation $\vdash$, i.e., $\kappa \vdash^{*} \kappa^{\prime}$ means that $\kappa$ reduces to $\kappa^{\prime}$ in zero or more reduction steps.

We present the reduction rules one-by-one for each syntactic construct of PL. The symbol $\downarrow$ means successful termination and $\downarrow$ is a special state reached after a failed observation or an execution error. 
Skip. As the skip statement cannot do anything, it has no reduction rule.

Divergence. The (diverge) rule states that the diverge statement reduces to itself indefinitely in any non-failure state $\sigma$ :

$$
\text { diverge } \frac{\sigma \neq \underline{z}}{\left\langle\theta \text {, diverge, } K, \sigma, \theta_{K}, n, w\right\rangle \vdash\left\langle\theta \text {, diverge, } K, \sigma, \theta_{K}, n+1, w\right\rangle}
$$

Assignment. The rule (assign) evaluates the expression $E$ in the current state $\sigma$ and sets the value of $x$ in the state to the outcome of this evaluation:

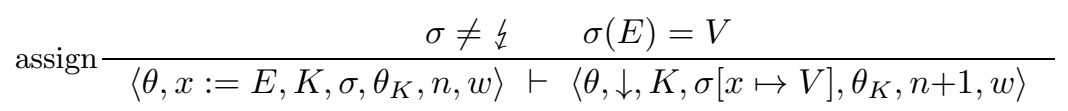

Random draw. The rule (draw) evaluates a random draw from a uniform distribution:

$$
\operatorname{draw} \frac{\sigma \neq \underline{z}}{\left\langle\theta, x: \approx U, K, \sigma, \theta_{K}, n, w\right\rangle \vdash\left\langle\pi_{R}(\theta), \downarrow, K, \sigma\left[x \mapsto \pi_{U}\left(\pi_{L}(\theta)\right)\right], \theta_{K}, n+1, w\right\rangle}
$$

The outcome of this random draw is determined by the entropy $\theta$ and is set to $\pi_{U}\left(\pi_{L}(\theta)\right)$, intuitively the first element of the "left" part of the entropy $\theta 6$. The value of the sampled variable is assigned to variable $x$. The weight $w$ is unchanged, as the density of the uniform distribution on $[0,1]$ is constant and equal to 1 for every point in the unit interval — as all outcomes are equally likely, there is no need to weigh the program runs.

Hard. The rules (condition-true) and (condition-false) evaluate a hard condition in an observe statement. If the condition is satisfied, (condition-true) returns the current state and weight unchanged, otherwise the state is set to the error state $\{$ by (condition-false):

$$
\begin{gathered}
\text { condition-true } \frac{\sigma \neq \downarrow}{\left\langle\theta, \text { observe }(\phi), K, \sigma, \theta_{K}, n, w\right\rangle \vdash\left\langle\theta, \downarrow, K, \sigma, \theta_{K}, n+1, w\right\rangle} \\
\text { condition-false } \frac{\sigma \neq \downarrow}{\left\langle\theta, \text { observe }(\phi), K, \sigma, \theta_{K}, n, w\right\rangle \vdash\left\langle\theta, \downarrow,[], \downarrow, \theta_{K}, n+1, w\right\rangle}
\end{gathered}
$$

Soft. The rule (score) evaluates its argument, a real number in the unit interval, and multiplies it by the weight of the current run so far:

$$
\operatorname{score} \frac{\sigma \neq \downarrow \quad v=\sigma(E) \in(0,1]}{\left\langle\theta, \operatorname{score}(E), K, \sigma, \theta_{K}, n, w\right\rangle \vdash\left\langle\theta, \downarrow, K, \sigma, \theta_{K}, n+1, w \cdot v\right\rangle}
$$

\footnotetext{
${ }^{6}$ If we set this value to just $\pi_{U}(\theta)$, we would lose the property that an already used "element" of the entropy cannot appear in the entropy in the subsequent configuration, because we do not know what parts of $\theta$ the value of $\pi_{U}(\theta)$ depends on. In the Hilbert cube implementation discussed before, $\pi_{U}(\theta)$ is equivalent to $\pi_{U}\left(\pi_{L}(\theta)\right)$ and "disjoint" from $\pi_{R}(\theta)$, but if we defined $\pi_{U}(\theta)$ to be, for instance, the second element of the sequence encoded by $\theta$, this would not be the case. Obviously, this does not matter in practice, as after the (draw) rule, the expression to be evaluated with entropy $\pi_{R}(\theta)$ is empty, but it is still elegant to keep this property.
} 
Sequencing. The rule (seq) is used to move statements from the current statement $C$ to the continuation $K$ :

$$
\operatorname{seq} \frac{\sigma \neq \downarrow}{\left\langle\theta, C_{1} ; C_{2}, K, \sigma, \theta_{K}, n, w\right\rangle \vdash\left\langle\pi_{L}(\theta), C_{1}, C_{2}:: K, \sigma, \pi_{R}(\theta):: \theta_{K}, n+1, w\right\rangle}
$$

If the current statement is a sequence of statements, (seq) splits it into the first statement $C_{1}$ and the sequence of remaining statements $C_{2}$ in such a way that $C_{1}$ itself is a single concrete statement and not a sequence of statements - in other words, $C_{1}$ is as small as possible. The expression $C_{1}$ is then retained as the current expression to be evaluated, while $C_{2}$ is pushed onto the top of the expression stack in the continuation $K$. The expression $C_{1}$ is evaluated with only the "left" part of the entropy $\theta$, and the right part is appended to the entropy of the continuation $K$; it is stored to be used later when $C_{2}$ is popped from the stack and evaluated. The reason that $C_{1}$ is required not to be a sequence is to ensure that there is a unique way to split the sequence of statements into $C_{1}$ and $C_{2}$. If the entropy could be split in different ways into sub-computations, this would make the semantics nondeterministic. Note that $C_{1}$ may be, e.g., an if-statement or a while loop which includes a sequence of statements as its sub-expression; we only require that it is not a sequence at the top level. The rule

$$
\operatorname{pop} \frac{\sigma \neq \downarrow}{\left\langle\theta, \downarrow, C:: K, \sigma, \theta_{K}, n, w\right\rangle \vdash\left\langle\pi_{L}\left(\theta_{K}\right), C, K, \sigma, \pi_{R}\left(\theta_{K}\right), n+1, w\right\rangle}
$$

is the dual of (seq). After the current statement has been completely evaluated, (pop) fetches the top statement $C$ from the continuation $K$ and sets it as the next statement to be evaluated. The unused part $\theta$ of the entropy used in evaluating the last expression is discarded and replaced by the left part of the continuation entropy $\theta_{K}$. If the evaluation started with an empty continuation, $\pi_{L}\left(\theta_{K}\right)$ will be the entropy "reserved" for evaluating $C$ as it was pushed on the continuation by (seq). Obviously, the entropy reserved for $C$ has to be removed from the entropy saved for evaluating the rest of the continuation, hence the latter is set to $\pi_{R}\left(\theta_{K}\right)$.

Conditional. The rules (if-true) and (if-false) are standard and self-explanatory.

$$
\begin{gathered}
\text { if-true } \frac{\sigma \neq \downarrow}{\left\langle\theta, \text { if }(\phi)\{C\}, K, \sigma, \theta_{K}, n, w\right\rangle \vdash\left\langle\theta, C, K, \sigma, \theta_{K}, n+1, w\right\rangle} \\
\text { if-false } \frac{\sigma \neq \downarrow}{\left\langle\theta, \text { if }(\phi)\{C\}, K, \sigma, \theta_{K}, n, w\right\rangle \vdash\left\langle\theta, \downarrow, K, \sigma, \theta_{K}, n+1, w\right\rangle}
\end{gathered}
$$

Loops. The (while-true) and (while-false) rules are standard too. If the loop-guard $\phi$ is true, the loop body is to be executed possibly followed by the loop itself. Otherwise the loop terminates. The (while-true) rule reads

$$
\frac{\sigma \neq \underline{z}}{\left\langle\theta, \text { while }(\phi)\{C\}, K, \sigma, \theta_{K}, n, w\right\rangle \vdash\left\langle\theta, C ; \text { while }(\phi)\{C\}, K, \sigma, \theta_{K}, n+1, w\right\rangle}
$$




$$
\text { while-false } \frac{\sigma \neq \underline{z} \quad \sigma(\phi)=\text { false }}{\left\langle\theta, \text { while }(\phi)\{C\}, K, \sigma, \theta_{K}, n, w\right\rangle \vdash\left\langle\theta, \downarrow, K, \sigma, \theta_{K}, n+1, w\right\rangle}
$$

The (final) rule is a dummy rule which applies to fully evaluated programs. It does nothing, except for increasing the step counter. Its purpose is to allow for reasoning about infinite evaluations, as explained later.

$$
\text { final } \frac{\sigma \neq \underline{\imath}}{\left\langle\theta, \downarrow,[], \sigma, \theta_{K}, n, w\right\rangle \vdash\left\langle\theta, \downarrow,[], \sigma, \theta_{K}, n+1, w\right\rangle}
$$

The initial configuration for program $C$ is of the form $\left\langle\theta, C\right.$, [], $\left.\sigma, \theta_{K}, 0,1\right\rangle$ with the initial statement $C$, the empty continuation, initial state $\sigma$, a zero step count and initial weight one. Note that if the initial continuation is [], the initial continuation entropy $\theta_{K}$ is irrelevant, as it can never be copied to the entropy of the current expression. A program is considered fully evaluated when the evaluation reaches a configuration where $C=\downarrow$ and $K=$ []. In this case, only the dummy (final) rule can be applied. Thus, if

$$
\left\langle\theta, C,[], \sigma, \theta_{K}, 0,1\right\rangle \vdash^{*}\left\langle\theta^{\prime}, \downarrow,[], \sigma^{\prime}, \theta_{K}^{\prime}, n, w\right\rangle
$$

we say that the program $C$ with initial state $\sigma$ under entropy $\theta$ terminates in $n$ steps in the state $\sigma^{\prime}$ with weight $w$.

Examples We demonstrate how the semantics works by revisiting two of the examples in Section 3 For clarity, we now add line numbers to programs and write $C_{i}$ for line $i$ of the given program $C$ and $C_{i, j}$ for the part of the program between lines $i$ and $j$. We also write $\pi_{d_{1}, \ldots, d_{n}}(\theta)$ (where $d_{1}, \ldots, d_{n} \in\{L, R\}$ ) for $\pi_{d_{1}}\left(\pi_{d_{2}} \ldots\left(\pi_{d_{n}}(\theta) \ldots\right)\right)$.

Example 5. We begin by revisiting the Bayesian linear regression example:

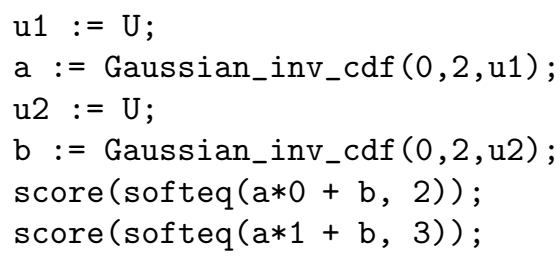

Let us suppose we want to evaluate this program with an empty initial state and with an entropy $\theta$ such that the two values sampled in the program (which are $\pi_{U}\left(\pi_{L, L}(\theta)\right)$ and $\pi_{U}\left(\pi_{L, L, R, R}(\theta)\right)$ ), are, respectively, 0.5 and $v$, where $v \in(0,1)$ is a value such that Gaussian_inv_cdf $(0,2, v)=2$. For the particular instantiation of the entropy space shown in Section 4.1, we have $\pi_{U}\left(\pi_{L, L}\left(\left(s_{1}, s_{2}, s_{3}, \ldots\right)\right)\right)=s_{1}$ and $\pi_{U}\left(\pi_{L, L, R, R}\left(\left(s_{1}, s_{2}, s_{3}, \ldots\right)\right)\right)=s_{13}$, so we can assume that $\theta$ is any infinite sequence $\left(s_{1}, s_{2}, s_{3}, \ldots\right)$ whose first element is 0.5 and thirteenth element is $v$. Note that Gaussian_inv_cdf $(0,2,0.5)=0$, because the Gaussian distribution is symmetric, so exactly half of the total probability mass is below the mean. 
The evaluation chain is shown below. We use colour to highlight states and scores which have changed from the previous configuration. Since the evaluation starts with a configuration with empty continuation, the initial continuation entropy $\theta_{K}$ is a "dummy" entropy whose values are irrelevant and do not affect the computation.

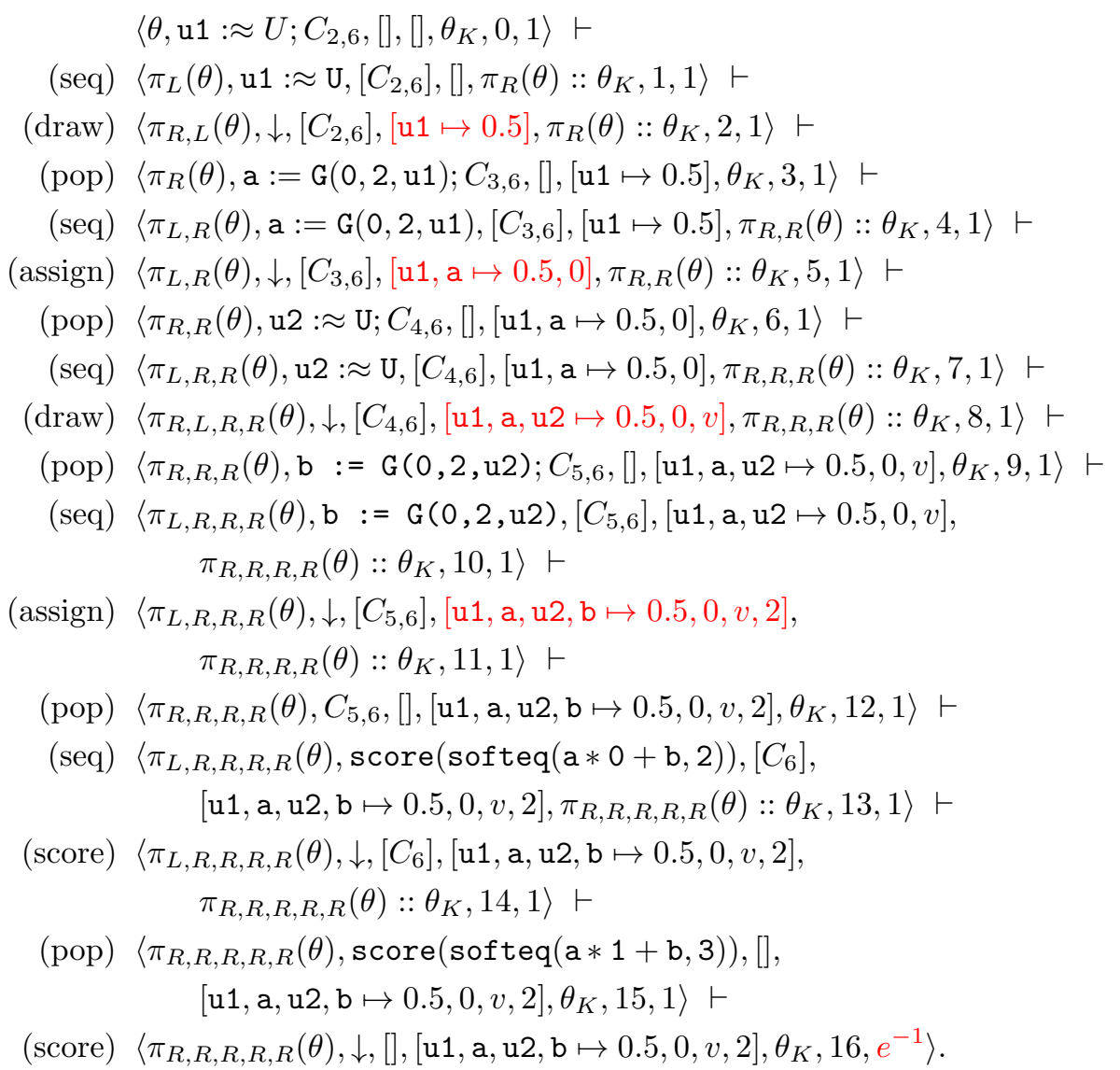

Hence, with the given entropy $\theta$, the program evaluates with score $e^{-1}$ to a state where $\mathrm{a}=0$ and $\mathrm{b}=0$.

Example 6. Let us now consider the program in Example 2:

$\mathrm{b}:=0$;

$\mathrm{k}:=0$;

while $(b=0)$

\{

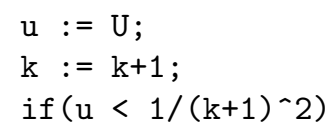




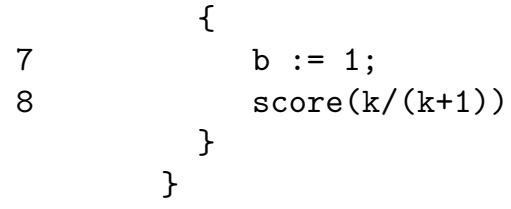

We want to compute the final state and weight for this program, assuming an empty initial state and an entropy $\theta$ such that the first value drawn (that is, $\left.\pi_{U}\left(\pi_{L, L, R, R}(\theta)\right)\right)$ is 0.1 , which also means the loop terminates after the first iteration. The evaluation proceeds as follows:

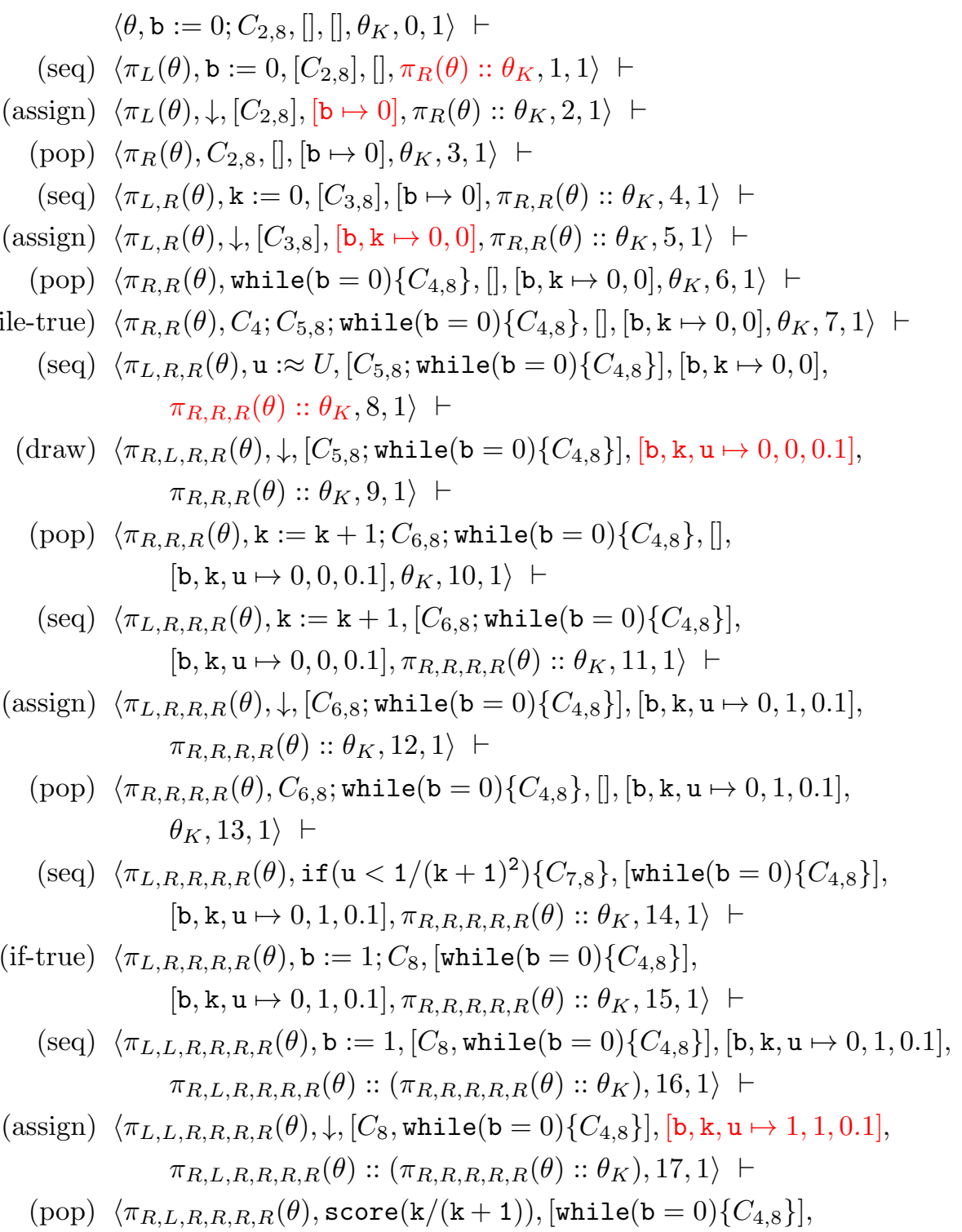




$$
\begin{gathered}
\left.[\mathrm{b}, \mathrm{k}, \mathrm{u} \mapsto 1,1,0.1], \pi_{R, R, R, R, R}(\theta):: \theta_{K}, 18,1\right\rangle \vdash \\
\text { (score) }\left\langle\pi_{R, L, R, R, R, R}(\theta), \downarrow,\left[\operatorname{while}(\mathrm{b}=0)\left\{C_{4,8}\right\}\right],[\mathrm{b}, \mathrm{k}, \mathrm{u} \mapsto 1,1,0.1],\right. \\
\left.\left(\pi_{R, R, R, R, R}(\theta):: \theta_{K}\right), 19,1 / 2\right\rangle \vdash \\
\text { (pop) }\left\langle\pi_{R, R, R, R, R}(\theta), \text { while }(\mathrm{b}=0)\left\{C_{4,8}\right\},[],[\mathrm{b}, \mathrm{k}, \mathrm{u} \mapsto 1,1,0.1], \theta_{K}, 20,1 / 2\right\rangle \vdash \\
\text { (while-false) }\left\langle\pi_{R, R, R, R, R}(\theta), \downarrow,[],[\mathrm{b}, \mathrm{k}, \mathrm{u} \mapsto 1,1,0.1], \theta_{K}, 21,1 / 2\right\rangle .
\end{gathered}
$$

Hence, the program evaluates to a state where $\mathrm{k}=1$ with score $1 / 2$.

\subsection{Measure on final program states}

As mentioned before, the operational semantics so far only defines the final state and weight for a single program execution, for a fixed entropy. We now explain how a probability distribution on final states can be obtained by integrating the semantics over the entropy space.

Two auxiliary functions. In order to define a probability distribution over the final program states, two auxiliary functions are technically convenient. The function $\mathbf{O}_{C}^{\sigma}: \mathbb{S} \rightarrow \hat{\Omega}_{\sigma}$ determines the final state of program $C$ with initial state $\sigma$ for entropy $\theta \in \mathbb{S}$. It is defined by:

$$
\begin{aligned}
& \mathbf{O}_{C}^{\uparrow}(\theta)=\uparrow \\
& \mathbf{O}_{C}^{\sigma}(\theta)= \begin{cases}\tau & \text { if }\left\langle\theta, C,[], \sigma, \theta_{K}, 0,1\right\rangle \vdash^{*}\left\langle\theta^{\prime}, \downarrow,[], \tau, \theta_{K}, n, w\right\rangle \text { and } \tau \neq \downarrow \\
\downarrow & \text { if }\left\langle\theta, C,[], \sigma, \theta_{K}, 0,1\right\rangle \vdash^{*}\left\langle\theta^{\prime}, C^{\prime}, K, \tau, \theta_{K}^{\prime}, n, w\right\rangle \nvdash \\
\uparrow & \text { otherwise. }\end{cases}
\end{aligned}
$$

The final state of program $C$ and proper initial state $\sigma$ (i.e., $\sigma \neq \uparrow$ and $\sigma \neq$ 々) with entropy $\theta$ equals state $\tau$ provided its execution ends in configuration $\langle\cdot,[], \tau, \cdots\rangle$. For instance, for the program $C$ and entropy $\theta$ from Example 6 , we have $\mathbf{O}_{C}^{[]}(\theta)=[\mathrm{b} \mapsto 1, \mathrm{k} \mapsto 1, \mathrm{u} \mapsto 0.1]$. If the evaluation reaches a configuration which cannot be reduced any further (e.g., due to a failed hard constraint), the final state equals the error state $\downarrow$. Note that this is also applicable to the initial state $\sigma=\xi$. Finally, if the evaluation can neither be completed nor reach an irreducible configuration, this means that the evaluation of program $C$ with state $\sigma$ diverges with entropy $\theta$. This results in the extended state $\uparrow$. For an example of a computation leading to such a state, consider Example 6 again. If we take an entropy $\theta^{\prime}$ such that the value sampled in line 4 in each iteration is 0.5 , the evaluation will never terminate and so we have $\mathbf{O}_{C}^{[]}\left(\theta^{\prime}\right)=\uparrow$. The final state of running $C$ from initial state $\downarrow$ is the initial state. This way of handling exceptions ensures compositionality. We omit $C$ and $\sigma$ as sub- and superscript if they are clear from the context.

Besides the final state obtained from a program's run, we need also the run's score. The function $\mathbf{S C}_{C}^{\sigma}: \mathbb{S} \rightarrow \mathbb{R}_{+}$yields the score of executing program $C$ from initial state $\sigma$ for a given entropy. This definition is a bit more complicated, due to the handling of diverging runs. A naive solution would be to define $\mathbf{S C}$ 
similarly to $\mathbf{O}$ and return 0 for diverging runs. This would, however, mean that the semantics would quietly ignore diverging runs, while a key motivation for this work is to handle divergence in the presence of soft conditioning in a meaningful way. Our proposal is to let the score for diverging runs be the limit of the weight $w$ as the number of steps $n$ goes to infinity. Formally, this is done as follows: Let us define an approximation function $\mathbf{S C}_{C}^{\sigma}: \mathbb{S} \times \mathbb{N} \rightarrow \mathbb{R}_{+}$, such that $\mathbf{S C}_{C}^{\sigma}(\theta, n)$ returns the score for program $C$ with entropy $\theta$ and initial state $\sigma$ after $n$ evaluation steps:

$$
\mathbf{S C}_{C}^{\sigma}(\theta, n)= \begin{cases}w & \text { if }\left\langle\theta, C,[], \sigma, \theta_{K}, 0,1\right\rangle \vdash^{*}\left\langle\theta^{\prime}, C^{\prime}, K, \tau, \theta_{K}^{\prime}, n, w\right\rangle \text { and } \tau \neq \downarrow \\ 0 & \text { otherwise. }\end{cases}
$$

The function $\mathbf{S C}_{C}^{\sigma}: \mathbb{S} \rightarrow \mathbb{R}_{+}$is now defined for proper state $\sigma$ as the limit, or equivalently infimum, of its $n$-the approximation:

$$
\mathbf{S C}_{C}^{\sigma}(\theta)=\lim _{n \rightarrow \infty} \mathbf{S C}_{C}^{\sigma}(\theta, n)=\inf _{n} \mathbf{S C}_{C}^{\sigma}(\theta, n)
$$

For the special cases $\sigma=\downarrow$ and $\sigma=\uparrow$ we define:

$$
\mathbf{S C}_{C}^{\uparrow}(\theta)=1 \text { and } \mathbf{S C}_{C}^{\xi}(\theta)=0
$$

Example \%. Let us revisit the program $C$ from Example 6 to show how the SC function works. The program terminates with score $\frac{1}{2}$ after 16 steps with the original entropy $\theta$ used in the example. Thus, $\mathbf{S C}_{C}^{[]}(\theta, 16)=\frac{1}{2}$. The final configuration reduces to itself by (diverge) infinitely many times, so we have $\mathbf{S C}_{C}^{[]}(\theta, n)=\frac{1}{2}$ for all $n \geq 16$. Thus, $\mathbf{S C}_{C}^{[]}(\theta)=\frac{1}{2}=\lim _{n \rightarrow \infty} \mathbf{S C}_{C}^{[]}(\theta, n)=\frac{1}{2}$. This is the same result which would be returned by a naive definition of $\mathbf{S C}$, similar to $\mathbf{O}$. However, if we use the entropy $\theta^{\prime}$ described above, where all values sampled are 0.5 , this is no longer the case. In this case, we have $\mathbf{S C}_{C}^{[]}(\theta, n)=1$ for all $n$, because the only score statement is never reached. Hence, $\mathbf{S C}_{C}^{[]}\left(\theta^{\prime}\right)=$ $\lim _{n \rightarrow \infty} \mathbf{S C}_{C}^{[]}\left(\theta^{\prime}, n\right)=1$, while the naive definition would return 0 .

Example 8. For a more illustrative example, we consider the trivial program $C^{\prime}$ :

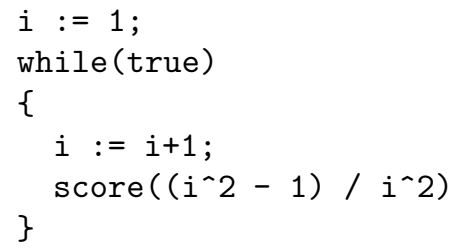

Now, for any $\theta$, we have $\mathbf{S C}_{C}^{[]}(\theta, n)=\frac{1}{2} \cdot \frac{i(n)+2}{i(n)+1}$, where $i(n)$ is the number of loop iterations completed after $n$ steps. As the second factor converges to 1 as $n$ (and so $i(n)$ ) goes to infinity, it follows that $\mathbf{S C}_{C}^{[]}(\theta)=\frac{1}{2}$, so the limit score is $\frac{1}{2}$ even though the program never terminates. 
Note that by the monotone convergence theorem, the limit of approximations always exists. Thus, SC is well-defined. This can be seen as follows. As scores are bounded by 1 , and no rule other than (score) affects the weight of a program run, a reduction step cannot increase the total score:

Lemma 7. $\kappa \vdash \kappa^{\prime}$ implies $\operatorname{weight}(\kappa) \geq \operatorname{weight}\left(\kappa^{\prime}\right)$.

Thus, scores are antitone: $k \geq n$ implies $\mathbf{S C}_{C}^{\sigma}(\theta, k) \leq \mathbf{S C}_{C}^{\sigma}(\theta, n)$. The monotone convergence theorem now yields:

Lemma 8. For each PL program $C$, state $\sigma$ and entropy $\theta, \lim _{n \rightarrow \infty} \mathbf{S C}_{C}^{\sigma}(\theta, n)$ exists and is finite.

To define the probability distribution of states as a Lebesgue integral involving functions $\mathbf{O}$ and $\mathbf{S C}$, these functions need to be shown to be measurable. Although this is a property satisfied by almost all functions used in practice and it is known to be hard to construct non-measurable functions - to construct nonLebesgue-measurable sets of reals, and hence non-measurable functions on reals, requires the Axiom of Choice [34] - measurability proofs tend to be lengthy and tedious. A detailed proof of measurability of functions similar to $\mathbf{O}_{C}$ and $\mathbf{S C}_{C}$, can be found in [5] when providing a semantics to a probabilistic functional programming language. More details are in Appendix E we summarise here the main things:

Lemma 9. For all $C$ and $\sigma \in \hat{\Omega}_{\sigma}$ :

(1) $\mathbf{O}_{C}^{\sigma}(\cdot)$ is $\mathcal{S} / \hat{\Omega}_{\sigma}$ measurable and $(2) \mathbf{S C}_{C}^{\sigma}(\cdot)$ is $\mathcal{S} / \mathcal{R}$ measurable.

Proof. (1) Analogous to the proof of Lemma 92 in [5], more details in Appendix $\mathbf{F}$ (2) Analogous to the proof of Lemma 93 in [5], it follows that $\mathbf{S C}_{C}^{\sigma}(\cdot, n)$ is $\mathcal{S} / \mathcal{R}$ measurable, for all $n$; more details in Appendix $\mathrm{F}$ The result now follows by the fact that point-wise limits of measurable real-valued functions are measurable.

Distribution over final program states. We are now in a position to define the distribution on final states in terms of the operational semantics. We first define the distribution on entropies $\langle C\rangle_{\sigma}: \mathcal{S} \rightarrow \mathbb{R}_{+}$, as an integral of score $\mathbf{S C}_{C}^{\sigma}$ with respect to the standard measure on entropy space:

$$
\langle C\rangle_{\sigma}(B)=\int_{B} \mathbf{S C}_{C}^{\sigma}(\theta) \mu_{\mathbb{S}}(d \theta) .
$$

For each measurable subset $B \in \mathcal{S}$ of the entropy space, $\langle C\rangle_{\sigma}(B)$ is the probability that if we run program $C$ with initial state $\sigma$, the random values sampled during execution will match some element of the set $B$ of entropies.

The probability distribution $\llbracket C \rrbracket_{\sigma}: \hat{\Sigma}_{\sigma} \rightarrow \mathbb{R}_{+}$on extended states can now be defined as the push-forward measure of $\langle C\rangle_{\sigma}$ with respect to $\mathbf{O}_{C}^{\sigma}(\theta)$ :

$$
\begin{array}{r}
\llbracket C \rrbracket_{\sigma}(A)=\langle C\rangle_{\sigma}\left(\left\{\theta \mid \mathbf{O}_{C}^{\sigma}(\theta) \in A\right\}\right)=\langle C\rangle_{\sigma}\left(\mathbf{O}_{C}^{\sigma-1}(A)\right) \\
=\int\left[\mathbf{O}_{C}^{\sigma}(\theta)(A)\right] \cdot \mathbf{S C}_{C}^{\sigma}(\theta) \mu_{\mathbb{S}}(d \theta) .
\end{array}
$$


For program $C$ with $\langle C\rangle_{\sigma}(\mathbb{S})>0$, this distribution can be normalised as follows:

$$
\llbracket \hat{C} \rrbracket_{\sigma}(A)=\frac{\llbracket C \rrbracket_{\sigma}(A)}{\langle C\rangle_{\sigma}(\mathbb{S})} .
$$

The measure $\llbracket C \rrbracket_{\sigma}(A)$ is a measure on $\left(\hat{\Omega}_{\sigma}, \hat{\Sigma}_{\sigma}\right)$. Let $\left.\llbracket C \rrbracket_{\sigma}\right|_{\Omega_{\sigma}}$ be this measure restricted to $\left(\Omega_{\sigma}, \Sigma_{\sigma}\right)$, i.e., the space of proper states without $\downarrow$ and $\uparrow$, such that $\left.\llbracket C \rrbracket_{\sigma}\right|_{\Omega_{\sigma}}(A)=\llbracket C \rrbracket_{\sigma}(A)$ for $A \subseteq \Sigma_{\sigma}$.

Example 9. We go back once again to the Bayesian linear regression program from Example 5. We will first compute the measure $\llbracket C \rrbracket_{\sigma}$ on program outcomes.

If the value sampled in line 1 is $v_{1}$ and the value sampled in line 3 is $v_{2}$, it follows from the operational semantics that the final state is $\left[\mathrm{u} 1 \mapsto v_{1}, \mathrm{a} \mapsto\right.$ $\left.\mathrm{G}\left(0,2, v_{1}\right), \mathrm{u} 2 \mapsto v_{2}, \mathrm{~b} \mapsto \mathrm{G}\left(0,2, v_{2}\right)\right]$ and the final score is

$$
e^{-\left(\mathrm{G}\left(0,2, v_{2}\right)-2\right)^{2}} e^{-\left(\mathrm{G}\left(0,2, v_{1}\right)+\mathrm{G}\left(0,2, v_{2}\right)-3\right)^{2}}=e^{-\left(\mathrm{G}\left(0,2, v_{2}\right)-2\right)^{2}-\left(\mathrm{G}\left(0,2, v_{1}\right)+\mathrm{G}\left(0,2, v_{2}\right)-3\right)^{2}} .
$$

Hence, for entropy $\theta$ with $\pi_{u}\left(\pi_{L, L}(\theta)\right)=v_{1}$ and $\pi_{u}\left(\pi_{L, L, R, R}(\theta)\right)=v_{2}$ :

$$
\begin{aligned}
\mathbf{O}_{C}^{[]}(\theta) & =\left[\mathrm{u} 1 \mapsto v_{1}, \mathrm{a} \mapsto \mathrm{G}\left(0,2, v_{1}\right), \mathrm{u} 2 \mapsto v_{2}, \mathrm{~b} \mapsto \mathrm{G}\left(0,2, v_{2}\right)\right] \\
\mathbf{S C}_{C}^{[]}(\theta) & =e^{-\left(\mathrm{G}\left(0,2, v_{2}\right)-2\right)^{2}-\left(\mathrm{G}\left(0,2, v_{1}\right)+\mathrm{G}\left(0,2, v_{2}\right)-3\right)^{2}} .
\end{aligned}
$$

This means that the integral $\int\left[\mathbf{O}_{C}^{[]}(\theta)(A)\right] \cdot \mathbf{S C}_{C}^{[]}(\theta) \mu_{\mathbb{S}}(d \theta)$ can be written as $\int f\left(\pi_{u}\left(\pi_{L, L}(\theta)\right), \pi_{u}\left(\pi_{L, L, R, R}(\theta)\right)\right) \mu_{\mathbb{S}}(d \theta)$, where

$$
\begin{gathered}
f\left(v_{1}, v_{2}\right)=\left[\left[\mathrm{u} 1, \mathrm{a}, \mathrm{u} 2, \mathrm{~b} \mapsto v_{1}, \mathrm{G}\left(0,2, v_{1}\right), v_{2}, \mathrm{G}\left(0,2, v_{2}\right)\right] \in A\right] \\
\cdot e^{-\left(\mathrm{G}\left(0,2, v_{2}\right)-2\right)^{2}-\left(\mathrm{G}\left(0,2, v_{1}\right)+\mathrm{G}\left(0,2, v_{2}\right)-3\right)^{2}} .
\end{gathered}
$$

By the definition of entropy, we have:

$$
\begin{aligned}
& \int f\left(\pi_{u}\left(\pi_{L, L}(\theta)\right), \pi_{u}\left(\pi_{L, L, R, R}(\theta)\right)\right) \mu_{\mathbb{S}}(d \theta) \\
= & \iint f\left(\pi_{u}\left(\pi_{L}\left(\theta_{L}\right)\right), \pi_{u}\left(\pi_{L, L, R}\left(\theta_{R}\right)\right)\right) \mu_{\mathbb{S}}\left(d \theta_{L}\right) \mu_{\mathbb{S}}\left(d \theta_{R}\right) \\
= & \left.\iiint \int f\left(\pi_{u}\left(\theta_{L, L}\right)\right), \pi_{u}\left(\pi_{L, L}\left(\theta_{R, R}\right)\right)\right) \mu_{\mathbb{S}}\left(d \theta_{L, L}\right) \mu_{\mathbb{S}}\left(d \theta_{R, L}\right) \mu_{\mathbb{S}}\left(d \theta_{L, R}\right) \mu_{\mathbb{S}}\left(d \theta_{R, R}\right) \\
= & \left.\iint f\left(\pi_{u}\left(\theta_{L, L}\right)\right), \pi_{u}\left(\pi_{L, L}\left(\theta_{R, R}\right)\right)\right) \mu_{\mathbb{S}}\left(d \theta_{L, L}\right) \mu_{\mathbb{S}}\left(d \theta_{R, R}\right) .
\end{aligned}
$$

By repeatedly applying the definition of entropy like above, we get:

$$
\begin{aligned}
& \left.\iint f\left(\pi_{u}\left(\theta_{L, L}\right)\right), \pi_{u}\left(\pi_{L, L}\left(\theta_{R, R}\right)\right)\right) \mu_{\mathbb{S}}\left(d \theta_{L, L}\right) \mu_{\mathbb{S}}\left(d \theta_{R, R}\right) \\
& \vdots \\
= & \left.\iint f\left(\pi_{u}\left(\theta_{L, L}\right)\right), \pi_{u}\left(\theta_{L, L, R, R}\right)\right) \mu_{\mathbb{S}}\left(d \theta_{L, L}\right) \mu_{\mathbb{S}}\left(d \theta_{L, L, R, R}\right) \\
= & \int_{[0,1]} \int_{[0,1]} f\left(v_{1}, v_{2}\right) \mu_{L}\left(d v_{1}\right) \mu_{L}\left(d v_{2}\right) .
\end{aligned}
$$


Thus,

$$
\begin{array}{r}
\llbracket C \rrbracket_{\sigma}(A)=\int_{(0,1)} \int_{(0,1)}\left[\left[\mathrm{u} 1 \mapsto v_{1}, \mathrm{a} \mapsto \mathrm{G}\left(0,2, v_{1}\right), \mathrm{u} 2 \mapsto v_{2}, \mathrm{~b} \mapsto \mathrm{G}\left(0,2, v_{2}\right)\right] \in A\right] \\
\cdot e^{-\left(\mathrm{G}\left(0,2, v_{2}\right)-2\right)^{2}-\left(\mathrm{G}\left(0,2, v_{1}\right)+\mathrm{G}\left(0,2, v_{2}\right)-3\right)^{2}} \mu_{L}\left(d v_{1}\right) \mu_{L}\left(d v_{2}\right) .
\end{array}
$$

Now, suppose that $A$ is a set of states such that $\mathrm{a}<0$ and $\mathrm{b}<0$. Then:

$$
\begin{aligned}
& \left.\llbracket C \rrbracket_{\sigma}(A)=\int_{(0,1)} \int_{(0,1)}\left[\mathrm{G}\left(0,2, v_{1}\right)<0\right]\left[\mathrm{G}\left(0,2, v_{2}\right)\right]<0\right] \\
& \cdot e^{-\left(\mathrm{G}\left(0,2, v_{2}\right)-2\right)^{2}-\left(\mathrm{G}\left(0,2, v_{1}\right)+\mathrm{G}\left(0,2, v_{2}\right)-3\right)^{2}} \mu_{L}\left(d v_{1}\right) \mu_{L}\left(d v_{2}\right) .
\end{aligned}
$$

Like in Example 1, this expression can be rewritten as a double integral of Gaussian densities over the real line:

$$
\begin{aligned}
\llbracket C \rrbracket_{\sigma}(A) & =\iint\left[x_{1}<0\right]\left[x_{2}<0\right] \cdot e^{-p(\boldsymbol{x})} \cdot \mathbf{G}_{p d f}\left(0,2, x_{1}\right) \cdot \mathbf{G}_{p d f}\left(0,2, x_{2}\right) \mu_{L}\left(d x_{1}\right) \mu_{L}\left(d x_{2}\right) \\
& =\int_{(-\infty, 0)} \int_{(-\infty, 0)} \cdot e^{-p(\boldsymbol{x})} \cdot \mathbf{G}_{p d f}\left(0,2, x_{1}\right) \cdot \mathbf{G}_{p d f}\left(0,2, x_{2}\right) \mu_{L}\left(d x_{1}\right) \mu_{L}\left(d x_{2}\right)
\end{aligned}
$$

where $e^{-p(\boldsymbol{x})}=e^{-\left(x_{2}-2\right)^{2}-\left(x_{1}+x_{2}-3\right)^{2}}$. Let us now compute the normalising constant $\langle C\rangle_{\sigma}(\mathbb{S})$. By a similar reasoning as above, we get:

$$
\begin{aligned}
\langle C\rangle_{\sigma}(\mathbb{S}) & =\int \mathbf{S C}_{C}^{\sigma}(\theta) \mu_{\mathbb{S}}(d \theta) \\
& =\int_{(0,1)} \int_{(0,1)} e^{-\left(\mathrm{G}\left(0,2, v_{2}\right)-2\right)^{2}-\left(\mathrm{G}\left(0,2, v_{1}\right)+\mathrm{G}\left(0,2, v_{2}\right)-3\right)^{2}} \mu_{L}\left(d v_{1}\right) \mu_{L}\left(d v_{2}\right) \\
& =\iint e^{-p(\boldsymbol{x})} \cdot \mathrm{G}_{p d f}\left(0,2, x_{1}\right) \cdot \mathrm{G}_{p d f}\left(0,2, x_{2}\right) \mu_{L}\left(d x_{1}\right) \mu_{L}\left(d x_{2}\right)
\end{aligned}
$$

Hence, the normalised semantics $\llbracket \hat{C} \rrbracket_{\sigma}(A)$ applied to the above set $A$ is:

$$
\llbracket \hat{C} \rrbracket_{\sigma}(A)=\frac{\int_{(-\infty, 0)} \int_{(-\infty, 0)} e^{-p(\boldsymbol{x})} \cdot \mathbf{G}_{p d f}\left(0,2, x_{1}\right) \cdot \mathbf{G}_{p d f}\left(0,2, x_{2}\right) \mu_{L}\left(d x_{1}\right) \mu_{L}\left(d x_{2}\right)}{\iint e^{-p(\boldsymbol{x})} \cdot \mathbf{G}_{p d f}\left(0,2, x_{1}\right) \cdot \mathbf{G}_{p d f}\left(0,2, x_{2}\right) \mu_{L}\left(d x_{1}\right) \mu_{L}\left(d x_{2}\right)} .
$$

\subsection{Expectations}

The weakest preexpectation semantics determines the expected value of an arbitrary measurable function $f$ on states with respect to a program. We can also obtain such expected value by integrating $f$ with respect to the measure $\llbracket C \rrbracket_{\sigma}(A)$ defined just above. By change of variable, this integral can be easily transformed into an integral with respect to the default measure on entropies.

Lemma 10. For all measurable $f$,

$$
\left.\int f(\tau) \llbracket C \rrbracket_{\sigma}\right|_{\Omega_{\sigma}}(d \tau)=\int \hat{f}\left(\mathbf{O}_{C}^{\sigma}(\theta)\right) \cdot \mathbf{S C}_{C}^{\sigma}(\theta) \mu_{\mathbb{S}}(d \theta) .
$$


Proof.

$$
\begin{aligned}
\left.\int f(\tau) \llbracket C \rrbracket_{\sigma}\right|_{\Omega_{\sigma}}(d \tau) & =\int \hat{f}(\tau) \llbracket C \rrbracket_{\sigma}(d \tau) \\
\text { (by property of the pushforward) } & =\int \hat{f}\left(\mathbf{O}_{C}^{\sigma}(\theta)\right)\langle C\rangle_{\sigma}(d \theta) \\
\text { (by Radon-Nikodým theorem) } & =\int \hat{f}\left(\mathbf{O}_{C}^{\sigma}(\theta)\right) \mathbf{S C}_{C}^{\sigma}(\theta) \mu_{\mathbb{S}}(d \theta) .
\end{aligned}
$$

Example 10. Let us compute the expected value of the variable a in the Bayesian linear regression example. To this end, we take a function $f$ such that $f(\sigma)=$ $\sigma(\mathrm{a})$ if $\mathrm{a} \in \operatorname{dom}(\sigma)$ and $f(\sigma)=0$ otherwise. By a similar reasoning as in Example 9, we get:

$$
\begin{aligned}
& \int f(\tau) \llbracket C \rrbracket_{[]} \mid \Omega_{\sigma}(d \tau) \\
= & \int \hat{f}\left(\mathbf{O}_{C}^{[]}(\theta)\right) \cdot \mathbf{S C}_{C}^{[]]}(\theta) \mu_{\mathbb{S}}(d \theta) \\
= & \int_{(0,1)} \int_{(0,1)} \hat{f}\left(\left[\mathrm{u} 1 \mapsto v_{1}, \mathrm{a} \mapsto \mathrm{G}\left(0,2, v_{1}\right), \mathrm{u} 2 \mapsto v_{2}, \mathrm{~b} \mapsto \mathrm{G}\left(0,2, v_{2}\right)\right]\right) \\
= & \int_{(0,1)} \int_{(0,1)} \mathrm{G}\left(0,2, v_{1}\right) \cdot e^{-\left(\mathrm{G}\left(0,2, v_{2}\right)-2\right)^{2}-\left(\mathrm{G}\left(0,2, v_{1}\right)+\mathrm{G}\left(0,2, v_{2}\right)-3\right)^{2}-\left(\mathrm{G}\left(0,2, v_{1}\right)+\mathrm{G}\left(0,2, v_{2}\right)-3\right)^{2}} \mu_{L}\left(d v_{1}\right) \mu_{L}\left(d v_{2}\right)
\end{aligned}
$$

This is the same result as the one we obtain using wp in Example 1 .

Example 11. Let us now revisit the program from Example 2 and calculate the expected value of the constant function $f(\sigma)=1$ with respect to the program using the operational semantics. To this end, we need to calculate $\int \hat{f}\left(\mathbf{O}_{C}^{[]}(\theta)\right)$. $\mathbf{S C}_{C}^{[]}(\theta) \mu_{\mathbb{S}}(d \theta)$ for the given program $C$. By evaluating the first two statements in the program, like in Example [5, we can check that $\mathbf{O}_{C}^{[]}(\theta)=\mathbf{O}_{C^{\prime}}^{\sigma}\left(\pi_{R, R}(\theta)\right)$ and $\mathbf{S C}_{C}^{[]}(\theta)=\mathbf{S C}_{C^{\prime}}^{\sigma}\left(\pi_{R, R}(\theta)\right)$, where $C^{\prime}=$ while $(\mathrm{b}=0)\left\{C^{\prime \prime}\right\}\left(C^{\prime \prime}\right.$ being the loop body) and $\sigma=[\mathrm{b} \mapsto 0, \mathrm{k} \mapsto 0]$. It follows from the properties of entropy that $\int \hat{f}\left(\mathbf{O}_{C^{\prime}}^{\sigma}\left(\pi_{R, R}(\theta)\right)\right) \cdot \mathbf{S C}_{C^{\prime}}^{\sigma}\left(\pi_{R, R}(\theta)\right) \mu_{\mathbb{S}}(d \theta)=\int \hat{f}\left(\mathbf{O}_{C^{\prime}}^{\sigma}(\theta)\right) \cdot \mathbf{S C}_{C^{\prime}}^{\sigma}(\theta) \mu_{\mathbb{S}}(d \theta)$.

Now, let $C_{n}^{\prime}=w_{\text {wile }}^{n}(\mathrm{~b}=0)\left\{C^{\prime \prime}\right\}$. We can show (using Proposition 3 from Appendix D and the Beppo Levi's theorem) that

$$
\int \hat{f}\left(\mathbf{O}_{C^{\prime}}^{\sigma}(\theta)\right) \cdot \mathbf{S C}_{C^{\prime}}^{\sigma}(\theta) \mu_{\mathbb{S}}(d \theta)=\sup _{n} \int \hat{f}\left(\mathbf{O}_{C_{n}^{\prime}}^{\sigma}(\theta)\right) \cdot \mathbf{S C}_{C_{n}^{\prime}}^{\sigma}(\theta) \mu_{\mathbb{S}}(d \theta) .
$$

Since $\hat{f}$ has value 1 on all proper states and is 0 on state $\uparrow, \hat{f}\left(\mathbf{O}_{C_{n}^{\prime}}^{\sigma}(\theta)\right)=1$ if $\mathbf{O}_{C^{\prime}}^{\sigma}(\theta)$ is a proper state (that is, if $C^{\prime}$ terminates with initial state $\sigma$ and entropy $\theta$ ) and $\hat{f}\left(\mathbf{O}_{C_{n}^{\prime}}^{\sigma}(\theta)\right)=0$ if $C^{\prime}$ does not terminate with $\theta$. Thus, $\hat{f}\left(\mathbf{O}_{C_{n}^{\prime}}^{\sigma}(\theta)\right)=[\theta \in$ 
$\left.S_{1}\right]+\left[\theta \in S_{2}\right]+\ldots+\left[\theta \in S_{n-1}\right]$, where $S_{i}$ is the set of entropies resulting in termination after exactly $i$ iteration 7 .

The score is only multiplied by $\frac{\mathrm{k}}{\mathrm{k}+1}$ in the last iteration, after which the guard is satisfied. As long as the guard of the while-loop is false, the score stays at 1 . Thus, we have $\mathbf{S C}_{C}^{[]}(\theta)=\left[\theta \in S_{1}\right] \cdot \frac{1}{2}+\left[\theta \in S_{2}\right] \cdot \frac{2}{3}+\ldots+\left[\theta \in S_{n}\right] \cdot \frac{n}{n+1}+[\theta \notin$ $S_{1} \cup \ldots \cup S_{n} 8$.

Therefore, for each $n$,

$$
\begin{aligned}
\int \hat{f}\left(\mathbf{O}_{C_{n}^{\prime}}^{\sigma}(\theta)\right) \cdot \mathbf{S C}_{C_{n}^{\prime}}^{\sigma}(\theta) \mu_{\mathbb{S}}(d \theta) & =\int \sum_{k=1}^{n-1} \frac{k}{k+1}\left[\theta \in S_{k}\right] \mu_{\mathbb{S}}(d \theta) \\
& =\sum_{k=1}^{n-1} \frac{k}{k+1} \int\left[\theta \in S_{k}\right] \mu_{\mathbb{S}}(d \theta)
\end{aligned}
$$

Now we need to calculate $\int\left[\theta \in S_{k}\right] \mu_{\mathbb{S}}(d \theta)$ for each $k$. Observe that whether $\theta \in S_{k}$, depends only on parts of $\theta$ which are sampled from (that is, on subentropies to which $\pi_{U}$ is applied). The value of $\left[\theta \in S_{k}\right]$ depends only on the sub-entropies $\pi_{p_{1}}(\theta), \ldots, \pi_{p_{k}}(\theta)$, where $p_{1}, \ldots, p_{k}$ are the paths leading to values sampled in subsequent iterations. An entropy $\theta$ leads to termination in the $k$-th step if $\pi_{U}\left(\pi_{p_{1}}(\theta)\right) \geq \frac{1}{4}, \ldots, \pi_{U}\left(\pi_{p_{k-1}}(\theta)\right) \geq \frac{1}{k^{2}}$ and $\pi_{U}\left(\pi_{p_{k}}(\theta)\right) \leq \frac{1}{(k+1)^{2}}$. Thus, by the definition of entropy, we have

$$
\begin{aligned}
\int\left[\theta \in S_{k}\right] \mu_{\mathbb{S}}(d \theta)=\int\left[\pi_{U}\left(\pi_{p_{1}}(\theta)\right) \geq \frac{1}{4}\right] \ldots \cdot\left[\pi_{U}\left(\pi_{p_{k-1}}(\theta)\right) \geq \frac{1}{k^{2}}\right] \\
\cdot\left[\pi_{U}\left(\pi_{p_{k}}(\theta)\right) \leq \frac{1}{(k+1)^{2}}\right] \mu_{\mathbb{S}}(\theta) \\
=\int \cdots \int\left[\pi_{U}\left(\pi_{p_{1}}(\theta)\right) \geq \frac{1}{4}\right] \ldots \cdot\left[\pi_{U}\left(\pi_{p_{k-1}}(\theta)\right) \geq \frac{1}{k^{2}}\right] \\
\cdot\left[\pi_{U}\left(\pi_{p_{k}}(\theta)\right) \leq \frac{1}{(k+1)^{2}}\right] \mu_{\mathbb{S}}\left(\theta_{p_{1}}\right) \ldots \mu_{\mathbb{S}}\left(\theta_{p_{k}}\right) \\
=\int \ldots \int\left[v_{1} \geq \frac{1}{4}\right] \ldots \cdot\left[v_{k-1} \geq \frac{1}{k^{2}}\right] \\
\quad \cdot\left[v_{k} \leq \frac{1}{(k+1)^{2}}\right] \mu_{L}\left(d v_{1}\right) \ldots \mu_{L}\left(d v_{k}\right) \\
=\left(\prod_{i=1}^{k-1} \frac{(i+1)^{2}-1}{(i+1)^{2}}\right) \cdot \frac{1}{(k+1)^{2}} \\
=\frac{1}{2} \cdot \frac{1}{k \cdot(k+1)} .
\end{aligned}
$$

$\overline{7}$ The reason the last set is $S_{n-1}$ and not $S_{n}$ is that while ${ }^{1}(\phi)\left\{C^{\prime \prime}\right\}=C^{\prime \prime}$; diverge if $\phi$ is true, so while $e^{n}(\theta)\left\{C^{\prime \prime}\right\}$ only terminates if the loop body is executed at most $n-1$ times.

8 This time, the last set is $S_{n}$, because the score statement will be executed even if the loop body is followed by diverge. 
Hence, $\sum_{k=1}^{n-1} \frac{k}{k+1} \int\left[\theta \in S_{k}\right] \mu_{\mathbb{S}}(d \theta)=\frac{1}{2} \cdot \sum_{k=1}^{n-1} \frac{1}{(k+1)^{2}}$, so we have

$$
\begin{aligned}
\int \hat{f}\left(\mathbf{O}_{C}^{\llbracket]}(\theta)\right) \cdot \mathbf{S C}_{C}^{\llbracket]}(\theta) \mu_{\mathbb{S}}(d \theta) & =\int \hat{f}\left(\mathbf{O}_{C^{\prime}}^{\sigma}(\theta)\right) \cdot \mathbf{S C}_{C^{\prime}}^{\sigma}(\theta) \mu_{\mathbb{S}}(d \theta) \\
& =\sup _{n} \int \hat{f}\left(\mathbf{O}_{C_{n}^{\prime}}^{\sigma}(\theta)\right) \cdot \mathbf{S C}_{C_{n}^{\prime}}^{\sigma}(\theta) \mu_{\mathbb{S}}(d \theta) \\
& =\frac{1}{2} \sum_{k=1}^{\infty} \frac{1}{k \cdot(k+1)} \\
& =\frac{\pi^{2}}{12}-\frac{1}{2}
\end{aligned}
$$

This is exactly the result we obtained with the weakest preexpectation semantics in Example 2. The correspondence between the weakest preexpectation and operational semantics is the topic of the next section.

\section{Equivalence of wp and operational semantics}

The aim of this section is to show that the weakest preexpectation semantics of PL is equivalent to its operational semantics. This property is formalised by two theorems which relate the wp and wlp semantics to the operational semantics. The first result asserts that the expected value of an arbitrary function $f$ defined by the weakest preexpectation operator equals the expected value of $f$ computed as an integral of $f$ with respect to the distribution induced by the operational semantics.

Theorem 1. For all measurable functions $f: \Omega_{\sigma} \rightarrow \overline{\mathbb{R}}_{+}$, PL programs $C$ and initial states $\sigma \in \Omega_{\sigma}$ :

$$
\mathrm{wp} \llbracket C \rrbracket(f)(\sigma)=\int f(\tau) \llbracket C \rrbracket_{\sigma}(d \tau) .
$$

Proof. By Lemma 10, it suffices to prove that for all $f$ :

$$
\int \hat{f}\left(\mathbf{O}_{C}^{\sigma}(\theta)\right) \cdot \mathbf{S C}_{C}^{\sigma}(\theta) \mu_{\mathbb{S}}(d \theta)=\operatorname{wp} \llbracket C \rrbracket(f)(\sigma) .
$$

This can be proven by induction on the structure of $C$. The detailed proof can be found in Appendix $E$. The proof makes use of several compositionality properties of the operational semantics and properties of finite approximations of whileloops, which are also proven in the appendix. A key insight used in the proof is that Beppo Levi's theorem can be used to express the expectation of $f$ with respect to a while-loop as the limit of expectations of $f$ with respect to finite approximations of the loop.

The second main theorem of this paper states that the weakest liberal preexpectation of a non-negative function $f$ bounded by 1 is equivalent to the expected value of $f$ with respect to the distribution defined by the operational semantics plus the probability of divergence weighted by scores. 
Theorem 2. For every measurable non-negative function $f: \Omega_{\sigma} \rightarrow \overline{\mathbb{R}}_{+}$with $f(\sigma) \leq 1$ for all states $\sigma, P L$ program $C$ and initial state $\sigma \in \Omega_{\sigma}$ :

$$
\mathrm{wl} \llbracket \llbracket \rrbracket(f)(\sigma)=\left.\int f(\tau) \cdot \llbracket C \rrbracket_{\sigma}\right|_{\Omega_{\sigma}}(d \tau)+\underbrace{\int\left[\mathbf{O}_{C}^{\sigma}(\theta)=\uparrow\right] \cdot \mathbf{S C}_{C}^{\sigma}(\theta) \mu_{\mathbb{S}}(d \theta)}_{\begin{array}{c}
\text { probability of divergence } \\
\text { multiplied by the score }
\end{array}} .
$$

Proof. By induction on the structure of $C$. Details in Appendix E

Corollary 1. For every PL program $C$ and state $\sigma$ :

$$
\mathrm{wlp} \llbracket C \rrbracket(1)(\sigma)=\int \mathbf{S C}_{C}^{\sigma}(\theta) \mu_{\mathbb{S}}(d \theta) .
$$

\section{Related work}

Semantics of languages for Bayesian inference. Research on the semantics of probabilistic programs dates back to the pioneering work by Saheb-Djahromi 32] and Kozen [24], among others. However, this early work is mostly motivated by applications such as the analysis of randomised algorithms, so the languages involved mostly only supported discrete distributions and did not allow conditioning.

A recent explosion of popularity of machine learning, and the rise of probabilistic programming as a tool for Bayesian inference, have sparked a new line of work on semantics of languages with continuous random draws and conditioning. An early example of such work is the paper by Park et al. [31], who present an operational semantics for a higher-order language with conditioning, parametrised by an infinite trace of random values. Borgström et al. [6] define a denotational semantics of a first-order language with both discrete and continuous distributions, which also supports conditioning, including zero-probability observations. Nori et al. [28] define a denotational semantics of an imperative language with (hard) conditioning, similar to the weakest preexpectation semantics; they however do not consider possible program divergence. Toronto et al. 37] present a denotational semantics for a first-order functional language which interprets programs as deterministic functions on the source of randomness. Huang and Morrisett [16] define a semantics for a first-order language, restricted to computable operations. Heunen et at. 15] present a denotational semantics of a higher-order functional language with continuous random draws and conditioning. They manage to overcome the well-known problem with measurability of higher-order function application [2] by replacing standard Borel spaces with so-called quasi-Borel spaces. This idea, simplifying the authors' previous work [35], has since gained a lot of attraction in the community: Ścibior et al. 33] use quasi-Borel spaces to prove correctness of sampling-based inference algorithms, while Vákár et al. 38] define a domain theory for higher-order functional probabilistic programs, which extends the quasi-Borel space approach to 
programs with higher-order recursion and recursive types. A different approach is followed in a recent paper by Dahlqvist and Kozen [9], who define a semantics of a probabilistic language with conditioning in terms of Banach spaces.

The operational semantics presented in this chapter is strongly inspired by the semantics of Borgström et al. [5] and Wand et al. [39], both defined for functional programs. The former define a measure on program outcomes by integrating functions similar to our $\mathbf{O}$ and $\mathbf{S C}$, defined in terms of an operational semantics, with respect to a stock measure on traces of random values. The latter use a similar approach, but define their operational semantics in terms of infinite entropies instead of finite traces, and use continuations to fix evaluation order and split entropies between continuations consistently.

Program divergence. Another line of research on probabilistic programs, coming mostly from the algorithms and program verification community and inspired by earlier papers by Kozen 24] and McIver et al. [27], has focused on extending Dijkstra's weakest precondition calculus to probabilistic programs. In this line of work, correct handling of diverging programs has been a key issue from the start. Recent developments [13, 22, 7] focus on problems such as analysing runtimes, almost-sure termination (and variants thereof) and outcomes of algorithms. A weakest preexpectation semantics for recursive imperative probabilistic programs is given by Olmedo et al. 30]. Olmedo et al. 29] also extend the weakest preexpectation calculus to programs with hard conditioning and possible divergence, but their semantics only supports discrete distributions.

Combining continuous distributions, conditioning and divergence. The issue of program divergence has so far mostly been disregarded when defining semantics of Bayesian probabilistic programs, with most authors assuming that their semantics is only applicable to almost-surely terminating programs. Conversely, semantics designed to handle diverging programs usually did not support conditioning, and when they did, they were not applicable to programs with continuous distributions.

To our best knowledge, the only existing semantics supporting the combination of divergence, continuous random draws, and conditioning is the recent work by Bichsel et al. [3] . The authors define a semantics of an imperative probabilistic language with continuous and discrete distributions and hard conditioning, in which the probability of failing a hard constraint, the probability of an execution error and the probability of divergence are defined explicitly. The semantics calculates probability measures on final program states and the above exceptions are treated as special states, like $\underline{y}$ and $\uparrow$ in our semantics.

Technically, the semantics in 3] is a superset of our semantics. A normalised expectation of the form $\frac{\mathrm{wp} \llbracket C \rrbracket(f)\left(\sigma_{0}\right)}{\mathrm{w} 1 \mathrm{p} \llbracket C \rrbracket(1)\left(\sigma_{0}\right)}$ can be defined in their semantics as

$$
\frac{\int_{\Omega_{\sigma}} f(\tau) \llbracket C \rrbracket\left(\sigma_{0}\right)(d \tau)}{\llbracket C \rrbracket\left(\sigma_{0}\right)\left(\Omega_{\sigma}\right)+\llbracket C \rrbracket\left(\sigma_{0}\right)(\uparrow)}
$$

where $\llbracket C \rrbracket\left(\sigma_{0}\right)$ is the measure on final states of program $C$ with initial state $\sigma_{0}$, as defined by the semantics, and $\Omega_{\sigma}$ is the set of proper states (excluding errors and 
divergence). However, we believe that extending the well-studied framework of weakest preexpectations to the continuous case is still a significant contribution, as it allows using established techniques, not applicable to the semantics in [3], to analyse programs with continuous distributions, conditioning, in the presence of possible program divergence.

\section{$7 \quad$ Epilogue}

In this paper, we have considered a probabilistic while-language that contains three important ingredients: (a) sampling from continuous probability distributions, (b) soft and hard conditioning, and (c) program divergence. We have provided a weakest (liberal) preexpectation semantics for our language and showed that soft conditioning can be encoded by hard conditioning. The wp-semantics is complemented by an operational semantics using the concept of entropies. The main results of this paper are the correspondence theorem between the wpsemantics (and wlp-semantics) and the operational semantics. The paper has been written in a tutorial-like manner with various illustrative examples.

Let us conclude with a short discussion. The interplay between divergence and conditioning is intricate. For the discrete probabilistic setting, this has been extensively treated in [29]. Intuitively speaking, the problem is how conditioning is taken into account by program runs that diverge and never reach the score statement. Consider the program:

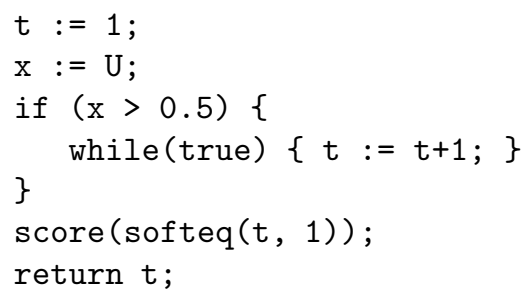

This program terminates with probability $1 / 2$ with $t=1$, and with the same probability diverges increasing $t$ ad infinitum. One would perhaps expect the expected value of $t$ to be 1 , as the possibility of $t$ going to infinity should be discarded by the score statement. However, for any function $f$, we have $\operatorname{wp}($ while $($ true $)\{t=t+1\})(f)=0$ and wlp(while(true) $\{t=t+1\})(f)=1$. Hence, the expected value of $t$ (for the empty initial state) will be:

$$
\frac{\mathrm{wp} \llbracket C \rrbracket(\lambda \sigma \cdot t)([])}{\mathrm{wlp} \llbracket C \rrbracket(\lambda \sigma . t)([])}=\frac{1 / 2}{1}=\frac{1}{2} .
$$

\section{A Basics of measure theory}

This section presents the basic definitions of measure theory used throughout this of the paper. For a more thorough introduction to measure theory, please consult one of the standard textbooks such as [4]. 


\section{Measurable spaces}

Definition 2. A $\sigma$-algebra $\Sigma$ on a set $\Omega$ is a set consisting of subsets of $\Omega$ which satisfies the following properties:

$-\emptyset \in \Sigma$

- If $A \in \Sigma$, then $\Omega \backslash A \in \Sigma$ (closure under complements)

- If $A_{i} \in \Sigma$ for all $i \in \mathbb{N}$, then $\bigcup_{i \in \mathbb{N}} A_{i} \in \Sigma$ (closure under countable unions)

The tuple $(\Omega, \Sigma)$ of a set $\Omega$ and its $\sigma$-algebra $\Sigma$ is called a measurable space. $A$ set $A \in \Sigma$ is called a measurable set.

Definition 3. A $\sigma$-algebra on a set $\Omega$ generated by a set $S$ of subsets of $\Omega$ is the smallest $\sigma$-algebra containing $S$.

Definition 4. A countably generated $\sigma$-algebra on $\Omega$ is a $\sigma$-algebra generated by a countable set of subsets of $\Omega$

Definition 5. If $\left(\Omega_{1}, \Sigma_{1}\right)$ and $\left(\Omega_{2}, \Sigma_{2}\right)$ are measurable spaces, the product of the $\sigma$-algebras $\Sigma_{1}$ and $\Sigma_{2}$ is the $\sigma$-algebra $\Sigma_{1} \otimes \Sigma_{2}$ on $\Omega_{1} \times \Omega_{2}$ defined as $\Sigma_{1} \otimes$ $\Sigma_{2}=\sigma\left(\left\{\left(A_{1} \times A_{2} \mid A_{1} \in \Sigma_{1}, A_{2} \in \Sigma_{2}\right\}\right)\right.$. This definition extends naturally to arbitrary finite products of measures.

Definition 6. $A$ Borel $\sigma$-algebra $\mathcal{R}$ on $\mathbb{R}$ is the $\sigma$-algebra generated by the set of open intervals $(a, \infty)$ for $a \in \mathbb{R}$. A Borel $\sigma$-algebra $\mathcal{R}_{n}$ on $\mathbb{R}^{n}$ is the $n$-fold product of $\mathcal{R}$.

\section{Measures}

Definition 7. A measure on the measurable space $(\Omega, \Sigma)$ is a function $\mu: \Sigma \mapsto$ $\overline{\mathbb{R}}_{+}$such that $\mu(\emptyset)=0$ and for any collection of pairwise disjoint sets $A_{1}, A_{2}, \ldots$, $\mu\left(\bigcup_{i \in \mathbb{N}} A_{i}\right)=\sum_{i \in \mathbb{N}} \mu\left(A_{i}\right)$ (i.e. $\mu$ is countably additive).

Definition 8. $A$ product $\mu_{1} \otimes \mu_{2}$ of measures $\mu_{1}$ and $\mu_{2}$ on $\left(\Omega_{1}, \Sigma_{1}\right)$ and $\left(\Omega_{2}, \Sigma_{2}\right)$, respectively, is the unique measure on $\left(\Omega_{1} \times \Omega_{2}, \Sigma_{1} \times \Sigma_{2}\right)$ which satisfies $\left(\mu_{1} \otimes \mu_{2}\right)\left(A_{1} \times A_{2}\right)=\mu_{1}\left(A_{1}\right) \mu_{2}\left(A_{2}\right)$ for all $A_{1} \in \Sigma_{1}, A_{2} \in \Sigma_{2}$. This definition extends naturally to finite products of higher dimensions.

Definition 9. The Lebesgue measure on $(\mathbb{R}, \mathcal{R})$ is the unique measure $\mu_{L}$ which satisfies $\mu_{L}([a, b])=b-a$ for all $a, b \in \mathbb{R}$ such that $b \geq a$. The Lebesgue measure on $\left(\mathbb{R}^{n}, \mathcal{R}_{n}\right)$ is the $n$-fold product of $\mu_{L}$.

Definition 10. A probability measure on $(\Omega, \Sigma)$ is a measure $\mu$ such that $\mu(\Omega)=1$. A subprobability measure on $(\Omega, \Sigma)$ is a measure $\mu$ with $\mu(\Omega) \leq 1$.

Definition 11. A measure $\mu$ on $(\Omega, \Sigma)$ is $\sigma$-finite if there exists a sequence of sets $A_{i} \in \Sigma$ such that $A_{i} \subseteq A_{i+1}$ for all $i$ and $\mu\left(A_{i}\right)<\infty$ and $\Omega=\bigcup_{i \in \mathbb{N}} A_{i}$. 


\section{Measurable functions and integrals}

Definition 12. A function $f$ between measurable spaces $\left(\Omega_{1}, \Sigma_{1}\right)$ and $\left(\Omega_{2}, \Sigma_{2}\right)$ is measurable $\Sigma_{1} / \Sigma_{2}$ if for all $B \in \Sigma_{2}, f^{-1}(B) \in \Sigma_{1}$. If the $\sigma$-algebras $\Sigma_{1}$ and $\Sigma_{2}$ are clear from the context, we will simply call $f$ measurable.

Definition 13. For a measurable space $(\Omega, \Sigma)$, a simple function $g: \Omega \rightarrow \mathbb{R}_{+}$ is a measurable $\Sigma / \mathcal{R}$ function with a finite image set, which can be expressed as $g(x)=\sum_{i=1}^{n} \alpha_{i}\left[x \in A_{i}\right]$, where $A_{i}=f^{-1}\left(\alpha_{1}\right)$. The Lebesgue integral of a simple function $g(x)=\sum_{i=1}^{n} \alpha_{i}\left[x \in A_{i}\right]$ with respect to a measure $\mu$ on $(\Omega, \Sigma)$ is defined as:

$$
\int g(x) \mu(d x)=\sum_{i=1}^{n} \alpha_{i} \mu\left(A_{i}\right)
$$

The Lebesgue integral of any measurable function $f$ is then defined as the limit of integrals of simple functions pointwise smaller than $f$ :

$$
\int f(x) \mu(d x)=\sup \left\{\int g(x) \mu(d x) \mid g \text { simple, } g \leq f\right\}
$$

Theorem 3 (Beppo Levi). Let $f_{i}: X \rightarrow \overline{\mathbb{R}}_{+}$be a (pointwise) non-decreasing sequence of positive measurable functions and let $f=\lim _{n \rightarrow \infty} \int f_{i}$ be the pointwise limit of the sequence. Then $f$ is measurable and

$$
\int f d \mu=\lim _{n \rightarrow \infty} f_{n} d \mu
$$

The same holds for non-increasing sequences, provided that $\int f_{0} d \mu<\infty$.

Note that the limit and supremum of a non-decreasing sequence coincide. limit and infimum of a non-increasing sequence also coincide.

\section{Metric and topological spaces}

Definition 14. A metric on a set $\Omega$ is a function $d: \Omega \times \Omega \rightarrow \overline{\mathbb{R}}_{+}$such that $d(x, x)=0$ and $d(x, y)+d(y, z) \geq d(x, z)$ for all $x, y, z \in \Omega$. The pair $(\Omega, d)$ is called a metric space.

Definition 15. If $(\Omega, d)$ is a metric space, $A \subseteq \Omega$ is open if every element $x \in A$ has a neighbourhood which is completely enclosed in $A$, i.e. there exists $\epsilon>0$ such that $\{y \in \Omega \mid d(x, y)<\epsilon\} \subseteq A$.

Definition 16. If $\left(\Omega_{1}, d_{1}\right)$ and $\left(\Omega_{2}, d_{2}\right)$ are metric spaces, then a product of $\left(\Omega_{1}, d_{1}\right)$ and $\left(\Omega_{2}, d_{2}\right)$ is the metric space $\left(\Omega_{1} \times \Omega_{2}, d_{12}\right)$, where $d_{12}$ is the Manhattan product of metrics $d_{1}$ and $d_{2}$, defined as

$$
d_{12}\left(\left(x_{1}, y_{1}\right),\left(x_{2}, y_{2}\right)\right)=d_{1}\left(x_{1}, y_{1}\right)+d_{2}\left(x_{2}, y_{2}\right) .
$$

This definition naturally extends to finite products of higher dimensions. 
A product of topological spaces can also be defined using the standard Euclidean product metric $d_{12}\left(\left(x_{1}, y_{1}\right),\left(x_{2}, y_{2}\right)\right)=\sqrt{d_{1}\left(x_{1}, y_{1}\right)^{2}+d_{2}\left(x_{2}, y_{2}\right)^{2}}$, both metrics induce the same topologies. We use Manhattan products as they are easier to work with.

Definition 17. $A$ topology on a set $\Omega$ is a set $\mathcal{O}$ of subsets of $\Omega$ such that

$-\emptyset \in \mathcal{O}$

$-\Omega \in \mathcal{O}$

- For all $O_{1}, \ldots, O_{n} \in \mathcal{O}, O_{1} \cap O_{2} \cap \cdots \cap O_{n} \in \mathcal{O}$

- If $O_{i} \in \mathcal{O}$ for all $i \in \mathbb{N}$, then $\bigcup_{n \in \mathbb{N}} O_{i} \in \mathcal{O}$.

The pair $(\Omega, \mathcal{O})$ is called a topological space and the elements of the topology $\mathcal{O}$ are called open sets.

Definition 18. If $\left(\Omega_{1}, \mathcal{O}_{1}\right)$ and $\left(\Omega_{2}, \mathcal{O}_{2}\right)$ are topological spaces, then a product of $\left(\Omega_{1}, d_{1}\right)$ and $\left(\Omega_{2}, d_{2}\right)$ is the metric space $\left(\Omega_{1} \times \Omega_{2}, \mathcal{O}_{1} \times \mathcal{O}_{2}\right)$, where the product of topologies $\mathcal{O}_{1} \times \mathcal{O}_{2}$ is the smallest topology on $\Omega_{1} \times \Omega_{2}$ which makes both left and right projections continuous. This definition naturally extends to final products of higher dimensions.

Definition 19. A function $f$ between metric spaces $\left(\Omega_{1}, d_{1}\right)$ and $\left(\Omega_{2}, d_{2}\right)$ is continuous if for every $x \in \Omega_{1}$ and $\epsilon>0$, there exists $\delta$ such that for all $y \in \Omega_{1}$, if $d_{1}(x, y)<\epsilon$, then $d_{2}(f(x), f(y))<\delta$.

Definition 20. A function $f$ between topological spaces $\left(\Omega_{1}, \mathcal{O}_{1}\right)$ and $\left(\Omega_{2}, \mathcal{O}_{2}\right)$ is continuous if for every open set $O \in \mathcal{O}_{2}, f^{-1}(O) \in \mathcal{O}_{1}$.

\section{From metric to measurable spaces}

Definition 21. A topology on $\Omega$ induced by a metric $d$ is the smallest topology which contains all open sets of the metric space $(\Omega, d)$.

Definition 22. The Borel $\sigma$-algebra $\mathcal{B}(\Omega, \mathcal{O})$ is the $\sigma$-algebra generated by a topology $\mathcal{O}$ on $\Omega$.

Definition 23. We call the Borel $\sigma$-algebra on $\Omega$ generated by the topology induced by the metric $d$ the $\sigma$-algebra induced by $d$. We denote such a $\sigma$ algebra by $\mathcal{B}(\Omega, d)$.

The following lemmas are well-established results:

Lemma 11. If $\mathcal{O}_{1}$ and $\mathcal{O}_{2}$ are, respectively, topologies on $\Omega_{1}$ and $\Omega_{2}$ induced by metrics $r_{1}$ and $r_{2}$, and a function $f$ between the metric spaces $\left(\Omega_{1}, d_{1}\right)$ and $\left(\Omega_{2}, d_{2}\right)$ is continuous, then $f$ is also continuous as a function between topological spaces $\left(\Omega_{1}, \mathcal{O}_{1}\right)$ and $\left(\Omega_{2}, \mathcal{O}_{2}\right)$.

Lemma 12. If $f$ is a continuous function between topological spaces $\left(\Omega_{1}, \mathcal{O}_{1}\right)$ and $\left(\Omega_{2}, \mathcal{O}_{2}\right)$ and $\Sigma_{1}$ and $\Sigma_{2}$ are the Borel $\sigma$-algebras on, respectively, $\Omega_{1}$ and $\Omega_{2}$ generated by topologies $\mathcal{O}_{1}$ and $\mathcal{O}_{2}$, then the function $f$ is measurable. 
Corollary 2. If $\left(\Omega_{1}, d_{1}\right)$ and $\left(\Omega_{2}, d_{2}\right)$ are metric spaces and $f$ is a continuous function from $\Omega_{1}$ to $\Omega_{2}$, then $f$ is measurable $\mathcal{B}\left(\Omega_{1}, d_{1}\right) / \mathcal{B}\left(\Omega_{2}, d_{2}\right)$

Lemma 13. If $\left(\Omega_{1}, d_{1}\right)$ and $\left(\Omega_{2}, d_{2}\right)$ are separable metric spaces, then for the Manhattan product $d_{12}$ of metrics $d_{1}$ and $d_{2}$

$$
\mathcal{B}\left(\Omega_{1} \times \Omega_{2}, d_{12}\right)=\mathcal{B}\left(\Omega_{1}, d_{1}\right) \times \mathcal{B}\left(\Omega_{2}, d_{2}\right)
$$

Corollary 3. If $\left(\Omega_{1}, d_{1}\right),\left(\Omega_{2}, d_{2}\right),\left(\Omega_{3}, d_{3}\right)$ and $\left(\Omega_{4}, d_{4}\right)$ are separable metric spaces and $f$ is a continuous function from $\Omega_{1} \times \Omega_{2}$ to $\Omega_{3} \times \Omega_{4}$ (with respect to corresponding product metrics) then $f$ is measurable $\mathcal{B}\left(\Omega_{1}, d_{1}\right) \times$ $\mathcal{B}\left(\Omega_{2}, d_{2}\right) / \mathcal{B}\left(\Omega_{3}, d_{3}\right) \times \mathcal{B}\left(\Omega_{4}, d_{4}\right)$.

All the above results extend naturally to arbitrary finite products.

\section{B Basics of domain theory}

This section includes some basic definitions from domain theory which are required to understand the paper. For readers wanting a more complete, tutorialstyle introduction, there are many resources available, including 17] and [1].

Please note that we use the notions of $\omega$-complete partial order and $\omega$ continuity, defined in terms of countable sequences of increasing values $(\omega$ chains), rather than the more general notions of complete partial order (requiring existence of suprema of directed sets) and continuity (requiring the given function to preserve suprema of all subsets of the domain). While $\omega$-completeness and $\omega$-continuity are technically weaker than completeness and continuity, respectively, they are sufficient for our purposes, as they allow applying the Kleene Fixpoint Theorem.

Definition 24 (Partially-ordered set). A partially-ordered set is a pair ( $D$, ) of set $D$ and relation $\sqsubseteq$ such that:

- For each $a \in D, a \sqsubseteq a$ (reflexiveness)

- For each $a, b, c \in D$, if $a \sqsubseteq b$ and $b \sqsubseteq c$, then $a \sqsubseteq c$ (transitivity)

- For each $a, b \in D$, if $a \sqsubseteq b$ and $b \sqsubseteq a$, then $a=b$ (antisymmetry)

Definition 25 ( $\omega$-chain and its supremum). A $\omega$-chain in a partially-ordered set $(D, \sqsubseteq)$ is an infinite sequence $d_{0}, d_{1}, d_{2}, \ldots$ such that for all $i, d_{i} \in D$ and $d_{i} \sqsubseteq d_{i+1}$. The supremum $\sup _{i} d_{i}$ of a chain $d_{0}, d_{1}, d_{2}, \ldots$ is the supremum of the set $\left\{d_{0}, d_{1}, d_{2}, \ldots\right\}$ of elements of the chain.

Definition 26 ( $\omega$-complete partial order). $A \omega$-complete partial order ( $\omega$ cpo) is a partial order $(D, \sqsubseteq)$ such that for each $\omega$-chain $d_{0}, d_{1}, d_{2}, \ldots$ in $(D, \sqsubseteq)$, the supremum $\sup _{i} d_{i}$ exists in $D$.

Definition 27 (Monotone function). A function $f: D \rightarrow D^{\prime}$ between $\omega$-cpos $(D, \sqsubseteq)$ and $\left(D^{\prime}, \sqsubseteq^{\prime}\right)$ is monotone if $f(d) \sqsubseteq^{\prime} f\left(d^{\prime}\right)$ for each $d, d^{\prime} \in D$ such that $d \sqsubseteq d^{\prime}$. 
Definition 28 ( $\omega$-continuous function). A function $f: D \rightarrow D^{\prime}$ between $\omega$ cpos $(D, \sqsubseteq)$ and $\left(D^{\prime}, \sqsubseteq^{\prime}\right)$ is $\omega$-continuous if it is monotone and for each $\omega$-chain $d_{0}, d_{1}, d_{2}, \ldots$ in $(D, \sqsubseteq), f\left(\sup _{i} d_{i}\right)=\sup _{i} f\left(d_{i}\right)$.

Note that in the definition above, the requirement that $f$ is monotone ensures that $f\left(d_{0}\right), f\left(d_{1}\right), f\left(d_{2}\right), \ldots$ is a $\omega$-chain.

Definition 29 (Least fixpoint). Let $(D, \sqsubseteq)$ be a $\omega$-cpo and $f: D \rightarrow D$ a function on $(D, \sqsubseteq)$. A fixpoint of $f$ is an element $d \in D$ such that $f(d)=d$. A least fixpoint of $f$ is a fixpoint $d_{0}$ of $f$ such that for all other fixpoints $d$ of $f$, $d_{0} \sqsubseteq d$.

Theorem 4 (Kleene Fixpoint Theorem). Let $(D, \sqsubseteq)$ be $a \omega$-cpo and $f: D \rightarrow$ $D$ a $\omega$-continuous function. Then $f$ has a least fixpoint, which is the supremum of the chain $\perp, f(\perp), f(f(\perp)), \ldots$, that is, $\sup _{i} f^{i}(\perp)$.

\section{Proofs for the wp and wlp semantics}

In order to prove that $w \llbracket \llbracket C \rrbracket(f)$ is measurable for all $f$, we first need to prove that the state update $\lambda(x, \sigma, E) . \sigma[x \mapsto \sigma(E)]$ is measurable. Since states are a new structure, not discussed in the proofs of measurability in [36], we present the proof in more detail than other measurability proofs in this paper.

We define a metric $d_{\mathcal{N}}$ on variables as $d_{\mathcal{N}}(x, x)=0$ and $d_{\mathcal{N}}(x, y)=\infty$ for $x \neq y$. The metric space $\left(\mathcal{N}, d_{\mathcal{N}}\right)$ induces the usual discrete $\sigma$-algebra on $\mathcal{N}$.

Lemma 14. The update function $h: \mathcal{N} \times \Omega_{\sigma} \times(\mathbb{R} \uplus \mathbb{Z}) \rightarrow \Omega_{\sigma}$ defined by $h(x, \sigma, v)=$ $\sigma[x \mapsto v]$, is measurable.

Proof. We prove that this function is continuous, which implies measurability. Take $x_{1}, x_{2} \in \mathcal{N}, \sigma_{1}, \sigma_{2} \in \Omega_{\sigma}$ and $V_{1}, V_{2} \in \mathbb{R} \uplus \mathbb{Z}$. If $\operatorname{dom}\left(\sigma_{1}\right) \neq \operatorname{dom}\left(\sigma_{2}\right)$ then $d_{\sigma}\left(\sigma_{1}, \sigma_{2}\right)=\infty$, so trivially $d_{\sigma}\left(h\left(x_{1}, \sigma_{1}, V_{1}\right), h\left(x_{2}, \sigma_{2}, V_{2}\right)\right) \leq d_{\mathcal{N}}\left(x_{1}, x_{2}\right)+$ $d_{\sigma}\left(\sigma_{1}, \sigma_{2}\right)+d_{T}\left(V_{1}, V_{2}\right)=\infty$. The same holds when $x_{1} \neq x_{2}$ (which implies $\left.d_{\mathcal{N}}\left(x_{1}, x_{2}\right)=\infty\right)$. The inequality also immediately holds if $V_{1} \in \mathbb{R}$ and $V_{2} \in \mathbb{Z}$ (or vice versa), because then $d_{T}\left(V_{1}, V_{2}\right)=\infty$.

Now, suppose that $x_{1}=x_{2}=x, \operatorname{dom}\left(\sigma_{1}\right)=\operatorname{dom}\left(\sigma_{2}\right)=\left\{y_{1}, \ldots, y_{n}\right\}$ and either $V_{1}, V_{2} \in \mathbb{R}$ or $V_{1}, V_{2} \in \mathbb{Z}$. Now, if $x=y_{k}$ for some $k$, then

$$
\begin{aligned}
d_{\sigma}\left(h\left(x, \sigma_{1}, V_{1}\right), h\left(x, \sigma_{2}, V_{2}\right)\right) & =\sum_{i \in 1 . . n, i \neq k} d_{T}\left(\sigma_{1}\left(y_{i}\right), \sigma_{2}\left(y_{i}\right)\right)+d_{T}\left(V_{1}, V_{2}\right) \\
& \leq \sum_{i \in 1 . . n} d_{T}\left(\sigma_{1}\left(y_{i}\right), \sigma_{2}\left(y_{i}\right)\right)+d_{T}\left(V_{1}, V_{2}\right) \\
& =d_{\sigma}\left(\sigma_{1}, \sigma_{2}\right)+d_{T}\left(V_{1}, V_{2}\right)+d_{\mathcal{N}}(x, x)
\end{aligned}
$$

If $x \neq x_{k}$ for any $k$, we simply have:

$$
\begin{aligned}
d_{\sigma}\left(h\left(x, \sigma_{1}, V_{1}\right), h\left(x, \sigma_{2}, V_{2}\right)\right) & =\sum_{i \in 1 . . n} d_{T}\left(\sigma_{1}\left(y_{i}\right), \sigma_{2}\left(y_{i}\right)\right)+d_{T}\left(V_{1}, V_{2}\right) \\
& =d_{\sigma}\left(\sigma_{1}, \sigma_{2}\right)+d_{T}\left(V_{1}, V_{2}\right)+d_{\mathcal{N}}(x, x)
\end{aligned}
$$

Thus, $h_{x}$ is continuous, and so measurable. 
Restatement of Lemma 3 For every program $C$, the function wp $\llbracket C \rrbracket(\cdot)$ is $\omega$-continuous. Moreover, for every measurable $f: \Omega_{\sigma} \rightarrow \overline{\mathbb{R}}_{+}$, wp $\llbracket C \rrbracket(f)(\cdot)$ is measurable.

Proof (of Lemma 3). By induction on the structure of $C$. The continuity part of the proof is largely similar to the proof of the analogous property in [13], with additional care needed because of the use of Lebesgue integration. We need to show that for any $C$ and any $\omega$-chain $f_{1} \leq f_{2} \leq f_{3} \ldots$, wp $\llbracket C \rrbracket\left(\sup _{i} f_{i}\right)=$ $\sup _{i} \operatorname{wp} \llbracket C \rrbracket\left(f_{i}\right)$ and that $\operatorname{wp} \llbracket C \rrbracket(f)$ is measurable for any measurable $f$.

- Case $C=x: \approx U$ :

- Continuity:

$$
\begin{aligned}
\operatorname{wp} \llbracket C \rrbracket\left(\sup _{i} f_{i}\right) & =\lambda \sigma \cdot \int_{[0,1]}\left(\sup _{i} f_{i}\right)(\sigma[x \mapsto v]) \mu_{L}(d v) \\
\text { (by Beppo Levi's theorem) } & =\lambda \sigma \cdot \sup _{i} \int_{[0,1]} f_{i}(\sigma[x \mapsto v]) \mu_{L}(d v) \\
\text { (sup taken wrt pointwise ordering) } & =\sup _{i} \lambda \sigma \cdot \int_{[0,1]} f_{i}(\sigma[x \mapsto v]) \mu_{L}(d v) \\
& =\sup _{i} \operatorname{wp} \llbracket C \rrbracket\left(f_{i}\right)
\end{aligned}
$$

- Measurability:

We have

$$
\mathrm{wp} \llbracket C \rrbracket(f)=\lambda \sigma \cdot \int_{[0,1]} g(x, \sigma, v) \mu_{L}(d v)
$$

where $g(x, \sigma, v)=f(\sigma[x \mapsto v])$. Now, take $h(x, \sigma, v)=\sigma[x \mapsto v]$. Then $g=f \circ h$. We know that substitutions are measurable (Lemnma 14), so $h$ is measurable. This means that $g$ is measurable, as it is a composition of measurable functions. Thus, by the Fubini-Tonelli theorem, $\lambda \sigma . \int_{[0,1]} g(x, \sigma, v) \mu_{L}(d v)$ is measurable, so wp $\llbracket C \rrbracket(f)$ is measurable.

- Case $C=\operatorname{score}(E)$ :

- Continuity:

$$
\begin{aligned}
\operatorname{wp} \llbracket C \rrbracket\left(\sup _{i} f_{i}\right) & =\lambda \sigma \cdot[\sigma(E) \in(0,1]] \sigma(E) \cdot\left(\sup _{i} f_{i}\right)(\sigma) \\
\text { (multiplying by a constant preserves sup) } & =\lambda \sigma \cdot \sup _{i}\left([\sigma(E) \in(0,1]] \sigma(E) \cdot f_{i}(\sigma)\right) \\
\text { (sup taken wrt pointwise ordering) } & =\sup _{i} \lambda \sigma \cdot[\sigma(E) \in(0,1]] \sigma(E) \cdot f_{i}(\sigma) \\
& =\sup _{i} \operatorname{wp} \llbracket C \rrbracket\left(f_{i}\right)
\end{aligned}
$$

- Measurability:

We have wp $\llbracket C \rrbracket(f)=\lambda \sigma$. $[\sigma(E) \in(0,1]] \sigma(E) \cdot f(\sigma)$. The substitution $\sigma(E)$ is measurable by assumption (as a function of $\sigma$ ). Meanwhile, $[\sigma(E) \in(0,1]]$ is a composition of the measurable function $\sigma(E)$ and the indicator function of the measurable set $(0,1]$, which is obviously measurable. Finally, $f$ is measurable by assumption, so the pointwise product of these three functions is measurable. 
- Case $C=$ observe $(\phi)$ :

- Continuity:

$$
\begin{aligned}
\operatorname{wp} \llbracket C \rrbracket\left(\sup _{i} f_{i}\right) & =\lambda \sigma \cdot[\sigma(\phi)]\left(\sup _{i} f_{i}\right)(\sigma) \\
\text { (multiplying by a constant preserves sup }) & =\lambda \sigma \cdot \sup _{i}\left([\sigma(\phi)] f_{i}(\sigma)\right) \\
(\text { sup taken wrt pointwise ordering) } & =\sup _{i} \lambda \sigma \cdot[\sigma(\phi)] f_{i}(\sigma) \\
& =\sup _{i} \operatorname{wp} \llbracket C \rrbracket\left(f_{i}\right)
\end{aligned}
$$

\section{- Measurability:}

We have wp $\llbracket C \rrbracket(f)=\lambda \sigma \cdot[\sigma(\phi)] f(\sigma)$. The function $\sigma \cdot[\sigma(\phi)]$ is measurable by assumption (we only allow measurable predicates in the language), and $f$ is measurable by assumption of the lemma, hence their pointwise product is measurable.

- Case $C=(x:=E)$ :

- Continuity:

$$
\begin{aligned}
\operatorname{wp} \llbracket C \rrbracket\left(\sup _{i} f_{i}\right) & =\lambda \sigma \cdot\left(\sup _{i} f_{i}\right)(\sigma[x \mapsto \sigma(E)]) \\
\text { (sup taken wrt pointwise ordering) } & =\lambda \sigma \cdot \sup _{i} f_{i}(\sigma[x \mapsto \sigma(E)]) \\
\text { (sup taken wrt pointwise ordering) } & =\sup _{i} \lambda \sigma \cdot f_{i}(\sigma[x \mapsto \sigma(E)]) \\
& =\sup _{i} \operatorname{wp} \llbracket C \rrbracket\left(f_{i}\right)
\end{aligned}
$$

\section{- Measurability:}

We have wp $\llbracket C \rrbracket(f)=\lambda \sigma . f(\sigma[x \mapsto \sigma(E)])$. This can be represented as a composition of functions $\lambda \sigma . f \circ F_{2} \circ F_{1}(\sigma)$, where $F_{1}(\sigma)=(\sigma, \sigma(E))$ and $F_{2}(\sigma, V)=\sigma[x \mapsto V]$. The function $F_{1}$ is measurable, because the identity function $\lambda \sigma . \sigma$ is trivially measurable, and $\lambda \sigma . \sigma(E)$ is measurable by assumption, so both components of $F_{1}$ are measurable. The function $F_{2}$ is measurable by Lemma 14 Hence, wp $\llbracket C \rrbracket(f)$ is measurable as a composition of measurable functions.

- Case $C=$ while $(\phi)\left\{C^{\prime}\right\}$ :

- Continuity: We have:

$$
\begin{aligned}
\operatorname{wp} \llbracket C \rrbracket\left(\sup _{i} f_{i}\right) & =\operatorname{wp} \llbracket \operatorname{while}(\phi)\left\{C^{\prime}\right\} \rrbracket\left(\sup _{i} f_{i}\right) \\
& =\operatorname{lfp} X .[\neg \phi]\left(\sup _{i} f_{i}\right)+[\phi] \operatorname{wp} \llbracket C^{\prime} \rrbracket(X)
\end{aligned}
$$

Take $\Phi_{f}(X)=[\neg \phi] f+[\phi] \mathrm{wp} \llbracket C^{\prime} \rrbracket(X)$. By induction hypothesis, wp $\llbracket C^{\prime} \rrbracket(\cdot)$ is continuous, so $\Phi_{f}(\cdot)$ is continuous for all $f: \Omega_{\sigma} \rightarrow \overline{\mathbb{R}}_{+}$. Moreover, it can be easily checked that for any $X, f \mapsto \Phi_{f}(X)$ is continuous as a function of $f$ (which means that $f \mapsto \Phi_{f}$ is continuous). Thus,

$$
\operatorname{wp} \llbracket C \rrbracket\left(\sup _{i} f_{i}\right)=\sup _{n} \Phi_{\sup _{i} f_{i}}^{n}(0)=\sup _{n}\left(\sup _{i} \Phi_{f_{i}}\right)^{n}(0)
$$


By Theorem 2.1.19.2 from [1], the function $\Phi \mapsto \sup _{n} \Phi^{n}(0)$ is continuous. If $f_{1}, f_{2}, \ldots$ is an increasing chain, then $\Phi_{f_{1}}, \Phi_{f_{2}}, \ldots$ is also an increasing chain (because $\Phi_{f}$ is monotone in $f$ ). Thus, $\sup _{n}\left(\sup _{i} \Phi_{f_{i}}\right)^{n}(0)=$ $\sup _{i}\left(\sup _{n} \Phi_{f_{i}}^{n}(0)\right)=\sup _{i} \operatorname{wp} \llbracket C \rrbracket\left(f_{i}\right)$, as required.

- Measurability:

The function $\Phi_{f}(X)=[\neg \phi](f)+[\phi] w p \llbracket C^{\prime} \rrbracket(X)$ is continuous for all measurable $f$ by the induction hypothesis, so by the fixpoint theorem lfp $X . \Phi_{f}(X)$ exists in the domain of measurable functions.

- Case $C=C_{1} ; C_{2}$ :

- Continuity:

We have :

$$
\operatorname{wp} \llbracket C \rrbracket\left(\sup _{i} f_{i}\right)=\operatorname{wp} \llbracket C_{1} \rrbracket\left(\operatorname{wp} \llbracket C_{2} \rrbracket\left(\sup _{i} f_{i}\right)\right)
$$

By induction hypothesis, $\operatorname{wp} \llbracket C_{2} \rrbracket\left(\sup _{i} f_{i}\right)=\sup _{i} \operatorname{wp} \llbracket C_{2} \rrbracket\left(f_{i}\right)$. The induction hypothesis also states that $\mathrm{wp} \llbracket C_{2} \rrbracket\left(f_{i}\right)$ is measurable for all measurable $f_{i}$, which also means that $\sup _{i} \operatorname{wp} \llbracket C_{2} \rrbracket\left(f_{i}\right)$ is measurable. Hence, $\operatorname{wp} \llbracket C_{1} \rrbracket\left(\sup _{i} \operatorname{wp} \llbracket C_{2} \rrbracket\left(f_{i}\right)\right)$ is well-defined. By applying the induction hypothesis again, we get $\operatorname{wp} \llbracket C_{1} \rrbracket\left(\sup _{i} \operatorname{wp} \llbracket C_{2} \rrbracket\left(f_{i}\right)\right)=\sup _{i} \operatorname{wp} \llbracket C_{1} \rrbracket\left(\operatorname{wp} \llbracket C_{2} \rrbracket\left(f_{i}\right)\right)$, as required.

- Measurability:

By induction hypothesis, wp $\llbracket C_{2} \rrbracket(f)$ is measurable, and so wp $\llbracket C_{1} \rrbracket\left(\right.$ wp $\left.\llbracket C_{2} \rrbracket(f)\right)$ is also measurable by induction hypothesis.

- The other cases are straightforward.

\section{Proofs for the operational semantics}

\section{D.1 Properties of the operational semantics}

This section consists of proofs of properties of the operational semantics which are needed to prove Proposition 1 .

Basic properites We begin by stating two basic properties: that reduction is deterministic and that the weight always stays positive.

Lemma 15 (Evaluation is deterministic). For any configuration $\kappa$, if $\kappa \vdash \kappa^{\prime}$ and $\kappa \vdash \kappa^{\prime \prime}$, then $\kappa^{\prime}=\kappa^{\prime \prime}$.

Lemma 16. If $\kappa \vdash \kappa^{\prime}$ and weight $(\kappa)>0$, then weight $\left(\kappa^{\prime}\right)>0$.

Invariance of reduction relation The functions $\mathbf{O}_{C}^{\sigma}$ and $\mathbf{S} \mathbf{C}_{C}^{\sigma}$ are defined in terms of reduction chains which start at configurations with $K=$ [], $n=0$ and $w=1$. However, in order to reason about evaluation of compositions of terms, we need to deal with reduction sequences starting at intermediate configurations, where this property does not hold. The following lemmas show that the reduction 
relation is preserved by modifying the initial and final step count, weight and continuation.

Proving invariance of the semantics under step count and weight change is straightforward:

Lemma 17. If $\left\langle\theta, C, K, \sigma, \theta_{K}, n, w\right\rangle \vdash^{*}\left\langle\theta^{\prime}, C^{\prime}, K^{\prime}, \sigma^{\prime}, \theta_{K}^{\prime}, n+n^{\prime}, w^{\prime}\right\rangle$, then for all $w^{\prime \prime}>0$ and integer $n^{\prime \prime} \geq-n,\left\langle\theta, C, K, \sigma, \theta_{K}, n+n^{\prime \prime}, w^{\prime \prime} w\right\rangle \vdash^{*}\left\langle\theta^{\prime}, C^{\prime}, K^{\prime}, \sigma^{\prime}\right.$, $\left.\theta_{K}^{\prime}, n+n^{\prime \prime}+n^{\prime}, w^{\prime \prime} w^{\prime}\right\rangle$.

Proof. Simple induction on $n^{\prime}$.

The rest of this section shows that the semantics is also preserved by extending the initial continuation. In the following lemmas, we write $K @ K^{\prime}$ for the concatenation of two continuations $K$ and $K^{\prime}$ (recall that a continuation is a list of expressions).

Lemma 18. - If $\left\langle\theta, C, K^{\prime}, \sigma, \theta_{K}, n, w\right\rangle \vdash\left\langle\theta^{\prime}, C^{\prime}, K^{\prime \prime}, \sigma^{\prime}, \theta_{K}^{\prime}, n+1, w^{\prime}\right\rangle$ and $\sigma^{\prime} \neq$ 々 and $\left(C, K^{\prime}\right) \neq(\downarrow,[])$, then $\left\langle\theta, C, K^{\prime} @ K, \sigma, \theta_{K}, n, w\right\rangle \vdash\left\langle\theta^{\prime}, C^{\prime}, K^{\prime \prime} @ K, \sigma^{\prime}\right.$, $\left.\theta_{K}^{\prime}, n+1, w^{\prime}\right\rangle$.

- If $\left\langle\theta, C, K^{\prime}, \sigma, \theta_{K}, n, w\right\rangle \vdash\left\langle\theta^{\prime}, C^{\prime}, K^{\prime \prime}, \downarrow, \theta_{K}^{\prime}, n+1, w^{\prime}\right\rangle$ then $\left\langle\theta, C, K^{\prime} @ K, \sigma\right.$,

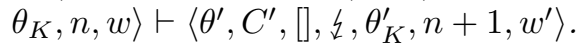

Proof. By inspection of the reduction rules.

Lemma 19. If $\left\langle\theta, C, K, \sigma, \theta_{K}, n, w\right\rangle \vdash^{*}\left\langle\theta^{\prime}, \downarrow,[], \sigma^{\prime}, \theta_{K}^{\prime}, n+n^{\prime}, w^{\prime}\right\rangle$, then there exists a unique $\hat{n} \leq n^{\prime}$ such that $\left\langle\theta, C, K, \sigma, \theta_{K}, n, w\right\rangle \vdash_{\min }^{*}\left\langle\theta^{\prime}, \downarrow,[], \sigma^{\prime}, \theta_{K}^{\prime}, n+\hat{n}\right.$, $\left.w^{\prime}\right\rangle$

Proof. Obvious.

Lemma 20. If $\left\langle\theta, C, K, \sigma, \theta_{K}, n, w\right\rangle \vdash^{*}\left\langle\theta^{\prime}, C^{\prime}, K^{\prime}, \sigma^{\prime}, \theta_{K}^{\prime}, n+n^{\prime}, w^{\prime}\right\rangle$ and $\left(C^{\prime}, K^{\prime}\right) \neq$ $(\downarrow,[])$ and $\sigma^{\prime} \neq \downarrow$, then for all $K^{\prime \prime},\left\langle\theta, C, K @ K^{\prime \prime}, \sigma, \theta_{K}, n, w\right\rangle \vdash^{*}\left\langle\theta^{\prime}, C^{\prime}, K^{\prime} @ K^{\prime \prime}\right.$, $\left.\sigma^{\prime}, \theta_{K}^{\prime}, n+n^{\prime}, w^{\prime}\right\rangle$.

Proof. By induction on $n^{\prime}$ :

- Base case: $n^{\prime}=0$ : trivial

- Induction step: Let $n^{\prime}>0$. Then we have $\left\langle\theta, C, K, \sigma, \theta_{K}, n, w\right\rangle \vdash\langle\hat{\theta}, \hat{C}, \hat{K}, \hat{\sigma}$, $\left.\hat{\theta_{K}}, n+1, \hat{w}\right\rangle \vdash^{*}\left\langle\theta^{\prime}, C^{\prime}, K^{\prime}, \sigma^{\prime}, \theta_{K}^{\prime}, n+n^{\prime}, w^{\prime}\right\rangle$. We now need to split on the derivation of $\left\langle\theta, C, K, \sigma, \theta_{K}, n, w\right\rangle \vdash\left\langle\hat{\theta}, \hat{C}, \hat{K}, \hat{\sigma}, \hat{\theta_{K}}, n+1, w\right\rangle$.

- If $\left\langle\theta, C, K, \sigma, \theta_{K}, n, w\right\rangle \vdash\left\langle\hat{\theta}, \hat{C}, \hat{K}, \hat{\sigma}, \hat{\theta_{K}}, n+1, \hat{w}\right\rangle$ was derived with (seq), then $C=C_{1} ; C_{2}, \hat{K}=C_{2}:: K$ and we have $\left\langle\theta, C_{1} ; C_{2}, K, \sigma, \theta_{K}, n\right.$, $w\rangle \vdash\left\langle\pi_{L}(\theta), C_{1}, C_{2}:: K, \sigma, \pi_{L}(\theta):: \theta_{K}, n+1, w\right\rangle \vdash^{*}\left\langle\theta^{\prime}, C^{\prime}, K^{\prime}, \sigma^{\prime}, \theta_{K}^{\prime}\right.$, $\left.n+n^{\prime}, w^{\prime}\right\rangle$.

By (seq), $\left\langle\theta, C_{1} ; C_{2}, K @ K^{\prime \prime}, \sigma, \theta_{K}, n, w\right\rangle \vdash\left\langle\pi_{L}(\theta), C_{1}, C_{2}:: K @ K^{\prime \prime}, \sigma\right.$, $\left.\pi_{L}(\theta):: \theta_{K}, n+1, w\right\rangle$, and by the induction hypothesis, $\left\langle\pi_{L}(\theta), C_{1}, C_{2}::\right.$ $\left.K @ K^{\prime \prime}, \sigma, \pi_{L}(\theta):: \theta_{K}, n+1, \hat{w}\right\rangle \vdash^{*}\left\langle\theta^{\prime}, C^{\prime}, K^{\prime} @ K^{\prime \prime}, \sigma^{\prime}, \theta_{K}^{\prime}, n+n^{\prime}, w^{\prime}\right\rangle$. 
- If $\left\langle\theta, C, K, \sigma, \theta_{K}, n, w\right\rangle \vdash\left\langle\hat{\theta}, \hat{C}, \hat{K}, \hat{\sigma}, \hat{\theta_{K}}, n+1, \hat{w}\right\rangle$ was derived with (pop), then $C=\downarrow$ and $K=C^{\prime}:: K^{\prime \prime \prime}$ and we have $\left\langle\theta, \downarrow, C^{\prime}:: K^{\prime \prime \prime}, \sigma, \theta_{K}, n\right.$, $w\rangle \vdash\left\langle\pi_{L}\left(\theta_{K}\right), C^{\prime}, K^{\prime \prime \prime}, \sigma, \pi_{R}\left(\theta_{K}\right), n+1, w\right\rangle \vdash^{*}\left\langle\theta^{\prime}, C^{\prime}, K^{\prime}, \sigma^{\prime}, \theta_{K}^{\prime}, n+n^{\prime}\right.$, $\left.w^{\prime}\right\rangle$.

By (pop), $\left\langle\theta, \downarrow, C^{\prime}:: K^{\prime \prime \prime} @ K^{\prime \prime}, \sigma, \theta_{K}, n, w\right\rangle \vdash\left\langle\pi_{L}\left(\theta_{K}\right), C^{\prime}, K^{\prime \prime \prime} @ K^{\prime \prime}, \sigma\right.$, $\left.\pi_{R}\left(\theta_{K}\right), n+1, w\right\rangle$, and by induction hypothesis, $\left\langle\pi_{L}\left(\theta_{K}\right), C^{\prime}, K^{\prime \prime \prime} @ K^{\prime \prime}\right.$, $\left.\sigma, \pi_{R}\left(\theta_{K}\right), n+1, w\right\rangle \vdash^{*}\left\langle\theta^{\prime}, C^{\prime}, K^{\prime} @ K^{\prime \prime}, \sigma^{\prime}, \theta_{K}^{\prime}, n+n^{\prime}, w^{\prime}\right\rangle$.

- Otherwise, we have $\hat{K}=K$ and by inspection of the reduction rules, $\left\langle\theta, C, K @ K^{\prime \prime}, \sigma, \theta_{K}, n, w\right\rangle \vdash\left\langle\hat{\theta}, \hat{C}, K @ K^{\prime \prime}, \hat{\sigma}, \hat{\theta_{K}}, n+1, \hat{w}\right\rangle$, so the result follows immediately by applying the induction hypothesis (note that $\left(C^{\prime}, K^{\prime}\right) \neq(\downarrow,[])$ implies that $\left\langle\theta, C, K, \sigma, \theta_{K}, n, w\right\rangle \vdash\left\langle\hat{\theta}, \hat{C}, \hat{K}, \hat{\sigma}, \hat{\theta_{K}}, n+\right.$ $1, \hat{w}\rangle$ is not derived with (final)).

Corollary 4. If $\left\langle\theta, C, K, \sigma, \theta_{K}, n, w\right\rangle \vdash^{*}\left\langle\theta^{\prime}, C^{\prime}, K^{\prime}, \sigma^{\prime}, \theta_{K}^{\prime}, n+n^{\prime}, w^{\prime}\right\rangle$ and $\sigma^{\prime} \neq \downarrow$ and $\left(C^{\prime}, K^{\prime}\right) \neq(\downarrow,[])$, then for all $w^{\prime \prime}>0$, integer $n^{\prime \prime} \geq-n$ and $K^{\prime \prime},\langle\theta, C$, $\left.K @ K^{\prime \prime}, \sigma, \theta_{K}, n+n^{\prime \prime}, w^{\prime \prime} w\right\rangle \vdash^{*}\left\langle\theta^{\prime}, C^{\prime}, K^{\prime} @ K^{\prime \prime}, \sigma^{\prime}, \theta_{K}^{\prime}, n+n^{\prime \prime}+n^{\prime}, w^{\prime \prime} w^{\prime}\right\rangle$.

The reason we added the condition $\left(C^{\prime}, K^{\prime}\right) \neq(\downarrow,[])$ to the premise of Lemma 20 is that in our semantics, a "final" configuration with statement $\downarrow$ and empty continuation reduces to itself (by the (final) rule) infinitely. If we replaced [] with some non-empty continuation $K$, the rule (pop) would be applied instead of (final) and the reduction would be completely different. The statement $\left\langle\theta, C, K, \sigma, \theta_{K}, n, w\right\rangle \vdash^{*}\left\langle\theta^{\prime}, \downarrow,[], \sigma^{\prime}, \theta_{K}^{\prime}, n+n^{\prime}, w^{\prime}\right\rangle$ says nothing about how many times the rule (final) was applied at the end, so we do not know what the final configuration after $n^{\prime}$ steps would be if we appended some continuation $K^{\prime}$ to $K$.

Because of that, we need to treat the case $\left(C^{\prime}, K^{\prime}\right)=(\downarrow$, []) separately. We first introduce some new notation: we write $\left\langle\theta, C, K, \sigma, \theta_{K}, n, w\right\rangle \vdash_{\min }^{*}\left\langle\theta^{\prime}, \downarrow\right.$, [], $\left.\sigma^{\prime}, \theta_{K}^{\prime}, n+n^{\prime}, w^{\prime}\right\rangle$ if $\left\langle\theta, C, K, \sigma, \theta_{K}, n, w\right\rangle \vdash^{*}\left\langle\theta^{\prime}, \downarrow,[], \sigma^{\prime}, \theta_{K}^{\prime}, n+n^{\prime}, w^{\prime}\right\rangle$ and there is no $n^{\prime \prime}<n^{\prime}$ such that $\left\langle\theta, C, K, \sigma, \theta_{K}, n, w\right\rangle \vdash^{*}\left\langle\theta^{\prime \prime}, \downarrow,[], \sigma^{\prime \prime}, \theta_{K}^{\prime \prime}, n+n^{\prime \prime}, w^{\prime \prime}\right\rangle$ (or, equivalently, $\left\langle\theta, C, K, \sigma, \theta_{K}, n, w\right\rangle \vdash^{*}\left\langle\theta^{\prime}, \downarrow\right.$, [], $\left.\sigma^{\prime}, \theta_{K}^{\prime}, n+n^{\prime}, w^{\prime}\right\rangle$ was derived without (final)).

Lemma 21 (Evaluation with continuation). If $\left\langle\theta, C\right.$, [], $\left.\sigma, \theta_{K}, n, w\right\rangle \vdash_{\min }^{*}\left\langle\theta^{\prime}\right.$, $\left.\downarrow,[], \sigma^{\prime}, \theta_{K}^{\prime}, n+n^{\prime}, w^{\prime}\right\rangle$ and $\sigma^{\prime} \neq$ 々, then $\left\langle\theta, C, K, \sigma, \theta_{K}, n, w\right\rangle \vdash^{*}\left\langle\theta^{\prime}, \downarrow, K, \sigma^{\prime}, \theta_{K}^{\prime}\right.$, $\left.n+n^{\prime}, w^{\prime}\right\rangle$.

Proof. We will prove a more general statement:

If $\left\langle\theta, C, K^{\prime}, \sigma, \theta_{K}, n, w\right\rangle \vdash_{\min }^{*}\left\langle\theta^{\prime}, \downarrow,[], \sigma^{\prime}, \theta_{K}^{\prime}, n+n^{\prime}, w^{\prime}\right\rangle$, then $\left\langle\theta, C, K^{\prime} @ K, \sigma\right.$, $\left.\theta_{K}, n, w\right\rangle \vdash^{*}\left\langle\theta^{\prime}, \downarrow, K, \sigma^{\prime}, \theta_{K}^{\prime}, n+n^{\prime}, w^{\prime}\right\rangle$,

by induction on $n^{\prime}$ :

- Base case: $n^{\prime}=0$ : This implies that $C=\downarrow$ and $w^{\prime}=w$ and $K^{\prime}=[]$ and $\theta_{K}^{\prime}=\theta_{K}$, so the result follows trivially.

- Induction step: for $n^{\prime}>0$, we have $\left\langle\theta, C, K^{\prime}, \sigma, \theta_{K}, n, w\right\rangle \vdash\left\langle\hat{\theta}, \hat{C}, \hat{K^{\prime}}, \hat{\sigma}, \hat{\theta_{K}}\right.$, $n+1, \hat{w}\rangle \vdash_{\min }^{*}\left\langle\theta^{\prime}, \downarrow,[], \sigma^{\prime}, \theta_{K}^{\prime}, n+n^{\prime}, w^{\prime}\right\rangle$, where $\left(C, K^{\prime}\right) \neq(\downarrow,[])$, as otherwise the configuration would reduce in 0 steps. 
By Lemma 18, $\left\langle\theta, C, K^{\prime} @ K, \sigma, \theta_{K}, n, w\right\rangle \vdash\left\langle\hat{\theta}, \hat{C}, \hat{K}^{\prime} @ K, \hat{\sigma}, \hat{\theta_{K}}, n+1, \hat{w}\right\rangle$ and by induction hypothesis, $\left\langle\hat{\theta}, \hat{C}, \hat{K}^{\prime} @ K, \hat{\sigma}, \hat{\theta_{K}}, n+1, \hat{w}\right\rangle \vdash^{*}\left\langle\theta^{\prime}, \downarrow, K, \sigma^{\prime}, \theta_{K}^{\prime}\right.$, $\left.n+1+\left(n^{\prime}-1\right), w^{\prime}\right\rangle$, which ends the proof.

Corollary 5. If $\left\langle\theta, C\right.$, [], $\left.\sigma, \theta_{K}, n, w\right\rangle \vdash_{\min }^{*}\left\langle\theta^{\prime}, \downarrow,[], \sigma^{\prime}, \theta_{K}, n+n^{\prime}, w^{\prime}\right\rangle$ and $\sigma^{\prime} \neq \downarrow$, then $\left\langle\theta, C, K, \sigma, \theta_{K}, n, w\right\rangle \vdash^{*}\left\langle\theta^{\prime}, \downarrow, K, \sigma^{\prime}, \theta_{K}, n+n^{\prime}, w^{\prime}\right\rangle$.

We also need to show that reductions leading to a failed observation are also preserved when appending a continuation.

Lemma 22. If $\left\langle\theta, C, K, \sigma, \theta_{K}, n, w\right\rangle \vdash^{*}\left\langle\theta^{\prime}, \downarrow,[], \downarrow, \theta_{K}^{\prime}, n+n^{\prime}, w^{\prime}\right\rangle$ then for all $K^{\prime \prime},\left\langle\theta, C, K @ K^{\prime \prime}, \sigma, \theta_{K}, n, w\right\rangle \vdash^{*}\left\langle\theta^{\prime}, C^{\prime},[], \downarrow, \theta_{K}^{\prime}, n+n^{\prime}, w^{\prime}\right\rangle$.

Proof. If $n^{\prime}=0$, the result follows trivially.

If $n^{\prime}>0$, then we have $\sigma \neq \downarrow$ (otherwise the initial configuration would not reduce), and so the last rule in the derivation of $\left\langle\theta, C, K, \sigma, \theta_{K}, n, w\right\rangle \vdash^{*}\left\langle\theta^{\prime}, C^{\prime}\right.$, $\left.K^{\prime}, \downarrow, \theta_{K}^{\prime}, n+n^{\prime}, w^{\prime}\right\rangle$ must have been (condition-false).

Hence, $\left\langle\theta, C, K, \sigma, \theta_{K}, n, w\right\rangle \vdash^{*}\left\langle\theta^{\prime}\right.$, observe $\left.(\phi), \hat{K}, \sigma^{\prime}, \theta_{K}^{\prime}, n+n^{\prime}-1, w^{\prime}\right\rangle \vdash$ $\left\langle\theta^{\prime}, \downarrow,[], \downarrow, \theta_{K}^{\prime}, n+n^{\prime}, w^{\prime}\right\rangle$, where $\sigma^{\prime} \neq \downarrow$ and $\sigma^{\prime}(\phi)=$ false. By Lemma [20, $\left\langle\theta, C, K @ K^{\prime \prime}, \sigma, \theta_{K}, n, w\right\rangle \vdash^{*}\left\langle\theta^{\prime}\right.$, observe $\left.(\phi), \hat{K} @ K^{\prime \prime}, \sigma^{\prime}, \theta_{K}^{\prime}, n+n^{\prime}-1, w^{\prime}\right\rangle$. By applying (condition-false) again, we get $\left\langle\theta^{\prime}\right.$, observe $(\phi), \hat{K} @ K^{\prime \prime}, \sigma^{\prime}, \theta_{K}^{\prime}, n+n^{\prime}-1$, $\left.w^{\prime}\right\rangle \vdash\left\langle\theta^{\prime}, \downarrow,[], \grave{\downarrow}, \theta_{K}^{\prime}, n+n^{\prime}, w^{\prime}\right\rangle$, as required.

Lemma 23. If $C_{1} \neq C_{1}^{\prime} ; C_{1}^{\prime \prime}$ and $\left\langle\pi_{L}(\theta), C_{1}\right.$, [], $\left.\sigma, \pi_{R}(\theta):: \theta_{K}, 0,1\right\rangle \vdash^{*}\left\langle\theta^{\prime}, \downarrow,[]\right.$, 々, $\left.\theta_{K}^{\prime}, n, w\right\rangle$, then $\left\langle\theta, C_{1} ; C_{2},[], \sigma, \theta_{K}, 0,1\right\rangle \vdash^{*}\left\langle\theta^{\prime}, \downarrow,[], \downarrow, \theta_{K}^{\prime}, n+1, w\right\rangle$.

Proof. By Lemma $22\left\langle\pi_{L}(\theta), C_{1},\left[C_{2}\right], \sigma, \pi_{R}(\theta):: \theta_{K}, 0,1\right\rangle \vdash^{*}\left\langle\theta^{\prime}, \downarrow,[], \downarrow, \theta_{K}^{\prime}, n\right.$, $w\rangle$. As $\left\langle\theta, C_{1} ; C_{2},[], \sigma, \theta_{K}, 0,1\right\rangle \vdash\left\langle\pi_{L}(\theta), C_{1},\left[C_{2}\right], \sigma, \pi_{R}(\theta):: \theta_{K}, 1,1\right\rangle$ by (seq), Lemma 17 yields $\left\langle\theta, C_{1} ; C_{2}\right.$, [], $\left.\sigma, \theta_{K}, 0,1\right\rangle \vdash^{*}\left\langle\theta^{\prime}, \downarrow\right.$, [], $\left.,, \theta_{K}^{\prime}, n+1, w\right\rangle$.

Sequencing We now use the above results to relate the final and intermediate configurations in the reduction of a statement $C_{1}$ to the intermediate configurations reached when reducing $C_{1} ; C_{2}$.

Lemma 24 (Context evaluation for simple sequencing). If $C_{1} \neq C_{1}^{\prime} ; C_{1}^{\prime \prime}$ and $\left\langle\theta, C_{1},[], \sigma, \theta_{K}, n, w\right\rangle \vdash_{\min }^{*}\left\langle\theta^{\prime}, \downarrow,[], \sigma^{\prime}, \theta_{K}, n+n^{\prime}, w^{\prime}\right\rangle$ and $\sigma^{\prime} \neq \downarrow$, then

$\left\langle\theta:: \pi_{L}\left(\theta_{K}\right), C_{1} ; C_{2},[], \sigma, \pi_{R}\left(\theta_{K}\right), n, w\right\rangle \vdash^{*}\left\langle\pi_{L}\left(\theta_{K}\right), C_{2},[], \sigma^{\prime}, \pi_{R}\left(\theta_{K}\right), n+n^{\prime}+2\right.$, $\left.w^{\prime}\right\rangle$.

Proof. By (seq): $\left\langle\theta:: \pi_{L}\left(\theta_{K}\right), C_{1} ; C_{2},[], \sigma, \pi_{R}\left(\theta_{K}\right), n, w\right\rangle \vdash\left\langle\theta, C_{1},\left[C_{2}\right], \sigma, \theta_{K}, n+1\right.$, $w\rangle$.

By Lemma 21 (and the fact that we can change $n$ ): $\left\langle\theta, C_{1},\left[C_{2}\right], \sigma, \theta_{K}, n+1\right.$, $w\rangle \vdash^{*}\left\langle\theta^{\prime}, \downarrow,\left[C_{2}\right], \sigma^{\prime}, \theta_{K},(n+1)+n^{\prime}, w^{\prime}\right\rangle$.

By (pop), $\left\langle\theta^{\prime}, \downarrow,\left[C_{2}\right], \sigma^{\prime}, \theta_{K},(n+1)+n^{\prime}, w^{\prime}\right\rangle \vdash\left\langle\pi_{L}\left(\theta_{K}\right), C_{2},[], \sigma^{\prime}, \pi_{R}\left(\theta_{K}\right)\right.$, $\left.(n+1)+n^{\prime}+1, w^{\prime}\right\rangle$, as required. 
Lemma 25. If $C_{1} \neq C_{1}^{\prime} ; C_{1}^{\prime \prime}$ and $\left\langle\theta, C_{1},[], \sigma, \theta_{K}, n, w\right\rangle \vdash^{*}\left\langle\theta^{\prime}, C^{\prime}, K, \sigma^{\prime}, \theta_{K}^{\prime}, n+\right.$ $\left.n^{\prime}, w^{\prime}\right\rangle$ and $\sigma^{\prime} \neq \downarrow$ and $\left(C^{\prime}, K^{\prime}\right) \neq(\downarrow,[])$, then $\left\langle\theta:: \pi_{L}\left(\theta_{K}\right), C_{1} ; C_{2},[], \sigma, \pi_{R}\left(\theta_{K}\right)\right.$, $n, w\rangle \vdash^{*}\left\langle\theta^{\prime}, C^{\prime}, K @\left[C_{2}\right], \sigma^{\prime}, \theta_{K}^{\prime}, n+n^{\prime}+1, w^{\prime}\right\rangle$.

Proof. By (seq), we have $\left\langle\theta:: \pi_{L}\left(\theta_{K}\right), C_{1} ; C_{2}\right.$, [], $\left.\sigma, \pi_{R}\left(\theta_{K}\right), n, w\right\rangle \vdash\left\langle\theta, C_{1},\left[C_{2}\right], \sigma\right.$, $\left.\theta_{K}, n+1, w\right\rangle$. Then, by Corollary团 $\left\langle\theta, C_{1},\left[C_{2}\right], \sigma, \theta_{K}, n+1, w\right\rangle \vdash^{*}\left\langle\theta^{\prime}, C^{\prime}, K @\left[C_{2}\right]\right.$, $\left.\sigma^{\prime}, \theta_{K}^{\prime}, n+n^{\prime}+1, w^{\prime}\right\rangle$, as required.

Splitting a sequence evaluation We now show that if a sequence $C_{1} ; C_{2}$ of statements evaluates under entropy $\theta$ to a proper state, then $C_{1}$ in itself must evaluate under $\pi_{L}(\theta)$, and that if the evaluation of $C_{1} ; C_{2}$ results in an error, then $C_{1}$ cannot diverge. These properties will be needed to show compositionality of the semantics.

To prove the first of the above properties, we first prove that if a configuration with an empty continuation reduces completely, then the continuation entropy $\theta_{K}$ in the final configuration will be identical to the original one (intermediate steps may extend $\theta_{K}$, but all sub-entropies added to $\theta_{K}$ will subsequently be removed). In the following lemma, we write $|K|$ for the length of list $K$.

Lemma 26. If $\left\langle\theta, C, K, \sigma, \hat{\theta_{K}}, n, w\right\rangle \vdash^{*}\left\langle\theta^{\prime}, \downarrow,[], \sigma^{\prime}, \theta_{K}^{\prime}, n+n^{\prime}, w^{\prime}\right\rangle$ and $\sigma^{\prime} \neq \xi$ and $\pi_{R}^{|K|}\left(\hat{\theta_{K}}\right)=\theta_{K}$, then $\theta_{K}^{\prime}=\theta_{K}$.

Proof. By induction on $n^{\prime}$ :

- Base case: $n^{\prime}=0$ : then obviously $|K|=0$ and $\hat{\theta_{K}}=\theta_{K}$, so the result follows trivially.

- Induction step: if $n^{\prime}>0$, then $\left\langle\theta, C, K, \sigma, \hat{\theta_{K}}, n, w\right\rangle \vdash\left\langle\theta^{\prime \prime}, C^{\prime \prime}, K^{\prime}, \sigma^{\prime \prime}, \theta_{K}^{\prime \prime}\right.$, $\left.n+1, w^{\prime \prime}\right\rangle \vdash^{*}\left\langle\theta^{\prime}, \downarrow,[], \sigma^{\prime}, \theta_{K}^{\prime}, n+n^{\prime}, w^{\prime}\right\rangle$.

Now we need to split on the first rule in this derivation chain.

If the first transition was derived with (seq), then $\left|K^{\prime}\right|=|K|+1$ and $\theta_{K}^{\prime \prime}=$ $\pi_{R}(\theta):: \hat{\theta_{K}}$. We have $\pi_{R}^{\left|K^{\prime}\right|}\left(\theta_{K}^{\prime \prime}\right)=\pi_{R}^{|K|+1}\left(\pi_{R}(\theta):: \hat{\theta_{K}}\right)=\pi_{R}^{|K|}\left(\pi_{R}\left(\pi_{R}(\theta)::\right.\right.$ $\left.\hat{\theta_{K}}\right)=\pi_{R}^{|K|}\left(\hat{\theta_{K}}\right)=\theta_{K}$, so by induction hypothesis, $\theta_{K}^{\prime}=\theta_{K}$.

If the first transition was derived with (pop), then $\left|K^{\prime}\right|=|K|-1$ and $\theta_{K}^{\prime \prime}=\pi_{R}\left(\hat{\theta_{K}}\right)$. Thus, $\pi_{R}^{\left|K^{\prime}\right|}\left(\theta_{K}^{\prime \prime}\right)=\pi_{R}^{\mid K \vdash 1}\left(\pi_{R}\left(\hat{\theta_{K}}\right)\right)=\pi_{R}^{|K|}\left(\hat{\theta_{K}}\right)=\hat{\theta_{K}}$, so by induction hypothesis, $\theta_{K}^{\prime}=\theta_{K}$.

Otherwise, we have $K^{\prime}=K$ (note that $\sigma^{\prime} \neq \downarrow$ implies $\sigma^{\prime \prime} \neq \downarrow$ ) and $\theta_{K}^{\prime \prime}=\hat{\theta_{K}}$, so $\pi_{R}^{\left|K^{\prime}\right|}\left(\theta_{K}^{\prime \prime}\right)=\theta_{K}$. By induction hypothesis, $\theta_{K}^{\prime}=\theta_{K}$.

Corollary 6. If $\left\langle\theta, C\right.$, [], $\left.\sigma, \theta_{K}, n, w\right\rangle \vdash^{*}\left\langle\theta^{\prime}, \downarrow,[], \sigma^{\prime}, \theta_{K}^{\prime}, n+n^{\prime}, w^{\prime}\right\rangle$ and $\sigma^{\prime} \neq \downarrow$, then $\theta_{K}^{\prime}=\theta_{K}$.

We now prove that if $C_{1} ; C_{2}$ successfully evaluates with entropy $\theta$, then $C_{1}$ also successfully evaluates with entropy $\pi_{L}(\theta)$.

Lemma 27 (Interpolation for Continuations). If $\left\langle\theta, C, K_{1} @ K_{2}, \sigma, \theta_{K}, n\right.$, $w\rangle \vdash^{*}\left\langle\theta^{\prime}, \downarrow,[], \sigma^{\prime}, \theta_{K}^{\prime}, n+n^{\prime}, w^{\prime}\right\rangle$ and $\sigma^{\prime} \neq \downarrow$, then $\left\langle\theta, C, K_{1}, \sigma, \theta_{K}, n, w\right\rangle \vdash^{*}\left\langle\theta^{\prime \prime}\right.$, $\left.\downarrow,[], \sigma^{\prime \prime}, \theta_{K}^{\prime \prime}, n+n^{\prime \prime}, w^{\prime \prime}\right\rangle$, where $\sigma^{\prime \prime} \neq \downarrow$. 
Proof. By induction on $n^{\prime}$.

- Base case: $n^{\prime}=0$ : in this case, $C=\downarrow$ and $K_{1}=K_{2}=$ [], so the result follows trivially.

- Induction step: suppose $\left\langle\theta, C, K_{1} @ K_{2}, \sigma, \theta_{K}, n, w\right\rangle \vdash\left\langle\hat{\theta}, \hat{C}, \hat{K}, \hat{\sigma}, \hat{\theta_{K}}, n+1\right.$, $\hat{w}\rangle \vdash^{*}\left\langle\theta^{\prime}, \downarrow,[], \sigma^{\prime}, \theta_{K}^{\prime}, n+n^{\prime}, w^{\prime}\right\rangle$.

If $\left\langle\theta, C, K_{1} @ K_{2}, \sigma, \theta_{K}, n, w\right\rangle \vdash\left\langle\hat{\theta}, \hat{C}, \hat{K}, \hat{\sigma}, \hat{\theta_{K}}, n+1, \hat{w}\right\rangle$ was derived with (seq), then $C=C_{1} ; C_{2}, C_{1} \neq C_{1}^{\prime} ; C_{1}^{\prime \prime}, \hat{K}=C_{2}:: K_{1} @ K_{2}, \hat{\theta}=\pi_{L}(\theta)$, $\hat{w}=w$ and $\hat{\theta_{K}}=\pi_{R}(\theta):: \theta_{K}$. By (seq), we have $\left\langle\theta, C_{1} ; C_{2}, K_{1}, \sigma, \theta_{K}, n\right.$, $w\rangle \vdash\left\langle\pi_{L}(\theta), C_{1}, C_{2}:: K_{1}, \sigma, \pi_{R}(\theta):: \theta_{K}, n+1, w\right\rangle$. By induction hypothesis, $\left\langle\pi_{L}(\theta), C_{1}, C_{2}:: K_{1}, \sigma, \pi_{R}(\theta):: \theta_{K}, n+1, w\right\rangle \vdash^{*}\left\langle\theta^{\prime \prime}, \downarrow,[], \sigma^{\prime \prime}, \theta_{K}^{\prime \prime}, n+n^{\prime \prime}, w^{\prime \prime}\right\rangle$ and $\sigma^{\prime \prime} \neq$ 々. Hence, $\left\langle\theta, C_{1} ; C_{2}, K_{1}, \sigma, \theta_{K}, n, w\right\rangle \vdash^{*}\left\langle\theta^{\prime \prime}, \downarrow,[], \sigma^{\prime \prime}, \theta_{K}^{\prime \prime}, n+n^{\prime \prime}\right.$, $\left.w^{\prime \prime}\right\rangle$, as required.

If $\left\langle\theta, C, K_{1} @ K_{2}, \sigma, \theta_{K}, n, w\right\rangle \vdash\left\langle\hat{\theta}, \hat{C}, \hat{K}, \hat{\sigma}, \hat{\theta_{K}}, n+1, \hat{w}\right\rangle$ was derived with (pop), then $C=\downarrow, K_{1} @ K_{2}=\hat{C}:: \hat{K}, \hat{w}=w, \hat{\theta}=\pi_{L}\left(\theta_{K}\right)$ and $\hat{\theta_{K}}=\pi_{R}\left(\theta_{K}\right)$.

- If $K_{1} \neq$ [], then $K_{1}=\hat{C}:: \hat{K}_{1}$ and $\hat{K}=\hat{K}_{1} @ K_{2}$ and we have $\langle\theta, \downarrow$, $\left.\hat{C}:: \hat{K}_{1}, \sigma, \theta_{K}, n, w\right\rangle \vdash\left\langle\pi_{L}\left(\theta_{K}\right), \hat{C}, \hat{K}_{1}, \sigma, \pi_{R}\left(\theta_{K}\right), n+1, w\right\rangle$. By induction hypothesis, $\left\langle\pi_{L}\left(\theta_{K}\right), \hat{C}, \hat{K}_{1}, \sigma, \pi_{R}\left(\theta_{K}\right), n+1, w\right\rangle \vdash^{*}\left\langle\theta^{\prime \prime}, \downarrow\right.$, [ [], $\sigma^{\prime \prime}, \theta_{K}^{\prime \prime}, n+$ $\left.n^{\prime \prime}, w^{\prime \prime}\right\rangle$ and $\sigma^{\prime \prime} \neq \downarrow$. Hence, we have $\left\langle\theta, \downarrow, \hat{C}:: \hat{K}_{1}, \sigma, \theta_{K}, n, w\right\rangle \vdash^{*}\left\langle\theta^{\prime \prime}, \downarrow\right.$, [], $\left.\sigma^{\prime \prime}, \theta_{K}^{\prime \prime}, n+n^{\prime \prime}, w^{\prime \prime}\right\rangle$.

- If $K_{1}=$ [], then trivially $\left\langle\theta, \downarrow\right.$, [], $\left.\sigma, \theta_{K}, n, w\right\rangle \vdash^{*}\left\langle\theta, \downarrow\right.$, [], $\left.\sigma, \theta_{K}, n, w\right\rangle$ in zero steps.

Otherwise, $\hat{K}=K_{1} @ K_{2}$ and $\hat{\theta_{K}}=\theta_{K}$ and by inspection of the reduction rules, $\left\langle\theta, C, K_{1}, \sigma, \theta_{K}, n, w\right\rangle \vdash\left\langle\hat{\theta}, \hat{C}, K_{1}, \hat{\sigma}, \theta_{K}, n+1, \hat{w}\right\rangle$. Hence, by induction hypothesis, $\left\langle\theta, C, K_{1}, \sigma, \theta_{K}, n, w\right\rangle \vdash\left\langle\hat{\theta}, \hat{C}, K_{1}, \hat{\sigma}, \theta_{K}, n+1, \hat{w}\right\rangle \vdash^{*}\left\langle\theta^{\prime \prime}, \downarrow,[], \sigma^{\prime \prime}\right.$, $\left.\theta_{K}^{\prime \prime}, n+n^{\prime \prime}, w^{\prime \prime}\right\rangle$ and $\sigma^{\prime \prime} \neq$, as required.

Lemma 28 (Interpolation). If $C_{1} \neq C_{1}^{\prime} ; C_{1}^{\prime \prime}$ and $\left\langle\theta, C_{1} ; C_{2}\right.$, [], $\left.\sigma, \theta_{K}, n, w\right\rangle \vdash^{*}$ $\left\langle\theta^{\prime}, \downarrow,[], \sigma^{\prime}, \theta_{K}, n+n^{\prime}, w^{\prime}\right\rangle$ and $\sigma^{\prime} \neq \downarrow$, then $\left\langle\pi_{L}(\theta), C_{1},[], \sigma, \pi_{R}(\theta):: \theta_{K}, n, w\right\rangle \vdash^{*}$ $\left\langle\theta^{\prime \prime}, \downarrow,[], \sigma^{\prime \prime}, \theta_{K}, n+n^{\prime \prime}, w^{\prime \prime}\right\rangle$, where $\sigma^{\prime \prime} \neq \downarrow$.

Proof. The first rule applied in the derivation of $\left\langle\theta, C_{1} ; C_{2}\right.$, [], $\left.\sigma, \theta_{K}, n, w\right\rangle \vdash^{*}\left\langle\theta^{\prime}\right.$, $\downarrow$, [], $\left.\sigma^{\prime}, \theta_{K}, n+n^{\prime}, w^{\prime}\right\rangle$ is (seq), which gives $\left\langle\theta, C_{1} ; C_{2}\right.$, [], $\left.\sigma, \theta_{K}, n, w\right\rangle \vdash\left\langle\pi_{L}(\theta), C_{1}\right.$, $\left.\left[C_{2}\right], \sigma, \pi_{R}(\theta):: \theta_{K}, n+1, w\right\rangle$. Hence, $\left\langle\pi_{L}(\theta), C_{1},\left[C_{2}\right], \sigma, \pi_{R}(\theta):: \theta_{K}, n+1, w\right\rangle \vdash^{*}$ $\left\langle\theta^{\prime}, \downarrow,[], \sigma^{\prime}, \theta_{K}, n+n^{\prime}, w^{\prime}\right\rangle$. By applying Lemma 27]with $K_{1}=[]$ and Corollary 6 , we get $\left\langle\pi_{L}(\theta), C_{1},[], \sigma, \pi_{R}(\theta):: \theta_{K}, n+1, w\right\rangle \vdash^{*}\left\langle\theta^{\prime \prime}, \downarrow,[], \sigma^{\prime \prime}, \pi_{R}(\theta):: \theta_{K}, n+n^{\prime \prime}\right.$, $\left.w^{\prime \prime}\right\rangle$, where $\sigma^{\prime \prime} \neq \downarrow$, as required.

Finally, we show that if the evaluation of $C_{1} ; C_{2}$ with entropy $\theta$ yields an error, then the evaluation of $C_{1}$ under $\pi_{L}(\theta)$ either terminates successfully or also results in an error (depending on where the error in the evaluation of $C_{1} ; C_{2}$ occurred) - at any rate, $C_{1}$ does not diverge.

Lemma 29. If $C_{1} \neq C_{1}^{\prime} ; C_{2}^{\prime}$ and $\left\langle\theta, C_{1} ; C_{2}\right.$, [], $\left.\sigma, \theta_{K}, 0,1\right\rangle \vdash^{*}\left\langle\theta^{\prime}, C^{\prime}, K, \sigma^{\prime}, \theta_{K}^{\prime}, n\right.$, $w\rangle \nvdash$, then either $\left\langle\pi_{L}(\theta), C_{1},[], \sigma, \pi_{R}(\theta):: \theta_{K}, 0,1\right\rangle \vdash^{*}\left\langle\theta^{\prime \prime}, \downarrow,[], \sigma^{\prime \prime}, \theta_{K}, n^{\prime}, w^{\prime}\right\rangle$ or $\left\langle\pi_{L}(\theta), C_{1},[], \sigma, \pi_{R}(\theta):: \theta_{K}, 0,1\right\rangle \vdash^{*}\left\langle\theta^{\prime \prime}, C_{1}^{\prime \prime}, K^{\prime \prime}, \sigma^{\prime \prime}, \theta_{K}, n^{\prime}, w^{\prime}\right\rangle \nvdash$. 
Proof. The statement in the lemma is equivalent to saying that it is not the case that for all $k,\left\langle\pi_{L}(\theta), C_{1}\right.$, [], $\left.\sigma, \pi_{R}(\theta):: \theta_{K}, 0,1\right\rangle \vdash^{*}\left\langle\theta^{\prime \prime}, C_{1}^{\prime \prime}, K^{\prime \prime}, \sigma^{\prime \prime}, \pi_{R}(\theta):: \theta_{K}\right.$, $\left.k, w^{\prime}\right\rangle$ with $\left(C_{1}^{\prime \prime}, K^{\prime \prime}\right) \neq(\downarrow,[])$. Suppose for contradiction that the negation of this statement holds. By (seq), we have $\left\langle\theta, C_{1} ; C_{2}\right.$, [], $\left.\sigma, \theta_{K}, 0,1\right\rangle \vdash\left\langle\pi_{L}(\theta), C_{1},\left[C_{2}\right], \sigma\right.$, $\left.\pi_{R}(\theta):: \theta_{K}, 1,1\right\rangle$, so $\left\langle\pi_{L}(\theta), C_{1},\left[C_{2}\right], \sigma, \pi_{R}(\theta):: \theta_{K}, 1,1\right\rangle \vdash^{*}\left\langle\theta^{\prime}, C^{\prime}, K, \sigma^{\prime}, \theta_{K}^{\prime}, n\right.$, $w\rangle$.

Take $k=n-1$. Then we have $\left\langle\pi_{L}(\theta), C_{1},[], \sigma, \pi_{R}(\theta):: \theta_{K}, 0,1\right\rangle \vdash^{*}\left\langle\theta^{\prime \prime}\right.$, $\left.C_{1}^{\prime \prime}, K^{\prime \prime}, \sigma^{\prime \prime}, \pi_{R}(\theta):: \theta_{K}, n-1, w^{\prime}\right\rangle \vdash\left\langle\hat{\theta}, \hat{C_{1}}, \hat{K}, \hat{\sigma}, \hat{\theta_{K}}, n, \hat{w}\right\rangle$, where $\sigma^{\prime \prime} \neq$ 々 (otherwise the middle configuration would not reduce) and $\left(C_{1}^{\prime \prime}, K^{\prime \prime}\right) \neq(\downarrow,[])$. By Corollary 4, we have $\left\langle\pi_{L}(\theta), C_{1},\left[C_{2}\right], \sigma, \pi_{R}(\theta):: \theta_{K}, 1,1\right\rangle \vdash^{*}\left\langle\theta^{\prime \prime}, C_{1}^{\prime \prime}, K^{\prime \prime} @\left[C_{2}\right]\right.$, $\left.\sigma^{\prime \prime}, \pi_{R}(\theta):: \theta_{K}, n, w^{\prime}\right\rangle$. Hence, $\left\langle\theta, C_{1} ; C_{2},[], \sigma, \theta_{K}, 0,1\right\rangle \vdash^{*}\left\langle\theta^{\prime \prime}, C_{1}^{\prime \prime}, K^{\prime \prime} @\left[C_{2}\right], \sigma^{\prime \prime}\right.$, $\left.\pi_{R}(\theta):: \theta_{K}, n, w^{\prime}\right\rangle$ and $\left\langle\theta^{\prime \prime}, C_{1}^{\prime \prime}, K^{\prime \prime} @\left[C_{2}\right], \sigma^{\prime \prime}, \pi_{R}(\theta):: \theta_{K}, n, w^{\prime}\right\rangle=\left\langle\theta^{\prime}, C^{\prime}, K, \sigma^{\prime}\right.$, $\left.\theta_{K}^{\prime}, n, w\right\rangle$, since reduction is deterministic. By Lemma 18 this implies that $\left\langle\theta^{\prime}\right.$, $\left.C^{\prime}, K, \sigma^{\prime}, \theta_{K}^{\prime}, n, w\right\rangle$ reduces, contradicting the assumption.

Corollary 7. If $C_{1} \neq C_{1}^{\prime} ; C_{2}^{\prime}$ and $\left\langle\theta, C_{1} ; C_{2}\right.$, [], $\left.\sigma, \theta_{K}, 0,1\right\rangle \vdash^{*}\left\langle\theta^{\prime}, C^{\prime}, K, \sigma^{\prime}, \theta_{K}^{\prime}\right.$, $n, w\rangle \nvdash$, then $\mathbf{O}_{C_{1}}^{\sigma}\left(\pi_{L}(\theta)\right) \neq \uparrow$.

\section{D.2 Properties of the semantic functions}

Compositionality of sequencing. A desirable and useful property of the semantic functions is compositionality with respect to sequencing, i.e., the ability to define $\mathbf{O}_{C_{1} ; C_{2}}^{\sigma}$ in terms of $\mathbf{O}_{C_{1}}^{\sigma_{1}}$ and $\mathbf{O}_{C_{2}}^{\sigma_{2}}$ for some states $\sigma_{1}$ and $\sigma_{2}$. Similarly for $\mathbf{S C}_{C_{1} ; C_{2}}^{\sigma}$. We can easily express the semantics of $C_{1} ; C_{2}$ in terms of the semantics of $C_{1}$ and $C_{2}$ if $C_{1}$ is not a sequence of statements. (Recall the explanation of the rule (seq).)

Proposition 1 (Simple sequencing for final states). If $C_{1} \neq C_{1}^{\prime} ; C_{2}^{\prime}$, then:

$$
\mathbf{O}_{C_{1} ; C_{2}}^{\sigma}(\theta)=\mathbf{O}_{C_{2}}^{\tau}\left(\pi_{R}(\theta)\right) \quad \text { and } \quad \mathbf{S C}_{C_{1} ; C_{2}}^{\sigma}(\theta)=\mathbf{S C}_{C_{1}}^{\sigma}\left(\pi_{L}(\theta)\right) \cdot \mathbf{S C}_{C_{2}}^{\tau}\left(\pi_{R}(\theta)\right)
$$

where $\tau$ stands for the state $\mathbf{O}_{C_{1}}^{\sigma}\left(\pi_{L}(\theta)\right)$.

Below, we prove Proposition 1, To simplify presentation, we split it into two separate lemmas, one concerning final states and one concerning scores.

Lemma 30 (Simple sequencing for final states). If $C_{1} \neq C_{1}^{\prime} ; C_{2}^{\prime}$, then $\mathbf{O}_{C_{1} ; C_{2}}^{\sigma}(\theta)=\mathbf{O}_{C_{2}}^{\mathbf{O}_{C_{1}}^{\sigma}\left(\pi_{L}(\theta)\right)}\left(\pi_{R}(\theta)\right)$

Proof. If $\sigma=\uparrow$, then $L H S=R H S=\uparrow$ directly by definition.

If $\sigma=\xi$, the result also follows trivially, so let us suppose $\sigma \neq \downarrow$ and $\sigma \neq \uparrow$.

We need to consider several cases:

- If $\mathbf{O}_{C_{1}}^{\sigma}\left(\pi_{L}(\theta)\right)=$ 々, then $\left\langle\pi_{L}(\theta), C_{1}\right.$, [], $\left.\sigma, \pi_{R}(\theta):: \theta_{K}, 0,1\right\rangle \vdash^{*}\left\langle\theta^{\prime}, C_{1}^{\prime}, K, \tau\right.$, $\left.\theta_{K}^{\prime}, n, w\right\rangle \nvdash$. By (seq), we have $\left\langle\theta, C_{1} ; C_{2}\right.$, [], $\left.\sigma, \theta_{K}, 0,1\right\rangle \vdash\left\langle\pi_{L}(\theta), C_{1},\left[C_{2}\right], \sigma\right.$, $\left.\pi_{R}(\theta):: \theta_{K}, 1,1\right\rangle$.

If $\tau \neq \downarrow$, then by Lemmas 20 and [17, $\left\langle\pi_{L}(\theta), C_{1},\left[C_{2}\right], \sigma, \pi_{R}(\theta):: \theta_{K}, 1\right.$, $1\rangle \vdash^{*}\left\langle\theta^{\prime}, C_{1}^{\prime}, K @\left[C_{2}\right], \tau, \theta_{K}^{\prime}, n+1, w\right\rangle \nvdash$. Moreover, $\left\langle\theta^{\prime}, C_{1}^{\prime}, K, \tau, \theta_{K}^{\prime}, n, w\right\rangle \nvdash$ 
implies $C_{1}^{\prime} \neq \downarrow$ (because otherwise the configuration would reduce by (final) or (pop)), so by inspection, $\left\langle\theta^{\prime}, C_{1}^{\prime}, K @\left[C_{2}\right], \tau, \theta_{K}^{\prime}, n+1, w\right\rangle \nvdash$. Thus, $\mathbf{O}_{C_{1} ; C_{2}}^{\sigma}(\theta)=$ 々.

If $\tau=\downarrow$, then $C_{1}^{\prime}=\downarrow, K=[]$ and by Lemmas 22 and 17 we have $\left\langle\pi_{L}(\theta), C_{1}\right.$, $\left.\left[C_{2}\right], \sigma, \pi_{R}(\theta):: \theta_{K}, 1,1\right\rangle \vdash^{*}\left\langle\theta^{\prime}, \downarrow,[], \downarrow, \theta_{K}^{\prime}, n+1, w\right\rangle \nvdash$. Hence, $\mathbf{O}_{C_{1} ; C_{2}}^{\sigma}(\theta)=$ 々.

- If $\mathbf{O}_{C_{1}}^{\sigma}\left(\pi_{L}(\theta)\right)=\uparrow$, then $R H S=\uparrow$. Moreover, we have neither $\left\langle\pi_{L}(\theta), C_{1}\right.$, [], $\left.\sigma, \pi_{R}(\theta):: \theta_{K}, 0,1\right\rangle \vdash^{*}\left\langle\theta^{\prime}, \downarrow,[], \tau, \theta_{K}, n, w\right\rangle$ nor $\left\langle\pi_{L}(\theta), C_{1},[], \sigma, \pi_{R}(\theta):: \theta_{K}\right.$, $0,1\rangle \vdash^{*}\left\langle\theta^{\prime}, C^{\prime}, K, \tau, \theta_{K}^{\prime}, n, w\right\rangle \nvdash$.

Now, suppose for contradiction that LHS $\neq \uparrow$. Then we have either $\langle\theta$, $\left.C_{1} ; C_{2},[], \sigma, \theta_{K}, 0,1\right\rangle \vdash^{*}\left\langle\theta^{\prime}, \downarrow,[], \tau, \theta_{K}, n, w\right\rangle\left(\right.$ with $\tau \neq$ 々) or $\left\langle\theta, C_{1} ; C_{2}\right.$, [], $\left.\sigma, \theta_{K}, 0,1\right\rangle \vdash^{*}\left\langle\theta^{\prime}, C^{\prime}, K, \tau, \theta_{K}^{\prime}, n, w\right\rangle \nvdash$.

First, suppose that $\left\langle\theta, C_{1} ; C_{2},[], \sigma, \theta_{K}, 0,1\right\rangle \vdash^{*}\left\langle\theta^{\prime}, \downarrow,[], \tau, \theta_{K}, n, w\right\rangle$, where $\tau \neq \downarrow$. By Lemma 28 this implies that $\left\langle\pi_{L}(\theta), C_{1},[], \sigma, \pi_{R}(\theta):: \theta_{K}, 0,1\right\rangle \vdash^{*}$ $\left\langle\theta^{\prime \prime}, \downarrow,[], \tau^{\prime}, \pi_{R}(\theta):: \theta_{K}, n^{\prime}, w^{\prime}\right\rangle$ and so $\mathbf{O}_{C_{1}}^{\sigma}\left(\pi_{L}(\theta)\right)=\tau^{\prime} \neq \uparrow$, contradicting the assumption.

If $\left\langle\theta, C_{1} ; C_{2}\right.$, [], $\left.\sigma, \theta_{K}, 0,1\right\rangle \vdash^{*}\left\langle\theta^{\prime}, C^{\prime}, K, \tau, \theta_{K}^{\prime}, n, w\right\rangle \nvdash$, then by Corollary $\mathbf{7}$. we get a contradiction.

- If $\mathbf{O}_{C_{1}}^{\sigma}\left(\pi_{L}(\theta)\right) \notin\{\xi, \uparrow\}$, but $\mathbf{O}_{C_{2}}^{\mathbf{O}_{C_{1}}^{\sigma}\left(\pi_{L}(\theta)\right)}\left(\pi_{R}(\theta)\right)=$, we have $\left\langle\pi_{L}(\theta), C_{1}\right.$, [], $\left.\sigma, \pi_{R}(\theta):: \theta_{K}, 0,1\right\rangle \vdash_{\min }^{*}\left\langle\theta^{\prime}, \downarrow,[], \tau^{\prime}, \pi_{R}(\theta):: \theta_{K}, n, w\right\rangle$ for some $\tau^{\prime} \neq \downarrow$, where $\mathbf{O}_{C_{1}}^{\sigma}\left(\pi_{L}(\theta)\right)=\tau^{\prime}$, and $\left\langle\pi_{R}(\theta), C_{2},[], \tau^{\prime}, \theta_{K}, 0,1\right\rangle \vdash^{*}\left\langle\theta^{\prime \prime}, C^{\prime \prime}, K^{\prime}, \tau, \theta_{K}^{\prime}\right.$, $\left.n^{\prime}, w^{\prime}\right\rangle \nvdash$. By Lemma 24] $\left\langle\theta, C_{1} ; C_{2},[], \sigma, \theta_{K}, 0,1\right\rangle \vdash^{*}\left\langle\pi_{R}(\theta), C_{2},[], \tau^{\prime}, \theta_{K}, n+\right.$ $2, w\rangle$. By Lemma 17] $\left\langle\pi_{R}(\theta), C_{2},[], \tau^{\prime}, \theta_{K}, n+2, w\right\rangle \vdash^{*}\left\langle\theta^{\prime \prime}, C^{\prime \prime}, K^{\prime}, \tau, \theta_{K}^{\prime}, n+\right.$ $\left.2+n^{\prime}, w w^{\prime}\right\rangle$, where the last configuration clearly does not reduce, as changing

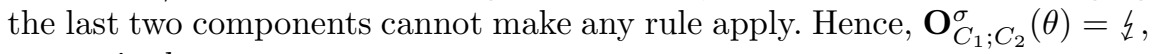
as required.

- If $\mathbf{O}_{C_{1}}^{\sigma}\left(\pi_{L}(\theta)\right) \notin\{\not, \uparrow\}$, but $\mathbf{O}_{C_{2}}^{\mathbf{O}_{C_{1}}^{\sigma}\left(\pi_{L}(\theta)\right)}\left(\pi_{R}(\theta)\right)=\uparrow$, we have again $\left\langle\pi_{L}(\theta)\right.$, $\left.C_{1},[], \sigma, \pi_{R}(\theta):: \theta_{K}, 0,1\right\rangle \vdash_{\min }^{*}\left\langle\theta^{\prime}, \downarrow,[], \tau^{\prime}, \pi_{R}(\theta):: \theta_{K}, n, w\right\rangle$ for some $\tau^{\prime} \neq \downarrow$. Again, by Lemma 24. we have $\left\langle\theta, C_{1} ; C_{2}\right.$, [], $\left.\sigma, \theta_{K}, 0,1\right\rangle \vdash^{*}\left\langle\pi_{R}(\theta), C_{2}\right.$, [], $\tau^{\prime}$, $\left.\theta_{K}, n+2, w\right\rangle$, but we have neither $\left\langle\pi_{R}(\theta), C_{2},[], \tau^{\prime}, \theta_{K}, 0,1\right\rangle \vdash^{*}\left\langle\theta^{\prime \prime}, \downarrow,[], \tau^{\prime \prime}\right.$, $\left.\theta_{K}, n^{\prime}, w^{\prime}\right\rangle$ nor $\left\langle\pi_{R}(\theta), C_{2},[], \tau^{\prime}, \theta_{K}, 0,1\right\rangle \vdash^{*}\left\langle\theta^{\prime \prime}, C^{\prime \prime}, K^{\prime}, \tau, \theta_{K}^{\prime}, n^{\prime}, w^{\prime}\right\rangle \nvdash$.

Suppose for contradiction that LHS $\neq \uparrow$. Then we have either $\left\langle\theta, C_{1} ; C_{2}\right.$, [], $\left.\sigma, \theta_{K}, 0,1\right\rangle \vdash^{*}\left\langle\theta^{\prime}, \downarrow\right.$, [] $\left.\tau, \theta_{K}, \hat{n}, \hat{w}\right\rangle($ with $\tau \neq \downarrow)$ or $\left\langle\theta, C_{1} ; C_{2}\right.$, [] $, \sigma, \theta_{K}, 0$, $1\rangle \vdash^{*}\left\langle\theta^{\prime}, C^{\prime}, K, \tau, \theta_{K}^{\prime}, \hat{n}, \hat{w}\right\rangle \nvdash$.

In the former case, the determinicity of reduction implies $\left\langle\pi_{R}(\theta), C_{2}\right.$, [], $\tau^{\prime}$, $\left.\theta_{K}, n+2, w\right\rangle \vdash^{*}\left\langle\theta^{\prime}, \downarrow,[], \tau, \theta_{K}, \hat{n}, \hat{w}\right\rangle$, so by Lemma [17, $\left\langle\pi_{R}(\theta), C_{2},[], \tau^{\prime}, \theta_{K}\right.$, $0,1\rangle \vdash^{*}\left\langle\theta^{\prime}, \downarrow,[], \tau, \theta_{K}, \hat{n}-n-2, \hat{w} / w\right\rangle$, which contradicts the assumption.

Similarly, in the latter case, $\left\langle\pi_{R}(\theta), C_{2}\right.$, [], $\left.\tau^{\prime}, \theta_{K}, n+2, w\right\rangle \vdash^{*}\left\langle\theta^{\prime}, C^{\prime}, K, \tau\right.$, $\left.\theta_{K}^{\prime}, \hat{n}, \hat{w}\right\rangle \nvdash$, which violates the assumption.

Hence, $\mathbf{O}_{C_{1} ; C_{2}}^{\sigma}(\theta)=\uparrow$.

- Finally, suppose that $\mathbf{O}_{C_{1}}^{\sigma}\left(\pi_{L}(\theta)\right) \notin\{\not, \uparrow\}$ and $\mathbf{O}_{C_{2}}^{\mathbf{O}_{C_{1}}^{\sigma}\left(\pi_{L}(\theta)\right)}\left(\pi_{R}(\theta)\right) \notin$ $\{\not, \uparrow\}$. Then we have again $\left\langle\pi_{L}(\theta), C_{1},[], \sigma, \pi_{R}(\theta):: \theta_{K}, 0,1\right\rangle \vdash_{\min }^{*}\left\langle\theta^{\prime}\right.$, $\left.\downarrow,[], \tau^{\prime}, \pi_{R}(\theta):: \theta_{K}, n^{\prime}, w^{\prime}\right\rangle$ for some $\tau^{\prime} \neq \downarrow$ and $\left\langle\theta, C_{1} ; C_{2},[], \sigma, \theta_{K}, 0\right.$, $1\rangle \vdash^{*}\left\langle\pi_{R}(\theta), C_{2},[], \tau^{\prime}, \theta_{K}, n^{\prime}, w^{\prime}\right\rangle$ by Lemma 24]. Since $\mathbf{O}_{C_{1}}^{\sigma}\left(\pi_{L}(\theta)\right)=\tau^{\prime}$ and $\mathbf{O}_{C_{2}}^{\mathbf{O}_{C_{1}}^{\sigma}\left(\pi_{L}(\theta)\right)}\left(\pi_{R}(\theta)\right)=\tau^{\prime \prime} \neq$ 々, we have $\left\langle\pi_{R}(\theta), C_{2},[], \tau^{\prime}, \theta_{K}, n^{\prime}, w^{\prime}\right\rangle \vdash^{*}\left\langle\theta^{\prime \prime}, \downarrow\right.$, [], $\left.\tau^{\prime \prime}, \theta_{K}, n^{\prime \prime}, w^{\prime \prime}\right\rangle$. This also implies that 


$$
\begin{aligned}
& \left\langle\theta, C_{1} ; C_{2},[], \sigma, \theta_{K}, 0,1\right\rangle \vdash^{*}\left\langle\theta^{\prime \prime}, \downarrow,[], \tau^{\prime \prime}, \theta_{K}, n^{\prime \prime}, w^{\prime \prime}\right\rangle, \text { and so } \mathbf{O}_{C_{1} ; C_{2}}^{\sigma}(\theta)= \\
& \tau^{\prime \prime}=\mathbf{O}_{C_{2}}^{\sigma}\left(\pi_{L}(\theta)\right)\left(\pi_{R}(\theta)\right) .
\end{aligned}
$$

Lemma 31 (Simple sequencing for scores). If $C_{1} \neq C_{1}^{\prime} ; C_{2}^{\prime}$ then $\mathbf{S C}_{C_{1} ; C_{2}}^{\sigma}(\theta)=$ $\mathbf{S C}_{C_{1}}^{\sigma}\left(\pi_{L}(\theta)\right) \cdot \mathbf{S C}_{C_{2}}^{\mathbf{O}_{C_{1}}^{\sigma}\left(\pi_{L}(\theta)\right)}\left(\pi_{R}(\theta)\right)$

Proof. If $\sigma=\xi$ or $\sigma=\uparrow$, the property holds trivially, so let us assume $\sigma \notin\{\not, \uparrow\}$. We need to consider three cases:

- If $\mathbf{O}_{C_{1}}^{\sigma}\left(\pi_{L}(\theta)\right)=\sigma^{\prime} \notin\{\xi, \uparrow\}$, then $\left\langle\pi_{L}(\theta), C_{1},[], \sigma, \pi_{R}(\theta):: \theta_{K}, 0,1\right\rangle \vdash_{\min }^{*}\left\langle\theta^{\prime}\right.$, $\left.\downarrow,[], \sigma^{\prime}, \pi_{R}(\theta):: \theta_{K}, n, w\right\rangle$ and $\mathbf{S C}_{C_{1}}^{\sigma}\left(\pi_{L}(\theta)\right)=w$.

By Lemma 24, $\left\langle\theta, C_{1} ; C_{2},[], \sigma, \theta_{K}, 0,1\right\rangle \vdash^{*}\left\langle\pi_{R}(\theta), C_{2},[], \sigma^{\prime}, \theta_{K}, n+2, w\right\rangle$.

Now, fix a $k \geq 0$.

- If $\left\langle\pi_{R}(\theta), C_{2},[], \sigma^{\prime}, \theta_{K}, 0,1\right\rangle \quad \vdash^{*} \quad\left\langle\theta^{\prime \prime}, C_{2}^{\prime}, K, \sigma^{\prime \prime}, \theta_{K}^{\prime}, k, w^{\prime}\right\rangle$, then $\mathbf{S C}_{C_{2}}^{\mathbf{O}_{C_{1}}^{\sigma}\left(\pi_{L}(\theta)\right)}\left(\pi_{R}(\theta), k\right)=w^{\prime}$. By Lemma 17 $\left\langle\pi_{R}(\theta), C_{2},[], \sigma^{\prime}, \theta_{K}\right.$, $n+2, w\rangle \vdash^{*}\left\langle\theta^{\prime \prime}, C_{2}^{\prime}, K, \sigma^{\prime \prime}, \theta_{K}^{\prime}, n+2+k, w w^{\prime}\right\rangle$, which implies $\langle\theta$, $\left.C_{1} ; C_{2},[], \sigma, \theta_{K}, 0,1\right\rangle \vdash^{*}\left\langle\theta^{\prime \prime}, C_{2}^{\prime}, K, \sigma^{\prime \prime}, \theta_{K}^{\prime}, n+2+k, w w^{\prime}\right\rangle$, and so $\mathbf{S C}_{C_{1} ; C_{2}}^{\sigma}(\theta, n+2+k)=w w^{\prime}=\mathbf{S C}_{C_{1}}^{\sigma}\left(\pi_{L}(\theta)\right) \mathbf{S C}_{C_{2}}^{\mathbf{O}_{C_{1}}^{\sigma}\left(\pi_{L}(\theta)\right)}\left(\pi_{R}(\theta), k\right)$.

- If there is no configuration $\left\langle\theta^{\prime \prime}, C_{2}^{\prime}, K, \sigma^{\prime \prime}, \theta_{K}^{\prime}, k, w^{\prime}\right\rangle$ such that $\left\langle\pi_{R}(\theta), C_{2},[], \sigma^{\prime}, \theta_{K}, 0,1\right\rangle \quad \vdash^{*} \quad\left\langle\theta^{\prime \prime}, C_{2}^{\prime}, K, \sigma^{\prime \prime}, \theta_{K}^{\prime}, k, w^{\prime}\right\rangle$, then $\mathbf{S C}_{C_{2}}^{\mathbf{O}_{C_{1}}^{\sigma}\left(\pi_{L}(\theta)\right)}\left(\pi_{R}(\theta), k\right)=0$. If we had $\left\langle\theta, C_{1} ; C_{2}\right.$, [], $\left.\sigma, \theta_{K}, 0,1\right\rangle \vdash^{*}\left\langle\theta^{\prime \prime}\right.$, $\left.C_{2}^{\prime}, K, \sigma^{\prime \prime}, \theta_{K}^{\prime}, n+2+k, w w^{\prime}\right\rangle$, then, by determinacy of reduction, $\left\langle\pi_{R}(\theta), C_{2},[], \sigma^{\prime}, \theta_{K}, n+2, w\right\rangle \vdash^{*}\left\langle\theta^{\prime \prime}, C_{2}^{\prime}, K, \sigma^{\prime \prime}, \theta_{K}^{\prime}, n+2+k, w w^{\prime}\right\rangle$. Вy Lemma 17 and Lemma 16 (which ensures $w>0$ ), $\left\langle\pi_{R}(\theta), C_{2}\right.$, [], $\sigma^{\prime}, \theta_{K}$, $0,1\rangle \vdash^{*}\left\langle\theta^{\prime \prime}, C_{2}^{\prime}, K, \sigma^{\prime \prime}, \theta_{K}^{\prime}, k, w^{\prime}\right\rangle$, which contradicts the assumption. Hence, there is no configuration $\left\langle\theta^{\prime \prime}, C_{2}^{\prime}, K, \sigma^{\prime \prime}, \theta_{K}^{\prime}, n+2+k, w w^{\prime}\right\rangle$ such that $\left\langle\theta, C_{1} ; C_{2},[], \sigma, \theta_{K}, 0,1\right\rangle \vdash^{*}\left\langle\theta^{\prime \prime}, C_{2}^{\prime}, K, \sigma^{\prime \prime}, \theta_{K}^{\prime}, n+2+k, w w^{\prime}\right\rangle$, and so $\mathbf{S C}_{C_{1} ; C_{2}}^{\sigma}(\theta, n+2+k)=0$.

In either case, $\mathbf{S C}_{C_{1} ; C_{2}}^{\sigma}(\theta, n+2+k)=\mathbf{S C}_{C_{1}}^{\sigma}\left(\pi_{L}(\theta)\right) \cdot \mathbf{S C}_{C_{2}}^{\mathbf{O}_{C_{1}}^{\sigma}\left(\pi_{L}(\theta)\right)}\left(\pi_{R}(\theta), k\right)$ for all $k \geq 0$. Thus, we have

$$
\begin{aligned}
\mathbf{S C}_{C_{1} ; C_{2}}^{\sigma}(\theta) & =\lim _{n \rightarrow \infty} \mathbf{S C}_{C_{1} ; C_{2}}^{\sigma}(\theta, n) \\
& =\lim _{k \rightarrow \infty} \mathbf{S C}_{C_{1} ; C_{2}}^{\sigma}(\theta, n+2+k) \\
& =\lim _{k \rightarrow \infty} \mathbf{S C}_{C_{1}}^{\sigma}\left(\pi_{L}(\theta)\right) \cdot \mathbf{S C}_{C_{2}}^{\mathbf{O}_{C_{1}}^{\sigma}\left(\pi_{L}(\theta)\right)}\left(\pi_{R}(\theta), k\right) \\
& =\mathbf{S C}_{C_{1}}^{\sigma}\left(\pi_{L}(\theta)\right) \lim _{k \rightarrow \infty} \mathbf{S C}_{C_{2}}^{\mathbf{O}_{C_{1}}^{\sigma}\left(\pi_{L}(\theta)\right)}\left(\pi_{R}(\theta), k\right) \\
& =\mathbf{S C}_{C_{1}}^{\sigma}\left(\pi_{L}(\theta)\right) \mathbf{S C}_{C_{2}}^{\mathbf{O}_{C_{1}}^{\sigma}\left(\pi_{L}(\theta)\right)}\left(\pi_{R}(\theta)\right)
\end{aligned}
$$

- If $\mathbf{O}_{C_{1}}^{\sigma}\left(\pi_{L}(\theta)\right)=$ ұ, then $\mathbf{S C}_{C_{2}}^{\mathbf{O}_{C_{1}}^{\sigma}\left(\pi_{L}(\theta)\right)}\left(\pi_{R}(\theta)\right)=0$, so $R H S=0$. Moreover, we have $\left\langle\pi_{L}(\theta), C_{1}\right.$, [], $\left.\sigma, \pi_{R}(\theta):: \theta_{K}, 0,1\right\rangle \vdash^{*}\left\langle\theta^{\prime}, C^{\prime}, K, \tau, \theta_{K}^{\prime}, n, w\right\rangle \nvdash$.

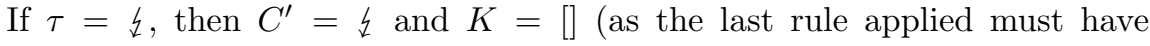


been (condition-false)), so by Lemma 23. $\left\langle\theta, C_{1} ; C_{2},[], \sigma, \theta_{K}, 0,1\right\rangle \vdash^{*}\left\langle\theta^{\prime}, \downarrow\right.$, [], $\left.,, \theta_{K}^{\prime}, n+1, w\right\rangle$. Hence, $\mathbf{S C}_{C_{1} ; C_{2}}^{\sigma}\left(\theta, n^{\prime}\right)=0$ for all $n^{\prime}>n+1$, and so $\mathbf{S C}_{C_{1} ; C_{2}}^{\sigma}(\theta)=0$.

- If $\mathbf{O}_{C_{1}}^{\sigma}\left(\pi_{L}(\theta)\right)=\uparrow$, then $R H S=\mathbf{S C}_{C_{1}}^{\sigma}\left(\pi_{L}(\theta)\right)$ and for all $k$, we have $\left\langle\pi_{L}(\theta)\right.$, $\left.C_{1},[], \sigma, \pi_{R}(\theta):: \theta_{K}, 0,1\right\rangle \vdash^{*}\left\langle\theta^{\prime}, C_{1}^{\prime \prime}, K, \sigma^{\prime}, \pi_{R}(\theta):: \theta_{K}^{\prime}, k, w\right\rangle$, where $\left(C_{1}^{\prime \prime}, K\right) \neq$ $(\downarrow, K)$ and $\sigma^{\prime} \neq \downarrow$. Fix $k \geq 0$. We have $\mathbf{S C}_{C_{1}}^{\sigma}\left(\pi_{L}(\theta), k\right)=w$ and by Lemma 25. $\left\langle\theta, C_{1} ; C_{2}\right.$, [], $\left.\sigma, \theta_{K}, 0,1\right\rangle \vdash^{*}\left\langle\theta^{\prime}, C_{1}^{\prime \prime}, K @\left[C_{2}\right], \sigma^{\prime}, \theta_{K}^{\prime}, k+1, w\right\rangle$, which implies $\mathbf{S C}_{C_{1} ; C_{2}}^{\sigma}(\theta, k+1)=w$. Hence, $\mathbf{S C}_{C_{1} ; C_{2}}^{\sigma}(\theta, k+1)=\mathbf{S C}_{C_{1}}^{\sigma}\left(\pi_{L}(\theta), k\right)$. Thus,

$$
\begin{aligned}
\mathbf{S C}_{C_{1} ; C_{2}}^{\sigma}(\theta) & =\lim _{n \rightarrow \infty} \mathbf{S C}_{C_{1} ; C_{2}}^{\sigma}(\theta, n) \\
& =\lim _{k \rightarrow \infty} \mathbf{S C}_{C_{1} ; C_{2}}^{\sigma}(\theta, k+1) \\
& =\lim _{k \rightarrow \infty} \mathbf{S C}_{C_{1}}^{\sigma}\left(\pi_{L}(\theta), k\right) \\
& =\mathbf{S C}_{C_{1}}^{\sigma}\left(\pi_{L}(\theta)\right)
\end{aligned}
$$

as required.

Restatement of Proposition 1 If $C_{1} \neq C_{1}^{\prime} ; C_{2}^{\prime}$, then $\mathbf{O}_{C_{1} ; C_{2}}^{\sigma}(\theta)=$ $\mathbf{O}_{C_{2}}^{\mathbf{O}_{C_{1}}^{\sigma}\left(\pi_{L}(\theta)\right)}\left(\pi_{R}(\theta)\right)$ and $\mathbf{S C}_{C_{1} ; C_{2}}^{\sigma}(\theta)=\mathbf{S C}_{C_{1}}^{\sigma}\left(\pi_{L}(\theta)\right) \cdot \mathbf{S C}_{C_{2}}^{\mathbf{O}_{C_{1}}^{\sigma}\left(\pi_{L}(\theta)\right)}\left(\pi_{R}(\theta)\right)$

Proof. This is a combination of Lemma 30 and Lemma 31

Proposition 1 is not applicable when $C_{1}$ is not a sequence of statements, as we cannot know what part of the entropy $\theta$ will be used in the evaluation of which expression without knowing the length of the statement list in $C_{1}$. However, the above result can be generalised using finite shuffling functions, as defined by [39].

Definition 30 ([39] $]$ ).

- A path is a function $\left[d_{1}, \ldots, d_{n}\right]: \mathbb{S} \rightarrow \mathbb{S}$ parametrised by a list of directions $d_{1}, \ldots, d_{n} \in\{L, R\}$, such that $\left[d_{1}, \ldots, d_{n}\right](\theta)=\left(\pi_{d_{1}} \circ \ldots \circ \pi_{d_{n}}\right)(\theta)$.

- $A$ finite shuffling function (FSF) is a function $\phi: \mathbb{S} \rightarrow \mathbb{S}$ such that either $\phi$ is a path or $\phi(\theta)=\phi_{1}(\theta):: \phi_{2}(\theta)$, where $\phi_{1}$ and $\phi_{2}$ are FSFs.

- A sequence of paths is non-duplicating if no path in the sequence is a suffix of another path.

- A FSF $\phi$ is non-duplicating if the sequence of all paths appearing in its definition is non-duplicating.

The following key result shows that entropy rearrangements via FSFs have no effect under integration:

Lemma 32 ([39], Th. 7.6). Any non-duplicating FSF $\phi$ is measure-preserving, i.e., for any measurable $g: \mathbb{S} \rightarrow \overline{\mathbb{R}}_{+}$:

$$
\int g(\phi(\theta)) \mu(d \theta)=\int g(\theta) \mu(d \theta) .
$$

${ }^{9}$ The result in [39] considers $g$ with co-domain $[0, \infty)$ rather than $\overline{\mathbb{R}}_{+}$. It is however, not difficult to check that their result extends to the latter case. 
We now have everything in place to define a version of Proposition 1 for an arbitrary split of a sequencing statement:

Proposition 2 (Sequencing for final states). If $C=C_{1} ; C_{2}$, there exists a non-duplicating FSF $\psi$ such that:

$\mathbf{O}_{C}^{\sigma}(\theta)=\mathbf{O}_{C_{2}}^{\tau}\left(\pi_{R}(\psi(\theta))\right) \quad$ and $\quad \mathbf{S C}_{C}^{\sigma}(\theta)=\mathbf{S C}_{C_{1}}^{\sigma}\left(\pi_{L}(\psi(\theta))\right) \cdot \mathbf{S C}_{C_{2}}^{\tau}\left(\pi_{R}(\psi(\theta))\right)$ with $\tau$ denoting $\mathbf{O}_{C_{1}}^{\sigma}\left(\pi_{L}(\psi(\theta))\right)$.

Proof. By induction on the structure of $C$.

- Base case: $C_{1} \neq C_{1}^{\prime} ; C_{1}^{\prime \prime}$ : the equality holds trivially for $\psi=I d$ by Lemma 30 .

- Induction step: If $C_{1}$ is a sequence of statements, then $C_{1}=C_{1}^{\prime} ; C_{1}^{\prime \prime}$ for some $C_{1}^{\prime}$ such that $C_{1}^{\prime} \neq \hat{C}_{1}^{\prime} \hat{C}_{1}^{\prime \prime}$.

We have:

$$
\begin{aligned}
& \mathbf{O}_{C_{1}^{\prime} ; C_{1}^{\prime \prime} ; C_{2}}^{\sigma}(\theta)_{\text {(by Lemma[3] }}=\mathbf{O}_{C_{1}^{\prime \prime} ; C_{2}}^{\mathbf{O}_{C_{1}^{\prime}}^{\sigma}\left(\pi_{L}(\theta)\right)}\left(\pi_{R}(\theta)\right)
\end{aligned}
$$

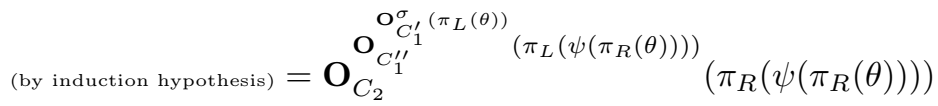

for some non-duplicating FSF $\psi$.

Thus, if $\theta=\theta_{1}:: \theta_{2}$, then

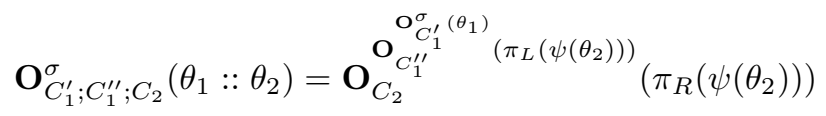

Now, take $\hat{\psi}$ such that $\hat{\psi}\left(\theta_{1}:: \theta_{2}\right)=\left(\theta_{1}:: \pi_{L}\left(\psi\left(\theta_{2}\right)\right)\right):: \pi_{R}\left(\psi\left(\theta_{2}\right)\right)$.

Then

$$
\begin{aligned}
& \mathbf{O}_{C_{2}}^{\mathbf{O}_{C_{1}^{\prime} ; C_{1}^{\prime \prime}}^{\sigma}\left(\pi_{L}\left(\hat{\psi}\left(\theta_{1}:: \theta_{2}\right)\right)\right)}\left(\pi_{R}\left(\hat{\psi}\left(\theta_{1}:: \theta_{2}\right)\right)\right)=\mathbf{O}_{C_{2}}^{\mathbf{O}_{C_{1}^{\prime} ; C_{1}^{\prime \prime}}^{\sigma}\left(\theta_{1}:: \pi_{L}\left(\psi\left(\theta_{2}\right)\right)\right)}\left(\pi_{R}\left(\psi\left(\theta_{2}\right)\right)\right)
\end{aligned}
$$

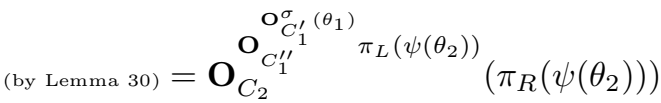

$$
\begin{aligned}
& =\mathbf{O}_{C_{1}^{\prime} ; C_{1}^{\prime \prime} ; C_{2}}^{\sigma}\left(\theta_{1}:: \theta_{2}\right)
\end{aligned}
$$

as required.

For SC, we have:

$$
\begin{aligned}
\mathbf{S C}_{C_{1}^{\prime} ; C_{1}^{\prime \prime} ; C_{2}}^{\sigma}(\theta)_{\text {(by Lemma } 31}= & \mathbf{S C}_{C_{1}^{\prime}}\left(\pi_{L}(\theta)\right) \mathbf{S C}_{C_{1}^{\prime \prime} ; C_{2}}^{\mathbf{O}_{C^{\prime}}^{\sigma}\left(\pi_{L}(\theta)\right)}\left(\pi_{R}(\theta)\right) \\
\text { (by induction hypothesis) } & =\mathbf{S C}_{C_{1}^{\prime}}\left(\pi_{L}(\theta)\right) \mathbf{S C}_{C_{1}^{\prime \prime}}^{\mathbf{O}_{C^{\prime}}^{\prime}\left(\pi_{L}(\theta)\right)}\left(\pi_{L}\left(\psi\left(\pi_{R}(\theta)\right)\right)\right) \\
& \mathbf{S C}_{C_{2}}^{\mathbf{O}_{C_{1}^{\prime \prime}}^{\sigma}\left(\pi_{L}(\theta)\right)}{ }^{\left(\pi_{L}\left(\psi\left(\pi_{R}(\theta)\right)\right)\right)}\left(\pi_{R}\left(\psi\left(\pi_{R}(\theta)\right)\right)\right)
\end{aligned}
$$


for the same $\psi$. Thus, for $\hat{\psi}$ defined above, we have:

$$
\begin{aligned}
& \mathbf{S C}_{C_{1}^{\prime} ; C_{1}^{\prime \prime}}\left(\pi_{L}\left(\hat{\psi}\left(\theta_{1}:: \theta_{2}\right)\right)\right) \mathbf{S C}_{C_{2}}^{\mathbf{O}_{C_{1}^{\prime} ; C_{1}^{\prime \prime}}^{\sigma}\left(\pi_{L}\left(\hat{\psi}\left(\theta_{1}:: \theta_{2}\right)\right)\right)}\left(\pi_{R}\left(\hat{\psi}\left(\theta_{1}:: \theta_{2}\right)\right)\right) \\
= & \mathbf{S C}_{C_{1}^{\prime} ; C_{1}^{\prime \prime}}\left(\theta_{1}:: \pi_{L}\left(\psi\left(\theta_{2}\right)\right)\right) \mathbf{S C}_{C_{2}}^{\mathbf{O}_{C_{1}^{\prime} ; C_{1}^{\prime \prime}}^{\sigma}\left(\theta_{1}:: \pi_{L}\left(\psi\left(\theta_{2}\right)\right)\right)}{ }_{\pi_{R}\left(\psi\left(\theta_{2}\right)\right)}{ }^{\left(^{*}\right)=} \mathbf{S C}_{C_{1}^{\prime}}\left(\theta_{1}\right) \mathbf{S C}_{C_{1}^{\prime \prime}}^{\mathbf{O}_{C_{1}^{\prime}}^{\sigma}\left(\theta_{1}\right)}\left(\pi_{L}\left(\psi\left(\theta_{2}\right)\right)\right) \mathbf{S C}_{C_{2}}^{\mathbf{O}_{C_{1}^{\prime \prime}}^{\sigma}\left(\theta_{1}\right)}\left(\pi_{L}\left(\psi\left(\theta_{2}\right)\right)\right) \\
= & \mathbf{S C}_{C_{1}^{\prime} ; C_{1}^{\prime \prime} ; C_{2}}^{\sigma}\left(\theta_{1}:: \theta_{2}\right)
\end{aligned}
$$

as required, where the equality $(*)$ follows from Lemmas 30 and 31 .

Now we only need to show that $\hat{\psi}$ is a non-duplicating FSF.

First, let us show that $\hat{\psi}$ is indeed a FSF. To this end, we need to show that if $\psi$ is a FSF, then $\psi^{\prime}(\theta)=\psi\left(\pi_{R}(\theta)\right)$ is also a FSF. We prove this by induction on the structure of $\psi$ :

- Base case: if $\psi$ is a path $\left[d_{1}, \ldots, d_{n}\right]$, then $\psi \circ \pi_{R}$ is the path $\left[d_{1}, \ldots, d_{n}, R\right]$, so it is a FSF.

- Induction step: Suppose that $\psi(\theta)=\psi_{1}(\theta):: \psi_{2}(\theta)$ and that $\psi_{1} \circ \pi_{R}$ and $\psi_{2} \circ \pi_{R}$ are FSFs. Then we have $\psi\left(\pi_{R}(\theta)\right)=\psi_{1}\left(\pi_{R}(\theta)\right):: \psi_{2}\left(\pi_{R}(\theta)\right)=$ $\left(\psi_{1} \circ \pi_{R}\right)(\theta)::\left(\psi_{2} \circ \pi_{R}\right)(\theta)$, so $\psi \circ \pi_{R}$ is a FSF by definition.

Now, we show that $\psi^{\prime \prime}(\theta)=\pi_{L}\left(\psi\left(\pi_{R}(\theta)\right)\right)=\pi_{L}\left(\psi^{\prime}(\theta)\right)$ is a FSF: if $\psi^{\prime}$ is a path $\left[d_{1}, \ldots, d_{n}\right]$, then $\psi^{\prime \prime}$ is a path $\left[L, d_{1}, \ldots, d_{n}\right]$, and if $\psi^{\prime}=\psi_{1}^{\prime}:: \psi_{2}^{\prime}$, then $\pi_{L}\left(\psi^{\prime}(\theta)\right)=\pi_{L}\left(\psi_{1}^{\prime}(\theta):: \psi_{2}^{\prime}(\theta)\right)=\psi_{1}^{\prime}(\theta)$. Similarly, we can show that $\pi_{R}\left(\psi\left(\pi_{R}(\theta)\right)\right)$ is a FSF. Hence, $\hat{\psi}$ is a FSF by definition.

Finally, we need to show that $\hat{\psi}$ is non-duplicating.

We can show by a simple induction that for any $\psi$, the set of paths $\mathcal{P}_{\psi \circ \pi_{R}}$ in $\psi \circ \pi_{R}$ is $\left\{p R \mid p \in \mathcal{P}_{\psi}\right\}$, where $\mathcal{P}_{\psi}$ is the set of paths in $\psi$ and juxtaposition denotes concatenation.

If $\psi$ is a path $p$, then $\pi_{L} \circ \psi \circ \pi_{R}$ and $\pi_{R} \circ \psi \circ \pi_{R}$ are paths $L p R$ and $R p R$. Hence, the set of paths in $\hat{\psi}$ is $\{[L], L p R, R p R\}$. It is instantly clear that no path is a suffix of another, so $\hat{\psi}$ is non-duplicating.

If $\psi(\theta)=\psi_{1}(\theta):: \psi_{2}(\theta)$, then $\left(\pi_{L} \circ \psi \circ \pi_{R}\right)(\theta)=\pi_{L}\left(\psi_{1}\left(\pi_{R}(\theta)\right):: \psi_{2}\left(\pi_{R}(\theta)\right)\right)=$ $\psi_{1}\left(\pi_{R}(\theta)\right)$, so the set of paths in $\pi_{L} \circ \psi \circ \pi_{R}$ is $\left\{p R \mid p \in \mathcal{P}_{\psi_{1}}\right\}$, where $\mathcal{P}_{\psi_{1}}$ is the set of paths in $\psi_{1}$. Similarly, the set of paths in $\pi_{R} \circ \psi \circ \pi_{R}$ is $\left\{p R \mid p \in \mathcal{P}_{\psi_{2}}\right\}$, where $\mathcal{P}_{\psi_{2}}$ is the set of paths in $\psi_{2}$. Since $\mathcal{P}_{\psi}=\mathcal{P}_{\psi_{1}} \cup \mathcal{P}_{\psi_{2}}$, the set of paths in the entire definition of $\hat{\psi}$ is $\{[L]\} \cup\left\{p R \mid p \in \mathcal{P}_{\psi}\right\}$. It is clear that $[L]$ is not a suffix of any path of the form $p R$ (as all such paths end with $R$ ). Moreover, if there were paths $p_{1}, p_{2} \in \mathcal{P}_{\psi}$ such that $p_{1} R$ was a suffix of $p_{2} R$, then $p_{1}$ would be a suffix of $p_{2}$, which would contradict the assumption.

Hence, $\hat{\psi}$ is non-duplicating, which ends the proof. 


\section{D.3 Approximating while-loops}

To simplify reasoning about while-loops, it is useful - and common in program semantics - to consider finite approximations of loops in which the maximal number of iterations is bounded. To that end, we define the $n$-th unfolding of a guarded loop inductively as follows:

$$
\begin{aligned}
\text { while }^{0}(\phi)\{C\} & =\text { diverge } \\
\text { while }^{n+1}(\phi)\{C\} & =\operatorname{if}(\phi)\left\{C ; \text { while }^{n}(\phi)\{C\}\right\} .
\end{aligned}
$$

In the limit, bounded while-loops behave as standard while-loops. We use this result to define the evaluation of measurable function $f$ on successful termination states of a while-loop, scaled by its score as a limit of approximations. As we are interested in $f$ on proper states, we use $\hat{f}$ rather than $f$.

Proposition 3. Let loop $C=$ while $(\phi)\left\{C^{\prime}\right\}$ and $C^{n}=$ while ${ }^{n}(\phi)\left\{C^{\prime}\right\}$ its $n$-th approximation. Then:

$$
\hat{f}\left(\mathbf{O}_{C}^{\sigma}(\theta)\right) \cdot \mathbf{S C}_{C}^{\sigma}(\theta)=\sup _{n} \hat{f}\left(\mathbf{O}_{C^{n}}^{\sigma}(\theta)\right) \cdot \mathbf{S C}_{C^{n}}^{\sigma}(\theta) .
$$

The following monotonicity property is relevant later when proving the relationship between the operational semantics of PL and its denotational semantics. As before let $C^{n}=$ while ${ }^{n}(\phi)\left\{C^{\prime}\right\}$.

Proposition 4. If $n \geq k$ and $\hat{f}(\xi)=\hat{f}(\uparrow)=0$, then $\hat{f}\left(\mathbf{O}_{C^{n}}^{\sigma}(\theta)\right) \cdot \mathbf{S C}_{C^{n}}^{\sigma}(\theta) \geq$ $\hat{f}\left(\mathbf{O}_{C^{k}}^{\sigma}(\theta)\right) \cdot \mathbf{S C}_{C^{k}}^{\sigma}(\theta)$.

Similarly, we want to show that the sequence $\hat{f}\left(\mathbf{O}_{C^{n}}^{\sigma}(\theta)\right) \cdot \mathbf{S C}_{C^{n}}^{\sigma}(\theta)$ approximates $\check{f}\left(\mathbf{O}_{C}^{\sigma}(\theta)\right) \cdot \mathbf{S C}_{C}^{\sigma}(\theta)$. This result allows us to express the anticipated value of the function $\hat{f}$ for a given fixed entropy as a limit of approximations, and by integrating both sides with respect to the measure on entropies we get that the expected value of $\hat{f}$ can also be expressed as a limit of approximations. We will use this result in the proof of Theorem 2, Recall that $\check{f}(\tau)=1$ for $\tau=\uparrow$.

Proposition 5. Let loop $C=$ while $(\phi)\left\{C^{\prime}\right\}$ and $C^{n}=$ while $(\phi)\left\{C^{\prime}\right\}$ its $n$-th approximation. Take a function $f \leq 1$. Then

$$
\check{f}\left(\mathbf{O}_{C}^{\sigma}(\theta)\right) \cdot \mathbf{S C}_{C}^{\sigma}(\theta)=\inf _{n} \check{f}\left(\mathbf{O}_{C^{n}}^{\sigma}(\theta)\right) \cdot \mathbf{S C}_{C^{n}}^{\sigma}(\theta) .
$$

Proposition 6. If $n \geq k$ and $f \leq 1$, then

$$
\check{f}\left(\mathbf{O}_{C^{n}}^{\sigma}(\theta)\right) \cdot \mathbf{S C}_{C^{n}}^{\sigma}(\theta) \leq \check{f}\left(\mathbf{O}_{C^{k}}^{\sigma}(\theta)\right) \cdot \mathbf{S C}_{C^{k}}^{\sigma}(\theta) .
$$

The rest of this section is the proof of Propositions 3 , 4, 5 and 6, which will be needed to prove the case of while-loops in Theorem 1 and Theorem 2 , The first key fact that we want to show is that for non-diverging executions, a bounded while-loop of the form while ${ }^{n}(\phi)\{C\}$ behaves just like while $(\phi)\{C\}$ for a sufficiently large $n$. We formalise and prove it using two auxiliary relations on configurations. 
Replacing while $(\phi)\{C\}$ with while ${ }^{n}(\phi)\{C\}$ We first prove that in all nondivering configurations, if the expression is of the form while $(\phi)\{C\}$, we can replace it with while ${ }^{n}(\phi)\{C\}$ for a large enough $n$, without changing the final configuration reached after reduction is completed. To this end, we first define an indexed relation $\left(\sim^{n}\right)$ on configurations. We begin with auxiliary relations $C \sim^{n} C^{\prime}$ and $K \sim^{n} K^{\prime}$, defined inductively as follows:

$$
C \sim{ }^{0} C^{\prime}
$$

For $n>0$ :

\begin{tabular}{l}
$\frac{C \sim^{n} C}{\downarrow \sim^{n} \downarrow}$ \\
$\frac{k \geq n}{\operatorname{while}(\phi)\{C\} \sim^{n} \text { while }^{k}(\phi)\{C\}}$ \\
$k \geq n$ \\
\hline while $(\phi)\{C\} \sim^{n}$ while $(\phi)\{C\}$ \\
$\frac{k \geq n \quad l \geq n}{\text { while }(\phi)\{C\} \sim^{n} \text { while }(\phi)\{C\}}$ \\
$\frac{C_{2} \sim^{n} C_{2}^{\prime}}{C_{1} ; C_{2} \sim^{n} C_{1} ; C_{2}^{\prime}}$ \\
$\frac{\forall i \in 1 . . n \quad C_{i} \sim^{n} C_{i}^{\prime}}{\left[C_{1}, \ldots, C_{n}\right] \sim^{n}\left[C_{1}^{\prime}, \ldots, C_{n}^{\prime}\right]}$
\end{tabular}

We then naturally extend the definition to configurations:

$$
\left\langle\theta, C, K, \sigma, \theta_{K}, m, w\right\rangle \sim^{0}\left\langle\theta^{\prime}, C^{\prime}, K^{\prime}, \sigma^{\prime}, \theta_{K}^{\prime}, m^{\prime}, w^{\prime}\right\rangle
$$

For $n>0$ :

$\frac{C \sim^{n} C^{\prime} K \sim^{n} K^{\prime}}{\left\langle\theta, C, K, \sigma, \theta_{K}, m, w\right\rangle \sim^{n}\left\langle\theta, C^{\prime}, K^{\prime}, \sigma, \theta_{K}, m, w\right\rangle}$

We can immediately check that if two configurations are related by $\left(\sim^{n}\right)$ for some $n>0$, then if we perform one step of reductions on both of them, the resulting configurations are guaranteed to be related at least by $\left(\sim^{n-1}\right)$.

Lemma 33. $\sim^{n}$ is a stratified bisumulation-that is, $\left\langle\theta, C, K, \sigma, \theta_{K}, m, w\right\rangle \sim^{0}$ $\left\langle\theta^{\prime}, C^{\prime}, K^{\prime}, \sigma^{\prime}, \theta_{K}^{\prime}, m^{\prime}, w^{\prime}\right\rangle$ and for $n>0$ :

- if $\left\langle\theta, C, K, \sigma, \theta_{K}, m, w\right\rangle \sim^{n}\left\langle\theta, C^{\prime}, K^{\prime}, \sigma, \theta_{K}, m, w\right\rangle$ and $\left\langle\theta, C, K, \sigma, \theta_{K}, m, w\right\rangle \vdash\left\langle\theta^{\prime \prime}, C^{\prime \prime}, K^{\prime \prime}, \sigma^{\prime \prime}, \theta_{K}^{\prime \prime}, m+1, w^{\prime \prime}\right\rangle$, then $\left\langle\theta, C^{\prime}, K^{\prime}, \sigma\right.$, $\left.\theta_{K}, m, w\right\rangle \vdash\left\langle\theta^{\prime \prime}, C^{\prime \prime \prime}, K^{\prime \prime \prime}, \sigma^{\prime \prime}, \theta_{K}^{\prime \prime}, m+1, w^{\prime \prime}\right\rangle$ and $\left\langle\theta^{\prime \prime}, C^{\prime \prime}, K^{\prime \prime}, \sigma^{\prime \prime}, \theta_{K}^{\prime \prime}, m+1\right.$, $\left.w^{\prime \prime}\right\rangle \sim^{n-1}\left\langle\theta^{\prime \prime}, C^{\prime \prime \prime}, K^{\prime \prime \prime}, \sigma^{\prime \prime}, \theta_{K}^{\prime \prime}, m+1, w^{\prime \prime}\right\rangle$

- if $\left\langle\theta, C, K, \sigma, \theta_{K}, m, w\right\rangle \sim^{n}\left\langle\theta, C^{\prime}, K^{\prime}, \sigma, \theta_{K}, m, w\right\rangle$ and $\left\langle\theta, C^{\prime}, K^{\prime}, \sigma, \theta_{K}, m, w\right\rangle \vdash\left\langle\theta^{\prime \prime}, C^{\prime \prime \prime}, K^{\prime \prime \prime}, \sigma^{\prime \prime}, \theta_{K}^{\prime \prime}, m+1, w^{\prime \prime}\right\rangle$, then $\langle\theta, C, K, \sigma$, $\left.\theta_{K}, m, w\right\rangle \vdash\left\langle\theta^{\prime \prime}, C^{\prime \prime}, K^{\prime \prime}, \sigma^{\prime \prime}, \theta_{K}^{\prime \prime}, m+1, w^{\prime \prime}\right\rangle$ and $\left\langle\theta^{\prime \prime}, C^{\prime \prime}, K^{\prime \prime}, \sigma^{\prime \prime}, \theta_{K}^{\prime \prime}, m+1\right.$, $\left.w^{\prime \prime}\right\rangle \sim^{n-1}\left\langle\theta^{\prime \prime}, C^{\prime \prime \prime}, K^{\prime \prime \prime}, \sigma^{\prime \prime}, \theta_{K}^{\prime \prime}, m+1, w^{\prime \prime}\right\rangle$

Proof. By inspection. 
This result naturally generalises to multi-step reduction.

Corollary 8. If $\left\langle\theta, C, K, \sigma, \theta_{K}, m, w\right\rangle \sim^{n}\left\langle\theta, C^{\prime}, K^{\prime}, \sigma, \theta_{K}, m, w\right\rangle$ and $\langle\theta, C, K$, $\left.\sigma, \theta_{K}, m, w\right\rangle \vdash^{*}\left\langle\theta^{\prime \prime}, C^{\prime \prime}, K^{\prime \prime}, \sigma^{\prime \prime}, \theta_{K}^{\prime \prime}, m+n^{\prime}, w^{\prime \prime}\right\rangle$ and $n^{\prime}<n$ then $\left\langle\theta, C^{\prime}, K^{\prime}, \sigma\right.$, $\left.\theta_{K}, m, w\right\rangle \vdash^{*}\left\langle\theta^{\prime \prime}, C^{\prime \prime \prime}, K^{\prime \prime \prime}, \sigma^{\prime \prime}, \theta_{K}^{\prime \prime}, m+n^{\prime}, w^{\prime \prime}\right\rangle$ and $\left\langle\theta^{\prime \prime}, C^{\prime \prime}, K^{\prime \prime}, \sigma^{\prime \prime}, \theta_{K}^{\prime \prime}, m+n^{\prime}\right.$, $\left.w^{\prime \prime}\right\rangle \sim^{n-n^{\prime}}\left\langle\theta^{\prime \prime}, C^{\prime \prime \prime}, K^{\prime \prime \prime}, \sigma^{\prime \prime}, \theta_{K}^{\prime \prime}, m+n^{\prime}, w^{\prime \prime}\right\rangle$ (and vice versa).

This leads us to the desired result for terminating runs.

Lemma 34. If $\left\langle\theta\right.$, while $(\phi)\{C\}$, [], $\left.\sigma, \theta_{K}, n, w\right\rangle \vdash^{*}\left\langle\theta^{\prime}, \downarrow\right.$, [], $\left.\sigma^{\prime}, \theta_{K}, n+n^{\prime}, w^{\prime}\right\rangle$, then there exists $k$ such that $\left\langle\theta\right.$, while $\left.{ }^{k}(\phi)\{C\},[], \sigma, \theta_{K}, n, w\right\rangle \vdash^{*}\left\langle\theta^{\prime}, \downarrow,[], \sigma^{\prime}, \theta_{K}\right.$, $\left.n+n^{\prime}, w^{\prime}\right\rangle$

Proof. Take $k=n^{\prime}+1$. We clearly have while $(\phi)\{C\} \sim^{n^{\prime}+1}$ while ${ }^{n^{\prime}+1}(\phi)\{C\}$, and so $\left\langle\theta\right.$, while $(\phi)\{C\}$, [], $\left.\sigma, \theta_{K}, n, w\right\rangle \sim^{n^{\prime}+1}\left\langle\theta\right.$, while $\operatorname{en}^{n^{\prime}+1}(\phi)\{C\},[], \sigma, \theta_{K}, n$, $w\rangle$. By Corollary [8, $\left\langle\theta\right.$, while $n^{n^{\prime}+1}(\phi)\{C\}$, [], $\left.\sigma, \theta_{K}, n, w\right\rangle \vdash^{*}\left\langle\theta^{\prime}, C^{\prime}, K^{\prime}, \sigma^{\prime}, \theta_{K}\right.$, $\left.n+n^{\prime}, w^{\prime}\right\rangle$, where $\downarrow \sim^{1} C^{\prime}$ and [] $\sim^{1} K^{\prime}$, which implies $C^{\prime}=\downarrow$ and $K^{\prime}=$ []. Thus, the statement always holds for $k=n^{\prime}+1$.

This result leads to the following statement about the $\mathbf{O}_{C}^{\sigma}$ and $\mathbf{S} \mathbf{C}_{C}^{\sigma}$ functions:

Lemma 35. For each $\phi, C, \sigma, \theta$, such that $\mathbf{O}_{\text {while }(\phi)\{C\}}^{\sigma}(\theta) \in \Omega_{\sigma}$ there is a $k$ such that $\mathbf{O}_{\text {while }(\phi)\{C\}}^{\sigma}(\theta)=\mathbf{O}_{\text {while }}^{\sigma}(\phi)\{C\}(\theta)$ and $\mathbf{S C}_{\text {while }(\phi)\{C\}}^{\sigma}(\theta)=$ $\mathbf{S C}_{\text {while }}^{\sigma}(\phi)\{C\}(\theta)$

Proof. If $\mathbf{O}_{\text {while }(\phi)\{C\}}^{\sigma}(\theta) \in \Omega_{\sigma}$, then by definition of $\mathbf{O},\langle\theta$, while $(\phi)\{C\},[], \sigma$, $\left.\theta_{K}, 0,1\right\rangle \vdash^{*}\left\langle\theta^{\prime}, \downarrow,[], \sigma^{\prime}, \theta_{K}^{\prime}, n, w\right\rangle$, where $\sigma^{\prime} \neq \downarrow$. This implies $\mathbf{O}_{\text {while }(\phi)\{C\}}^{\sigma}(\theta)=$ $\sigma^{\prime}$ and $\mathbf{S C}_{\text {while }(\phi)\{C\}}^{\sigma}(\theta)=w$. By Lemma 34, there is a $k$ such that $\langle\theta$, while $\left.^{k}(\phi)\{C\},[], \sigma, \theta_{K}, 0,1\right\rangle \vdash^{*}\left\langle\theta^{\prime}, \downarrow,[], \sigma^{\prime}, \theta_{K}^{\prime}, n, w\right\rangle$. Thus, $\mathbf{O}_{\text {while }^{k}(\phi)\{C\}}^{\sigma}(\theta)=$

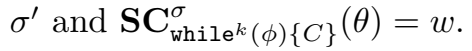

We can also show that if the evaluation of while $(\phi)\{C\}$ gets stuck, so does the evaluation of while $\mathrm{e}^{k}(\phi)\{C\}$ for large enough $k$.

Lemma 36. If $\left\langle\theta\right.$, while $(\phi)\{C\}$, [], $\left.\sigma, \theta_{K}, n, w\right\rangle \vdash^{*}\left\langle\theta^{\prime}, C^{\prime}, K, \sigma^{\prime}, \theta_{K}^{\prime}, n+n^{\prime}, w^{\prime}\right\rangle \nvdash$, then there exists $k$ such that $\left\langle\theta\right.$, while $\left.{ }^{k}(\phi)\{C\},[], \sigma, \theta_{K}, n, w\right\rangle \vdash^{*}\left\langle\theta^{\prime}, C^{\prime \prime}, K^{\prime}, \sigma^{\prime}\right.$, $\left.\theta_{K}^{\prime}, n+n^{\prime}, w^{\prime}\right\rangle \nvdash$.

Proof. Again, take $k=n^{\prime}+1$. We have while $(\phi)\{C\} \sim^{n^{\prime}+1}$ while ${ }^{n^{\prime}+1}(\phi)\{C\}$, and so $\left\langle\theta\right.$, while $(\phi)\{C\}$, [], $\left.\sigma, \theta_{K}, n, w\right\rangle \sim^{n^{\prime}+1}\left\langle\theta\right.$, while ${ }^{n^{\prime}+1}(\phi)\{C\}$, [], $\sigma, \theta_{K}, n$, $w\rangle$. By Corollary [8, $\left\langle\theta\right.$, while $\left.{ }^{n^{\prime}+1}(\phi)\{C\},[], \sigma, \theta_{K}, n, w\right\rangle \vdash^{*}\left\langle\theta^{\prime}, C^{\prime \prime}, K^{\prime}, \sigma^{\prime}, \theta_{K}\right.$, $\left.n+n^{\prime}, w^{\prime}\right\rangle$, where $C^{\prime} \sim^{1} C^{\prime \prime}$ and $K \sim^{1} K^{\prime}$. By case analysis on the derivation of $C^{\prime} \sim^{1} C^{\prime \prime}$, and using the fact that $K$ and $K^{\prime}$ must have the same length, we conclude that $\left\langle\theta^{\prime}, C^{\prime}, K, \sigma^{\prime}, \theta_{K}^{\prime}, n+n^{\prime}, w^{\prime}\right\rangle$ reduces if and only if $\left\langle\theta^{\prime}, C^{\prime \prime}, K^{\prime}, \sigma^{\prime}\right.$, $\left.\theta_{K}^{\prime}, n+n^{\prime}, w^{\prime}\right\rangle$ reduces. 
Replacing while ${ }^{n}(\phi)\{C\}$ with while $(\phi)\{C\}$ We now prove the converse to the above result- that if while $\mathrm{e}^{n}(\phi)\{C\}$ evaluates with some entropy $\theta$, the unbounded loop while $(\phi)\{C\}$ evaluates to the same configuration. We begin with another relation $\unlhd$ on configurations, which effectively states that for two configurations $\kappa_{1}$ and $\kappa_{2}$, if $\kappa_{1} \unlhd \kappa_{2}$ and $\kappa_{1}$ evaluates, then $\kappa_{2}$ is guaranteed to evaluate to the same final configuration. This relation is defined inductively as follows:

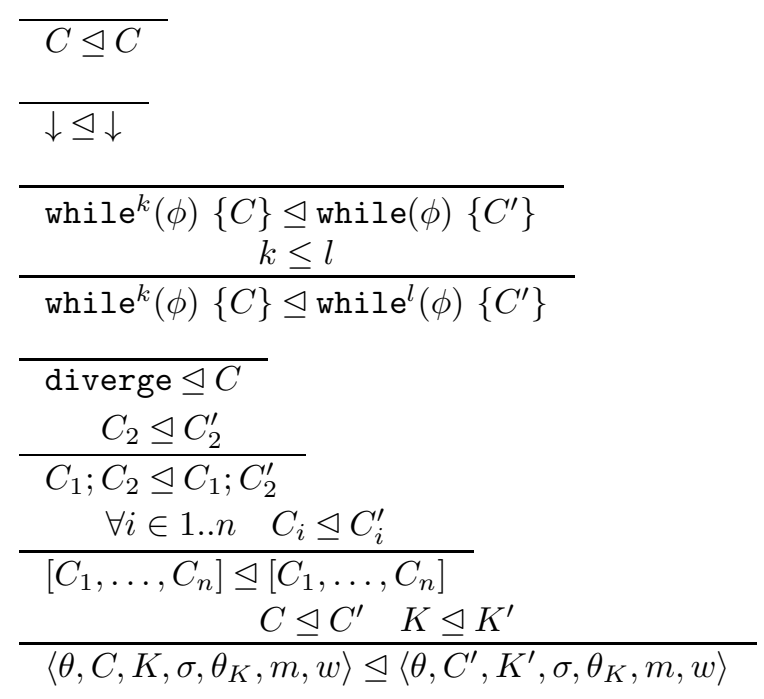

Lemma 37. $\unlhd$ is a simulation-that is, if $\left\langle\theta, C, K, \sigma, \theta_{K}, m, w\right\rangle \unlhd\left\langle\theta, C^{\prime}, K^{\prime}, \sigma\right.$, $\left.\theta_{K}, m, w\right\rangle$ and $\left\langle\theta, C, K, \sigma, \theta_{K}, m, w\right\rangle \vdash\left\langle\theta^{\prime \prime}, C^{\prime \prime}, K^{\prime \prime}, \sigma^{\prime \prime}, \theta_{K}^{\prime \prime}, m+1, w^{\prime \prime}\right\rangle$ and $C \neq$ diverge, then $\left\langle\theta, C^{\prime}, K^{\prime}, \sigma, \theta_{K}, m, w\right\rangle \vdash\left\langle\theta^{\prime \prime}, C^{\prime \prime \prime}, K^{\prime \prime \prime}, \sigma^{\prime \prime}, \theta_{K}^{\prime \prime}, m+1, w^{\prime \prime}\right\rangle$ and $\left\langle\theta^{\prime \prime}\right.$, $\left.C^{\prime \prime}, K^{\prime \prime}, \sigma^{\prime \prime}, \theta_{K}^{\prime \prime}, m+1, w^{\prime \prime}\right\rangle \unlhd\left\langle\theta^{\prime \prime}, C^{\prime \prime \prime}, K^{\prime \prime \prime}, \sigma^{\prime \prime}, \theta_{K}^{\prime \prime}, m+1, w^{\prime \prime}\right\rangle$

Proof. By case analysis on the reduction rules.

Corollary 9. If $\left\langle\theta, C, K, \sigma, \theta_{K}, m, w\right\rangle \unlhd\left\langle\theta, C^{\prime}, K^{\prime}, \sigma, \theta_{K}, m, w\right\rangle$ and $\langle\theta, C, K, \sigma$, $\left.\theta_{K}, m, w\right\rangle \vdash^{*}\left\langle\theta^{\prime \prime}, C^{\prime \prime}, K^{\prime \prime}, \sigma^{\prime \prime}, \theta_{K}^{\prime \prime}, m+n^{\prime}, w^{\prime \prime}\right\rangle$ and $C^{\prime \prime} \neq$ diverge, then

$\left\langle\theta, C^{\prime}, K^{\prime}, \sigma, \theta_{K}, m, w\right\rangle \vdash^{*}\left\langle\theta^{\prime \prime}, C^{\prime \prime \prime}, K^{\prime \prime \prime}, \sigma^{\prime \prime}, \theta_{K}^{\prime \prime}, m+n^{\prime}, w^{\prime \prime}\right\rangle$ and $\left\langle\theta^{\prime \prime}, C^{\prime \prime}, K^{\prime \prime}, \sigma^{\prime \prime}\right.$, $\left.\theta_{K}^{\prime \prime}, m+n^{\prime}, w^{\prime \prime}\right\rangle \unlhd\left\langle\theta^{\prime \prime}, C^{\prime \prime \prime}, K^{\prime \prime \prime}, \sigma^{\prime \prime}, \theta_{K}^{\prime \prime}, m+n^{\prime}, w^{\prime \prime}\right\rangle$

We can now show the desired result for terminating reductions.

Lemma 38. If $\left\langle\theta\right.$, $\operatorname{while}^{k}(\phi)\{C\}$, [], $\left.\sigma, \theta_{K}, n, w\right\rangle \vdash^{*}\left\langle\theta^{\prime}, \downarrow\right.$, [], $\left.\sigma^{\prime}, \theta_{K}, n+n^{\prime}, w^{\prime}\right\rangle$, then $\left\langle\theta\right.$, while $\left.(\phi)\{C\},[], \sigma, \theta_{K}, n, w\right\rangle \vdash^{*}\left\langle\theta^{\prime}, \downarrow,[], \sigma^{\prime}, \theta_{K}, n+n^{\prime}, w^{\prime}\right\rangle$.

Proof. We have $\left\langle\theta\right.$, while $\left.{ }^{k}(\phi)\{C\},[], \sigma, \theta_{K}, n, w\right\rangle \unlhd\left\langle\theta\right.$, while $(\phi)\{C\},[], \sigma, \theta_{K}, n$, $w\rangle$, so by Corollary 9, $\left\langle\theta\right.$, while $\left.(\phi)\{C\},[], \sigma, \theta_{K}, n, w\right\rangle \vdash^{*}\left\langle\theta^{\prime}, C^{\prime}, K^{\prime}, \sigma^{\prime}, \theta_{K}, n+n^{\prime}\right.$, $\left.w^{\prime}\right\rangle$ where $\downarrow \unlhd C^{\prime}$ and [] $\unlhd K^{\prime}$, which implies $C^{\prime}=\downarrow$ and $K^{\prime}=[]$.

If the evaluation of while $\mathrm{e}^{k}(\phi)\{C\}$ gets stuck, so does the evaluation of while $(\phi)\{C\}$. 
Lemma 39. If $\left\langle\theta, C, K, \sigma, \theta_{K}, n, w\right\rangle \vdash\left\langle\theta^{\prime}, C^{\prime}, K^{\prime}, \sigma^{\prime}, \theta_{K}^{\prime}, n^{\prime}, w^{\prime}\right\rangle$ and $\hat{C} \unlhd C$ and $\hat{K} \unlhd K$, then $\left\langle\theta, \hat{C}, \hat{K}, \sigma, \theta_{K}, n, w\right\rangle \vdash\left\langle\theta^{\prime \prime}, C^{\prime \prime}, K^{\prime \prime}, \sigma^{\prime \prime}, \theta_{K}^{\prime \prime}, n^{\prime \prime}, w^{\prime \prime}\right\rangle$.

Proof. By case analysis on the derivation of $\hat{C} \unlhd C$.

Lemma 40. If $\left\langle\theta, \operatorname{while}^{k}(\phi)\{C\}\right.$, [], $\left.\sigma, \theta_{K}, n, w\right\rangle \vdash^{*}\left\langle\theta^{\prime}, C^{\prime}, K, \sigma^{\prime}, \theta_{K}^{\prime}, n+n^{\prime}, w^{\prime}\right\rangle \nvdash$, then $\left\langle\theta\right.$, while $(\phi)\{C\}$, [], $\left.\sigma, \theta_{K}, n, w\right\rangle \vdash^{*}\left\langle\theta^{\prime}, C^{\prime \prime}, K^{\prime}, \sigma^{\prime}, \theta_{K}^{\prime}, n+n^{\prime}, w^{\prime}\right\rangle \nvdash$.

Proof. If $C^{\prime} \neq$ diverge, then by Corollary 9, $\left\langle\theta\right.$, while $(\phi)\{C\}$, [], $\left.\sigma, \theta_{K}, n, w\right\rangle \vdash^{*}$ $\left\langle\theta^{\prime}, C^{\prime \prime}, K^{\prime}, \sigma^{\prime}, \theta_{K}, n+n^{\prime}, w^{\prime}\right\rangle$ where $C^{\prime} \unlhd C^{\prime \prime}$ and $K \unlhd K^{\prime}$. By Lemma 39, if $\left\langle\theta^{\prime}\right.$, $\left.C^{\prime \prime}, K^{\prime}, \sigma^{\prime}, \theta_{K}, n+n^{\prime}, w^{\prime}\right\rangle$ reduces, then $\left\langle\theta^{\prime}, C^{\prime}, K, \sigma^{\prime}, \theta_{K}, n+n^{\prime}, w^{\prime}\right\rangle$ also reduces, contradicting the assumption. Hence, $\left\langle\theta^{\prime}, C^{\prime \prime}, K^{\prime}, \sigma^{\prime}, \theta_{K}, n+n^{\prime}, w^{\prime}\right\rangle \nvdash$, as required.

If $C^{\prime}=$ diverge, then $\sigma^{\prime}=\xi$, as otherwise $\left\langle\theta^{\prime}\right.$, diverge, $K, \sigma^{\prime}, \theta_{K}^{\prime}, n+n^{\prime}$, $\left.w^{\prime}\right\rangle$ would reduce by (diverge). However, $\left\langle\theta^{\prime}\right.$, diverge, $\left.K, \downarrow, \theta_{K}^{\prime}, n+n^{\prime}, w^{\prime}\right\rangle$ is not derivable from any initial configuration other than itself. Hence, $n^{\prime}=0$ and $k=0$ and $\sigma=\xi$. Since no configuration with state $\xi$ reduces, we have $\langle\theta$, while $(\phi)\{C\},[], \downarrow$, $\left.\theta_{K}, n, w\right\rangle \nvdash$, as required.

Corollary 10. $\mathbf{O}_{\text {while }(\phi)\{C\}}^{\sigma}(\theta) \geq \mathbf{O}_{\text {while }^{k}(\phi)\{C\}}^{\sigma}(\theta)$ for all $k$.

Replacing one bounded loop with another We now prove that a bounded loop while $\mathrm{e}^{k}(\phi)\{C\}$ can be safely replaced by another bounded loop with a higher bound.

Lemma 41. If $m \geq k$ and $\left\langle\theta\right.$, while ${ }^{k}(\phi)\{C\}$, [], $\left.\sigma, \theta_{K}, n, w\right\rangle \vdash^{*}\left\langle\theta^{\prime}, \downarrow\right.$, [], $\sigma^{\prime}, \theta_{K}$, $\left.n+n^{\prime}, w^{\prime}\right\rangle$, then $\left\langle\theta\right.$, while $\left.{ }^{m}(\phi)\{C\},[], \sigma, \theta_{K}, n, w\right\rangle \vdash^{*}\left\langle\theta^{\prime}, \downarrow,[], \sigma^{\prime}, \theta_{K}, n+n^{\prime}, w^{\prime}\right\rangle$

Proof. We have $\left\langle\theta\right.$, while $\left.{ }^{k}(\phi)\{C\},[], \sigma, \theta_{K}, n, w\right\rangle \unlhd\left\langle\theta\right.$, while ${ }^{m}(\phi)\{C\},[], \sigma, \theta_{K}, n$, $w\rangle$, so by Corollary 9, $\left\langle\theta\right.$, while $\left.{ }^{m}(\phi)\{C\},[], \sigma, \theta_{K}, n, w\right\rangle \vdash^{*}\left\langle\theta^{\prime}, C^{\prime}, K^{\prime}, \sigma^{\prime}, \theta_{K}, n+\right.$ $\left.n^{\prime}, w^{\prime}\right\rangle$ where $\downarrow \unlhd C^{\prime}$ and [] $\unlhd K^{\prime}$, which implies $C^{\prime}=\downarrow$ and $K^{\prime}=[]$.

We show the same property for reductions which get stuck.

Lemma 42. If $m \geq k$ and $\left\langle\theta\right.$, while ${ }^{k}(\phi)\{C\}$, [], $\left.\sigma, \theta_{K}, n, w\right\rangle \vdash^{*}\left\langle\theta^{\prime}, C^{\prime}, K, \sigma^{\prime}, \theta_{K}^{\prime}\right.$, $\left.n+n^{\prime}, w^{\prime}\right\rangle \nvdash$, then $\left\langle\theta\right.$, while $\left.{ }^{m}(\phi)\{C\},[], \sigma, \theta_{K}, n, w\right\rangle \vdash^{*}\left\langle\theta^{\prime}, C^{\prime \prime}, K^{\prime}, \sigma^{\prime}, \theta_{K}^{\prime}, n+n^{\prime}\right.$, $\left.w^{\prime}\right\rangle \nvdash$.

Proof. If $C^{\prime} \neq$ diverge, then by Corollary 9] $\left\langle\theta\right.$, while ${ }^{m}(\phi)\{C\},[], \sigma, \theta_{K}, n$, $w\rangle \vdash^{*}\left\langle\theta^{\prime}, C^{\prime \prime}, K^{\prime}, \sigma^{\prime}, \theta_{K}, n+n^{\prime}, w^{\prime}\right\rangle$ where $C^{\prime} \unlhd C^{\prime \prime}$ and $K \unlhd K^{\prime}$. By Lemma 39., if $\left\langle\theta^{\prime}, C^{\prime \prime}, K^{\prime}, \sigma^{\prime}, \theta_{K}, n+n^{\prime}, w^{\prime}\right\rangle$ reduces, then $\left\langle\theta^{\prime}, C^{\prime}, K, \sigma^{\prime}, \theta_{K}, n+n^{\prime}, w^{\prime}\right\rangle$ also reduces, contradicting the assumption. Hence, $\left\langle\theta^{\prime}, C^{\prime \prime}, K^{\prime}, \sigma^{\prime}, \theta_{K}, n+n^{\prime}, w^{\prime}\right\rangle \nvdash$, as required.

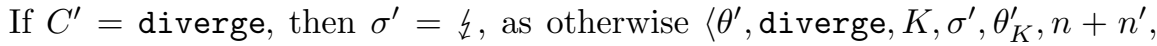
$\left.w^{\prime}\right\rangle$ would reduce by (diverge). However, $\left\langle\theta^{\prime}\right.$, diverge, $\left.K, \downarrow, \theta_{K}^{\prime}, n+n^{\prime}, w^{\prime}\right\rangle$ is not derivable from any initial configuration other than itself. Hence, $n^{\prime}=0$ and $k=0$ and $\sigma=\xi$. Since no configuration with state $\downarrow$ reduces, we have $\langle\theta$, while $\left.{ }^{m}(\phi)\{C\},[], \downarrow, \theta_{K}, n, w\right\rangle \nvdash$, as required. 
The above results lead to the following properties of semantic functions:

Corollary 11. If $n \geq k$, then $\mathbf{O}_{\text {while }^{n}(\phi)\{C\}}^{\sigma}(\theta) \geq \mathbf{O}_{\mathrm{while}^{k}(\phi)\{C\}}^{\sigma}(\theta)$ (w.r.t. flat CPO with bottom $\uparrow)$.

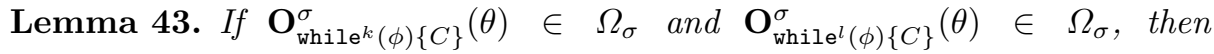

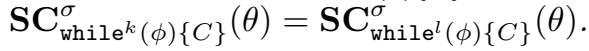

Proof. Assume w.l.o.g. that $l \geq k$. Then the result follows directly from Lemma 41

Proofs of Propositions 3 and 4 Having shown the above properties of whileloop approximations, we are now ready to prove Propositions 3 and 4

Restatement of Proposition 4 If $n \geq k$, then $\hat{f}\left(\mathbf{O}_{\text {while }^{n}(\phi)\{C\}}^{\sigma}(\theta)\right)$

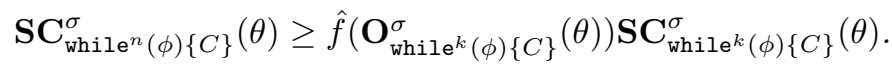

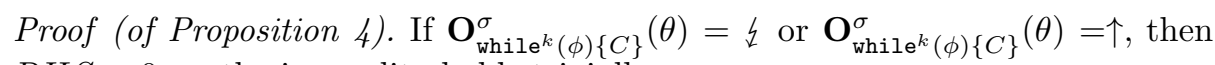
$R H S=0$, so the inequality holds trivially.

If $\mathbf{O}_{\text {while }^{k}(\phi)\{C\}}^{\sigma}(\theta) \in \Omega_{\sigma}$, then by Corollary 11] $\mathbf{O}_{\text {while }^{n}(\phi)\{C\}}^{\sigma}(\theta)=$ $\mathbf{O}_{\text {while }}^{\sigma}(\phi)\{C\}(\theta)$ and by Lemma 43. $\mathbf{S C}_{\text {while }}^{\sigma}(\phi)\{C\}(\theta)=$ $\mathbf{S C}_{\text {while }^{k}(\phi)\{C\}}^{\sigma}(\theta)$. Hence, $\quad f\left(\mathbf{O}_{\text {while }^{n}(\phi)\{C\}}^{\sigma}(\theta)\right) \mathbf{S C}_{\text {while }^{n}(\phi)\{C\}}^{\sigma}(\theta)=$ $f\left(\mathbf{O}_{\text {while }^{k}(\phi)\{C\}}^{\sigma}(\theta)\right) \mathbf{S C}_{\text {while }^{k}(\phi)\{C\}}^{\sigma}(\theta)$.

Restatement of Proposition $3 \hat{f}\left(\mathbf{O}_{\text {while }(\phi)\{C\}}^{\sigma}(\theta)\right) \mathbf{S C}_{\text {while }(\phi)\{C\}}^{\sigma}(\theta)=$ $\sup _{n} \hat{f}\left(\mathbf{O}_{\text {while }^{n}(\phi)\{C\}}^{\sigma}(\theta)\right) \mathbf{S C}_{\text {while }^{n}(\phi)\{C\}}^{\sigma}(\theta)$

Proof (of Proposition [3). If $\mathbf{O}_{\text {while }(\phi)\{C\}}^{\sigma}(\theta) \notin \Omega_{\sigma}$, then $L H S=0$. If

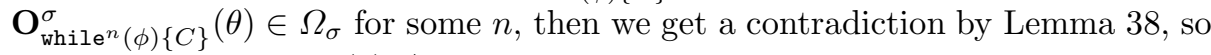
we have $\mathbf{O}_{\text {while }^{n}(\phi)\{C\}}^{\sigma}(\theta) \notin \Omega_{\sigma}$, which implies $R H S=0$.

Now, assume that $\mathbf{O}_{\text {while }(\phi)\{C\}}^{\sigma}(\theta) \in \Omega_{\sigma}$. Then by Lemma 35, there ex-

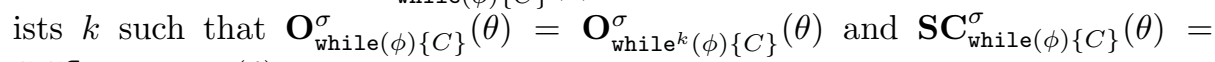
$\mathbf{S C}_{\text {while }}^{\sigma}(\phi)\{C\}(\theta)$.

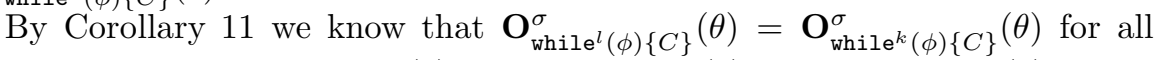

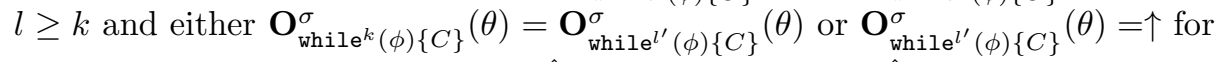

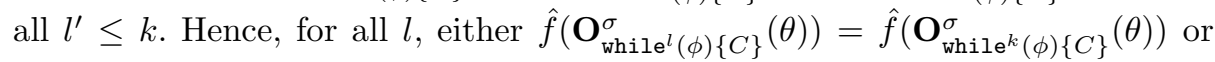

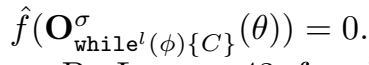

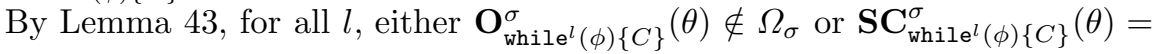

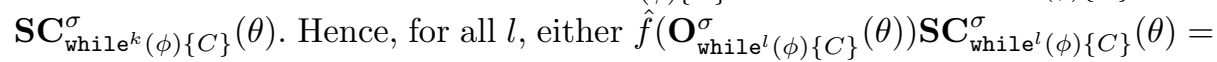

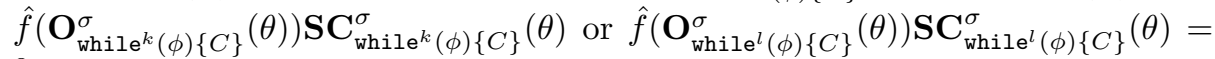
0 .

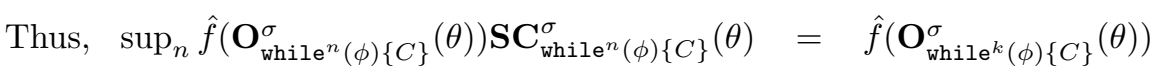
$\mathbf{S C}_{\text {while }}^{\sigma}(\phi)\{C\}(\theta)$, and so $\hat{f}\left(\mathbf{O}_{\text {while }(\phi)\{C\}}^{\sigma}(\theta)\right) \mathbf{S C}_{\text {while }(\phi)\{C\}}^{\sigma}(\theta)=$ $\sup _{n} \hat{f}\left(\mathbf{O}_{\text {while }^{n}(\phi)\{C\}}^{\sigma}(\theta)\right) \mathbf{S C}_{\text {while }^{n}(\phi)\{C\}}^{\sigma}(\theta)$, as required. 
Proofs of Propositions 5 and 6 Finally, we prove Propositions 5 and 6 , which are required by Theorem 2. One final additional result needed for these proofs

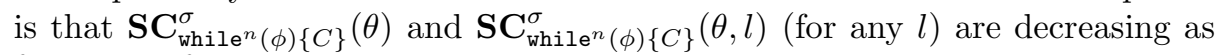
functions of $n$.

Lemma 44. If $n \geq k$, then $\mathbf{S C}_{\text {while }^{n}(\phi)\{C\}}^{\sigma}(\theta) \leq \mathbf{S C}_{\text {while }^{k}(\phi)\{C\}}^{\sigma}(\theta)$.

Proof. If $\mathbf{O}_{\text {while }}^{\sigma}(\phi)\{C\}(\theta)=\downarrow$, then $\mathbf{O}_{\text {while }^{n}(\phi)\{C\}}^{\sigma}(\theta)=\downarrow$ by Corollary 11, Hence, $\mathbf{S C}_{\text {while }^{k}(\phi)\{C\}}^{\sigma}(\theta)=\mathbf{S C}_{\text {while }}^{\sigma}(\phi)\{C\}(\theta)=0$.

Now, suppose that $\mathbf{O}_{\text {while }}^{\sigma}(\phi)\{C\}(\theta) \neq$. If there exists $l$ such that $\left\langle\theta\right.$, while $\left.{ }^{k}(\phi)\{C\},[], \sigma, \theta_{K}, 0,1\right\rangle \quad \vdash_{\min }^{*}\left\langle\theta^{\prime}\right.$, diverge, $\left.K, \tau, \theta_{K}^{\prime}, l, w\right\rangle$, then by Lemma 45] $\left\langle\theta\right.$, while $\left.{ }^{n}(\phi)\{C\},[], \sigma, \theta_{K}, 0,1\right\rangle \vdash^{*}\left\langle\theta^{\prime}, C, K^{\prime}, \tau, \theta_{K}^{\prime}, l, w\right\rangle$ and $\left\langle\theta^{\prime}\right.$, diverge, $\left.K, \tau, \theta_{K}^{\prime}, l, w\right\rangle \unlhd\left\langle\theta^{\prime}, C, K^{\prime}, \tau, \theta_{K}^{\prime}, l, w\right\rangle$. Since $\left\langle\theta^{\prime}\right.$, diverge, $K, \tau, \theta_{K}^{\prime}$, $m, w\rangle \vdash\left\langle\theta^{\prime}\right.$, diverge, $\left.K, \tau, \theta_{K}^{\prime}, m+1, w\right\rangle$, for all $l^{\prime} \geq l$, we have

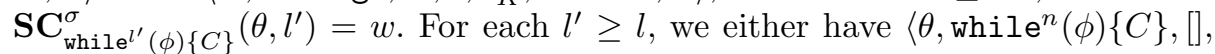
$\left.\sigma, \theta_{K}, 0,1\right\rangle \vdash^{*}\left\langle\theta^{\prime}, C, K^{\prime}, \tau, \theta_{K}^{\prime}, l, w\right\rangle \vdash^{*}\left\langle\theta^{\prime \prime}, C^{\prime}, K^{\prime \prime}, \tau^{\prime}, \theta_{K}^{\prime \prime}, l^{\prime}, w^{\prime}\right\rangle$, where $w^{\prime} \leq w^{\prime}$ by Lemma 7 and so $\mathbf{S C}_{\text {while }}^{\sigma}(\phi)\{C\}\left(\theta, l^{\prime}\right)=w^{\prime}$ or while ${ }^{n}(\phi)\{C\}$ does not re-

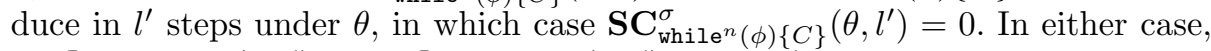

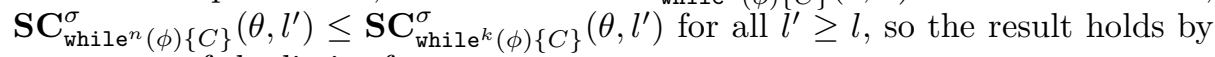
a property of the limit of a sequence.

If there exists no $l$ such that $\left\langle\theta\right.$, while ${ }^{k}(\phi)\{C\}$, [], $\left.\sigma, \theta_{K}, 0,1\right\rangle \vdash_{\text {min }}^{*}\left\langle\theta^{\prime}\right.$, diverge, $\left.K, \tau, \theta_{K}^{\prime}, l, w\right\rangle$, then for all $l$, we have $\left\langle\theta, \operatorname{while}^{k}(\phi)\{C\},[], \sigma, \theta_{K}\right.$, $0,1\rangle \quad \vdash^{*}\left\langle\theta^{\prime}, C, K, \tau, \theta_{K}^{\prime}, l, w\right\rangle$, where $C \neq$ diverge. By Corollary 9, $\left\langle\theta\right.$, while $\left.{ }^{n}(\phi)\{C\},[], \sigma, \theta_{K}, 0,1\right\rangle \vdash^{*}\left\langle\theta^{\prime}, C^{\prime}, K^{\prime}, \tau, \theta_{K}^{\prime}, l, w\right\rangle$ for some $C^{\prime}, K^{\prime}$, and so $\mathbf{S C}_{\text {while }}^{\sigma}(\phi)\{C\}(\theta, l)=\mathbf{S C}_{\text {while }}^{\sigma}(\phi)\{C\}(\theta, l)$ for all $l$, which implies

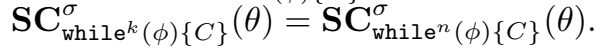

Lemma 45. If $\left\langle\theta, C, K, \sigma, \theta_{K}, m, w\right\rangle \unlhd\left\langle\theta, C^{\prime}, K^{\prime}, \sigma, \theta_{K}, m, w\right\rangle$ and $\left\langle\theta, C, K, \sigma, \theta_{K}\right.$, $m, w\rangle \vdash_{\text {min }}^{*}\left\langle\theta^{\prime \prime}\right.$, diverge, $\left.K^{\prime \prime}, \sigma^{\prime \prime}, \theta_{K}^{\prime \prime}, m+n^{\prime}, w^{\prime \prime}\right\rangle$ then

$\left\langle\theta, C^{\prime}, K^{\prime}, \sigma, \theta_{K}, m, w\right\rangle \vdash^{*}\left\langle\theta^{\prime \prime}, C^{\prime \prime \prime}, K^{\prime \prime \prime}, \sigma^{\prime \prime}, \theta_{K}^{\prime \prime}, m+n^{\prime}, w^{\prime \prime}\right\rangle$ and $\left\langle\theta^{\prime \prime}\right.$, diverge, $\left.K^{\prime \prime}, \sigma^{\prime \prime}, \theta_{K}^{\prime \prime}, m+n^{\prime}, w^{\prime \prime}\right\rangle \unlhd\left\langle\theta^{\prime \prime}, C^{\prime \prime \prime}, K^{\prime \prime \prime}, \sigma^{\prime \prime}, \theta_{K}^{\prime \prime}, m+n^{\prime}, w^{\prime \prime}\right\rangle$

Proof. Follows from Corollary 9 and Lemma 37.

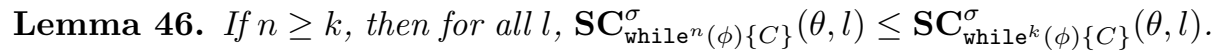

Proof. If $\left\langle\theta\right.$, while ${ }^{k}(\phi)\{C\}$, [], $\left.\sigma, \theta_{K}, 0,1\right\rangle \vdash^{*}\left\langle\theta^{\prime}, C^{\prime}, K, \sigma^{\prime}, \theta_{K}^{\prime}, l^{\prime}, w\right\rangle \nvdash$ for some $l^{\prime}<l$, then

$\left\langle\theta\right.$, while $\left.{ }^{n}(\phi)\{C\},[], \sigma, \theta_{K}, 0,1\right\rangle \vdash^{*}\left\langle\theta^{\prime}, C^{\prime \prime}, K^{\prime}, \sigma^{\prime}, \theta_{K}^{\prime}, l^{\prime}, w\right\rangle \nvdash$ by Lemma 42, and

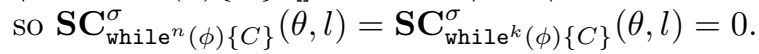

If $\left\langle\theta\right.$, while ${ }^{k}(\phi)\{C\}$, [], $\left.\sigma, \theta_{K}, 0,1\right\rangle \vdash^{*}\left\langle\theta^{\prime}\right.$, diverge, $\left.K, \sigma^{\prime}, \theta_{K}^{\prime}, l, w\right\rangle$, then $\mathbf{S C}_{\text {while }}^{\sigma}(\phi)\{C\}(\theta, l)=w$ and there must exist a $l^{\prime} \leq l$ such that $\langle\theta$, while $^{k}(\phi)\{C\}$, [], $\left.\sigma, \theta_{K}, 0,1\right\rangle \vdash_{\text {min }}^{*}\left\langle\theta^{\prime}\right.$, diverge, $\left.K, \sigma^{\prime}, \theta_{K}^{\prime}, l^{\prime}, w\right\rangle$. Moreover, by Lemma 45. $\left\langle\theta\right.$, while $\left.{ }^{n}(\phi)\{C\},[], \sigma, \theta_{K}, 0,1\right\rangle \vdash^{*}\left\langle\theta^{\prime}, C^{\prime \prime}, K^{\prime}, \tau, \theta_{K}^{\prime}, l^{\prime}, w\right\rangle$ and $\left\langle\theta^{\prime}\right.$, diverge, $\left.K, \tau, \theta_{K}^{\prime}, l^{\prime}, w\right\rangle \unlhd\left\langle\theta^{\prime}, C^{\prime \prime}, K^{\prime}, \tau, \theta_{K}^{\prime}, l^{\prime}, w\right\rangle$. If we have $\left\langle\theta^{\prime}, C^{\prime \prime}, K^{\prime}, \tau, \theta_{K}^{\prime}, l^{\prime}\right.$, $w\rangle \vdash^{*}\left\langle\theta^{\prime \prime}, C^{\prime \prime \prime}, K^{\prime \prime}, \tau, \theta_{K}^{\prime \prime}, l, w^{\prime}\right\rangle$, then $\mathbf{S C}_{\text {while }}^{\sigma}(\phi)\{C\}(\theta, l)=w^{\prime} \leq w$ by Lemma 7 . 


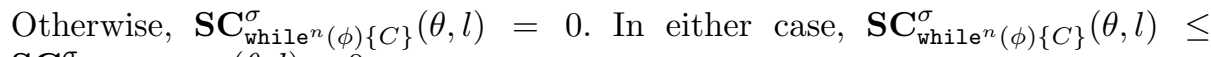
$\mathbf{S C}_{\text {while }^{k}(\phi)\{C\}}^{\sigma}(\theta, l)=0$.

If $\left\langle\theta\right.$, while ${ }^{k}(\phi)\{C\}$, [], $\left.\sigma, \theta_{K}, 0,1\right\rangle \quad \vdash^{*}\left\langle\theta^{\prime}, C^{\prime}, K, \sigma^{\prime}, \theta_{K}^{\prime}, l, w\right\rangle$ and $C^{\prime} \neq$ diverge, then by Corollary [9, $\left\langle\theta\right.$, while $\left.{ }^{n}(\phi)\{C\},[], \sigma, \theta_{K}, 0,1\right\rangle \vdash\left\langle\theta^{\prime}\right.$, $\left.C^{\prime \prime}, K^{\prime}, \tau, \theta_{K}^{\prime}, l, w\right\rangle$ and $\left\langle\theta^{\prime}, C^{\prime}, K, \tau, \theta_{K}^{\prime}, l, w\right\rangle \unlhd\left\langle\theta^{\prime}, C^{\prime \prime}, K^{\prime}, \tau, \theta_{K}^{\prime}, l, w\right\rangle$. Thus,

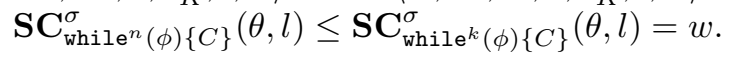

Restatement of Proposition 5 For all $f \leq 1$,

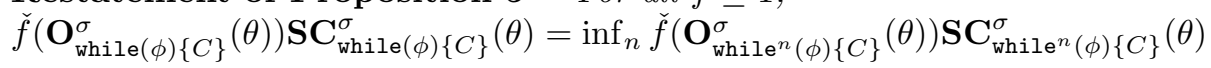

Proof (of Proposition 5). If $\mathbf{O}_{\text {while }(\phi)\{C\}}^{\sigma}(\theta) \in \Omega_{\sigma}$, then by Lemma 35, there

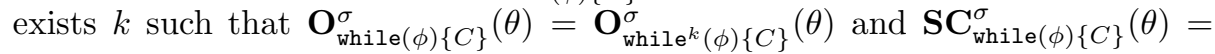
$\mathbf{S C}_{\text {while }}^{\sigma}(\phi)\{C\}(\theta)$. By similar reasoning as in the proof of Proposition 3 , for all

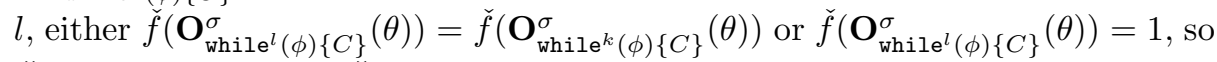
$\check{f}\left(\mathbf{O}_{\text {while }^{l}(\phi)\{C\}}^{\sigma}(\theta)\right) \geq \check{f}\left(\mathbf{O}_{\text {while }^{k}(\phi)\{C\}}^{\sigma}(\theta)\right)$ for all $l$.

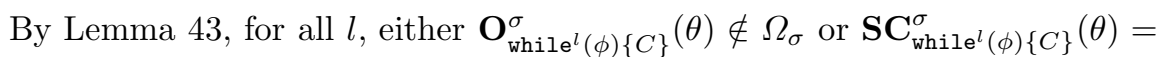

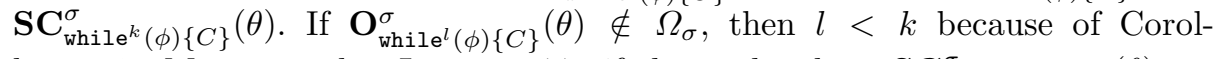
lary [1] Moreover, by Lemma [44, if $l<k$, then $\operatorname{SC}_{\text {while }}^{\sigma}(\phi)\{C\}(\theta) \leq$

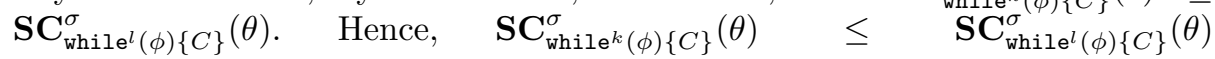
for all $l$. This implies $\inf _{n} \check{f}\left(\mathbf{O}_{\text {while }}^{\sigma}(\phi)\{C\}(\theta)\right) \mathbf{S C}_{\text {while }}^{\sigma}(\phi)\{C\}(\theta)=$

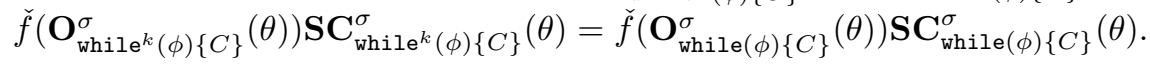

If $\mathbf{O}_{\text {while }(\phi)\{C\}}^{\sigma}(\theta)=$ k, then by Lemma 36. $\mathbf{O}_{\text {while }}^{\sigma}(\phi)\{C\}(\theta)=$

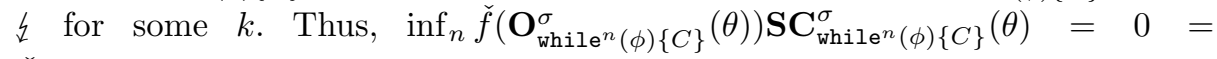
$\check{f}\left(\mathbf{O}_{\text {while }(\phi)\{C\}}^{\sigma}(\theta)\right) \mathbf{S C}_{\text {while }(\phi)\{C\}}^{\sigma}(\theta)$.

If $\mathbf{O}_{\text {while }(\phi)\{C\}}^{\sigma}(\theta)=\uparrow$, then $\check{f}\left(\mathbf{O}_{\text {while }(\phi)\{C\}}^{\sigma}(\theta)\right)=1$. By Lemma 10, $\mathbf{O}_{\text {while }}^{\sigma}(\phi)\{C\}(\theta)=\uparrow$ for all $k$. Since $\check{f}(\uparrow)=1$, we only need to show that $\mathbf{S C}_{\text {while }(\phi)\{C\}}^{\sigma}(\theta)=\inf _{n} \mathbf{S C}_{\text {while }^{n}(\phi)\{C\}}^{\sigma}(\theta)$.

First, observe that from Corollary 8, it follows that for all $l$, for all $k \geq l, \mathbf{S C}_{\text {while }(\phi)\{C\}}^{\sigma}(\theta, l)=\mathbf{S C}_{\text {while }^{k}(\phi)\{C\}}^{\sigma}(\theta, l)$. Thus, for such fixed $l$,

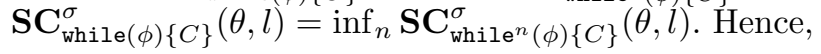

$$
\begin{aligned}
\mathbf{S C}_{\text {while }(\phi)\{C\}}^{\sigma}(\theta) & =\inf _{l} \mathbf{S C}_{\text {while }(\phi)\{C\}}^{\sigma}(\theta, l) \\
& =\inf _{l} \inf _{n} \mathbf{S C}_{\text {while }^{n}(\phi)\{C\}}^{\sigma}(\theta, l) \\
& =\inf _{n} \inf _{l} \mathbf{S C}_{\text {while }^{n}(\phi)\{C\}}^{\sigma}(\theta, l) \\
& =\inf _{n} \mathbf{S C}_{\text {while }^{n}(\phi)\{C\}}^{\sigma}(\theta)
\end{aligned}
$$

In the equality $\inf _{l} \inf _{n} \mathbf{S C}_{\text {while }^{n}(\phi)\{C\}}^{\sigma}(\theta, l)=\inf _{n} \inf _{l} \mathbf{S C}_{\text {while }}^{\sigma}(\phi)\{C\}(\theta, l)$, we used the fact that $\inf _{l} \inf _{n} \mathbf{S C}_{\text {while }^{n}(\phi)\{C\}}^{\sigma}(\theta, l)=$ 


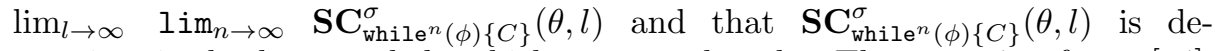
creasing in both $n$ and $l$, which means that by Theorem 4.2 from [14], $\lim _{l \rightarrow \infty} \lim _{n \rightarrow \infty} \mathbf{S C}_{\text {while }^{n}(\phi)\{C\}}^{\sigma}(\theta, l)=\lim _{n \rightarrow \infty} \lim _{l \rightarrow \infty} \mathbf{S C}_{\text {while }^{n}(\phi)\{C\}}^{\sigma}(\theta, l)$.

Below, we write $\left\langle\theta, C, K, \sigma, \theta_{K}, n, w\right\rangle \vdash_{\min }^{*}\left\langle\theta^{\prime}\right.$, diverge, $\left.K^{\prime}, \sigma^{\prime}, \theta_{K}^{\prime}, n+n^{\prime}, w^{\prime}\right\rangle$ if $\left\langle\theta, C, K, \sigma, \theta_{K}, n, w\right\rangle \vdash^{*}\left\langle\theta^{\prime}\right.$, diverge, $\left.K^{\prime}, \sigma^{\prime}, \theta_{K}^{\prime}, n+n^{\prime}, w^{\prime}\right\rangle$ and there is no $n^{\prime \prime}<n^{\prime}$ such that $\left\langle\theta, C, K, \sigma, \theta_{K}, n, w\right\rangle \vdash^{*}\left\langle\theta^{\prime \prime}\right.$, diverge, $\left.K^{\prime \prime}, \sigma^{\prime \prime}, \theta_{K}^{\prime \prime}, n+n^{\prime \prime}, w^{\prime \prime}\right\rangle$ (or, equivalently, $\left\langle\theta, C, K, \sigma, \theta_{K}, n, w\right\rangle \vdash^{*}\left\langle\theta^{\prime}\right.$, diverge, $\left.K^{\prime}, \sigma^{\prime}, \theta_{K}^{\prime}, n+n^{\prime}, w^{\prime}\right\rangle$ was derived without (diverge)).

Restatement of Proposition 6 If $n \geq k$ and $f \leq 1$, then $\check{f}\left(\mathbf{O}_{\text {while }^{n}(\phi)\{C\}}^{\sigma}(\theta)\right) \mathbf{S C}_{\text {while }^{n}(\phi)\{C\}}^{\sigma}(\theta) \leq \check{f}\left(\mathbf{O}_{\text {while }^{k}(\phi)\{C\}}^{\sigma}(\theta)\right) \mathbf{S C}_{\text {while }^{k}(\phi)\{C\}}^{\sigma}(\theta)$.

Proof (of Proposition [6). By Corollary 11, $\mathbf{O}_{\text {while }}^{\sigma}(\phi)\{C\}(\theta) \geq \mathbf{O}_{\text {while }^{k}(\phi)\{C\}}^{\sigma}(\theta)$. Since $\check{f}$ is antitone (we have $\check{f}(\tau) \leq \check{f}(\uparrow)=1$ for all $\tau \geq \uparrow$ ), this implies $\check{f}\left(\mathbf{O}_{\text {while }}^{\sigma}(\phi)\{C\}(\theta)\right) \leq \breve{f}\left(\mathbf{O}_{\text {while }^{k}(\phi)\{C\}}^{\sigma}(\theta)\right)$. By Lemma 44, $\mathbf{S C}_{\text {while }^{n}(\phi)\{C\}}^{\sigma}(\theta) \leq$

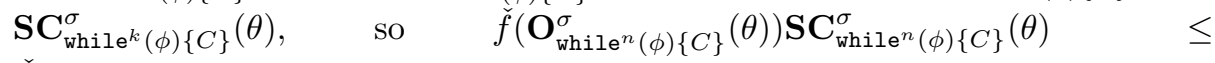
$\check{f}\left(\mathbf{O}_{\text {while }^{k}(\phi)\{C\}}^{\sigma}(\theta)\right) \mathbf{S C}_{\text {while }^{k}(\phi)\{C\}}^{\sigma}(\theta)$, as required.

\section{E Proofs of Theorems 1 and 2}

Restatement of Theorem 11 For all measurable functions $f: \Omega_{\sigma} \rightarrow \overline{\mathbb{R}}_{+}$, $P L$ programs $C$ and initial states $\sigma \in \Omega_{\sigma}$ :

$$
\mathrm{wp} \llbracket C \rrbracket(f)(\sigma)=\int f(\tau) \llbracket C \rrbracket_{\sigma}(d \tau) .
$$

Proof. By Lemma 10, it suffices to prove that for all $f$ :

$$
\int \hat{f}\left(\mathbf{O}_{C}^{\sigma}(\theta)\right) \cdot \mathbf{S C}_{C}^{\sigma}(\theta) \mu_{\mathbb{S}}(d \theta)=\operatorname{wp} \llbracket C \rrbracket(f)(\sigma) .
$$

This can be proven by induction on the structure of $C$. We refrain from treating all cases but restrict ourselves to some interesting cases:

- Case $C=x: \approx U$.

$$
\begin{aligned}
& \int \hat{f}\left(\mathbf{O}_{x: \approx U}^{\sigma}(\theta)\right) \cdot \mathbf{S C}_{x: \approx U}^{\sigma}(\theta) \mu_{\mathbb{S}}(d \theta) \\
= & \int f\left(\sigma\left[x \mapsto \pi_{U}\left(\pi_{L}(\theta)\right)\right]\right) \mu_{\mathbb{S}}(d \theta) \\
\text { (property entropy) }= & \int_{[0,1]} f(\sigma[x \mapsto v]) \mu_{L}(d v) \\
\text { (definition up) }= & \operatorname{wp} \llbracket x: \approx U \rrbracket(f)(\sigma) .
\end{aligned}
$$


- Case $C=C_{1} ; C_{2}$ with $C_{1} \neq C_{1}^{\prime} ; C_{2}^{\prime}$.

$$
\begin{aligned}
& \int \hat{f}\left(\mathbf{O}_{C_{1} ; C_{2}}^{\sigma}(\theta)\right) \cdot \mathbf{S C}_{C_{1} ; C_{2}}^{\sigma}(\theta) \mu_{\mathbb{S}}(d \theta) \\
& \text { (Proposition凹 }=\int \hat{f}\left(\mathbf{O}_{C_{2}}^{\tau}\left(\pi_{R}(\theta)\right)\right) \cdot \mathbf{S C}_{C_{2}}^{\tau}\left(\pi_{R}(\theta)\right) \cdot \mathbf{S C}_{C_{1}}^{\sigma}\left(\pi_{L}(\theta)\right) \mu_{\mathbb{S}}(d \theta) \\
& \text { (property entropy) }=\int \underbrace{\int \hat{f}\left(\mathbf{O}_{C_{2}}^{\rho}\left(\theta_{R}\right)\right) \cdot \mathbf{S C}_{C_{2}}^{\rho}\left(\theta_{R}\right) \mu_{\mathbb{S}}\left(d \theta_{R}\right)}_{=g(\rho)} \cdot \mathbf{S C}_{C_{1}}^{\sigma}\left(\theta_{L}\right) \mu_{\mathbb{S}}\left(d \theta_{L}\right)
\end{aligned}
$$

where $\tau=\mathbf{O}_{C_{1}}^{\sigma}\left(\pi_{L}(\theta)\right)$ and $\rho=\mathbf{O}_{C_{1}}^{\sigma}\left(\theta_{L}\right)$. We have:

$$
\begin{aligned}
& \int \hat{g}\left(\mathbf{O}_{C_{1}}^{\sigma}\left(\theta_{L}\right)\right) \cdot \mathbf{S C}_{C_{1}}^{\sigma}\left(\theta_{L}\right) \mu_{\mathbb{S}}\left(d \theta_{L}\right) \\
\text { (induction hypothesis) } & =\operatorname{wp} \llbracket C_{1} \rrbracket(g)(\sigma) \\
= & \operatorname{wp} \llbracket C_{1} \rrbracket\left(\lambda \tau \cdot \int \hat{f}\left(\mathbf{O}_{C_{2}}^{\tau}\left(\theta_{R}\right)\right) \cdot \mathbf{S C}_{C_{2}}^{\tau}\left(\theta_{R}\right) \mu_{\mathbb{S}}\left(d \theta_{R}\right)\right)(\sigma) \\
\text { (induction hypothesis) } & =\operatorname{wp} \llbracket C_{1} \rrbracket\left(\lambda \tau \cdot \operatorname{wp} \llbracket C_{2} \rrbracket(f)(\tau)\right)(\sigma) \\
= & w p \llbracket C_{1} \rrbracket\left(\operatorname{wp} \llbracket C_{2} \rrbracket(f)\right)(\sigma) \\
\text { (definition wp) }= & \operatorname{wp} \llbracket C_{1} ; C_{2} \rrbracket(f)(\sigma)
\end{aligned}
$$

- Case $C=\operatorname{score}(E)$. By inspecting the reduction rules, it follows:

$$
\mathbf{O}_{\text {score }(E)}^{\sigma}(\theta)= \begin{cases}\sigma & \text { if } \sigma(E) \in(0,1] \\ z & \text { otherwise }\end{cases}
$$

which implies $\hat{f}\left(\mathbf{O}_{\operatorname{score}(E)}^{\sigma}(\theta)\right)=[\sigma(E) \in(0,1]] \cdot \hat{f}(\sigma)$ and

$$
\mathbf{S C}_{\text {score }(E)}^{\sigma}(\theta)=\left\{\begin{array}{ll}
\sigma(E) & \text { if } \sigma(E) \in(0,1] \\
0 & \text { otherwise }
\end{array}=[\sigma(E) \in(0,1]] \cdot \sigma(E) .\right.
$$

Thus, we have:

$$
\begin{aligned}
& \int \hat{f}\left(\mathbf{O}_{\mathrm{score}(E)}^{\sigma}(\theta)\right) \cdot \mathbf{S C}_{\mathrm{score}(E)}^{\sigma}(\theta) \mu_{\mathbb{S}}(d \theta) \\
= & \int[\sigma(E) \in(0,1]] \cdot \hat{f}(\sigma) \cdot \sigma(E) \mu_{\mathbb{S}}(d \theta) \\
= & {[\sigma(E) \in(0,1]] \cdot \hat{f}(\sigma) \cdot \sigma(E) } \\
\left(\sigma \in \Omega_{\sigma \text { by assumption })}=\right. & {[\sigma(E) \in(0,1]] \cdot f(\sigma) \cdot \sigma(E) } \\
= & \operatorname{wp} \llbracket \operatorname{score}(E) \rrbracket(f)(\sigma) .
\end{aligned}
$$


- Case $C=$ while $(\phi)\left\{C^{\prime}\right\}$. Let $C^{n}=$ while ${ }^{n}(\phi)\left\{C^{\prime}\right\}$. We derive:

$$
\begin{aligned}
& \int \hat{f}\left(\mathbf{O}_{C}^{\sigma}(\theta)\right) \cdot \mathbf{S C}_{C}^{\sigma}(\theta) \mu_{\mathbb{S}}(d \theta) \\
& { }_{\text {(Proposition [3] }}=\int \sup _{n} \hat{f}\left(\mathbf{O}_{C^{n}}^{\sigma}(\theta)\right) \cdot \mathbf{S C}_{C^{n}}^{\sigma}(\theta) \mu_{\mathbb{S}}(d \theta) \\
& \text { (Beppo Levi's Theorem) }=\sup _{n} \int \hat{f}\left(\mathbf{O}_{C^{n}}^{\sigma}(\theta)\right) \cdot \mathbf{S C}_{C^{n}}^{\sigma}(\theta) \mu_{\mathbb{S}}(d \theta) \\
& \left.{ }^{*}\right)=\sup _{n} \operatorname{wp}_{\left\langle\phi, C^{\prime}\right\rangle} \Phi_{f}^{n}(0)(\sigma) \\
& { }_{(\text {Kleene's Fixpoint Theorem) }}=\mathrm{wp} \llbracket \text { while }(\phi)\left\{C^{\prime}\right\} \rrbracket(f)(\sigma) .
\end{aligned}
$$

When applying the Beppo Levi's Theorem, we used the fact that the sequence $\hat{f}\left(\mathbf{O}_{C^{n}}^{\sigma}(\theta)\right) \cdot \mathbf{S C}_{C^{n}}^{\sigma}(\theta)$ is monotonic in $n$ (Proposition 4). In order to show that the proof step $(*)$ is correct, we need to show:

$$
\int \hat{f}\left(\mathbf{O}_{C^{n}}^{\sigma}(\theta)\right) \cdot \mathbf{S C}_{C^{n}}^{\sigma}(\theta) \mu_{\mathbb{S}}(d \theta)={ }_{\left\langle\phi, C^{\prime}\right\rangle}^{\mathrm{wp}} \Phi_{f}^{n}(0)(\sigma) \text { for all } n .
$$

We prove this statement by induction on $n$, using Proposition 2

- Base case: $n=0$ :

$$
\int \underbrace{\hat{f}\left(\mathbf{O}_{\text {diverge }}^{\sigma}(\theta)\right)}_{=0} \cdot \underbrace{\mathbf{S C}_{\text {diverge }}^{\sigma}(\theta) \mu_{\mathbb{S}}(d \theta)}_{=1}=0={ }_{\left\langle\phi, C^{\prime}\right\rangle}^{\mathrm{wp}} \Phi_{f}^{0}(0)(\sigma)
$$

- Induction step: we distinguish $\sigma(\phi)=$ true and $\sigma(\phi)=$ false. For the latter case we have:

$$
\int \hat{f}(\sigma) \cdot 1 \mu_{\mathbb{S}}(d \theta)=f(\sigma)
$$

For the case $\sigma(\phi)=$ true we derive:

$$
\begin{aligned}
& \int \hat{f}\left(\mathbf{O}_{C^{n+1}}^{\sigma}(\theta)\right) \cdot \mathbf{S C}_{C^{n+1}}^{\sigma}(\theta) \mu_{\mathbb{S}}(d \theta) \\
& =\int \hat{f}\left(\mathbf{O}_{C^{\prime} ; C^{n}}^{\sigma}(\theta)\right) \cdot \mathbf{S C}_{C^{\prime} ; C^{n}}^{\sigma}(\theta) \mu_{\mathbb{S}}(d \theta) \\
& \text { (Prop. [■ }=\int \hat{f}\left(\mathbf{O}_{C^{n}}^{\tau}\left(\pi_{R}(\psi(\theta))\right)\right) \cdot \mathbf{S C}_{C^{\prime}}^{\sigma}\left(\pi_{L}(\psi(\theta))\right) \cdot \mathbf{S C}_{C^{n}}^{\tau}\left(\pi_{R}(\psi(\theta))\right) \mu_{\mathbb{S}}(d \theta) \\
& \text { (Prop' [32] }=\int \hat{f}\left(\mathbf{O}_{C^{n}}^{\rho}\left(\pi_{R}(\theta)\right)\right) \cdot \mathbf{S C}_{C^{\prime}}^{\sigma}\left(\pi_{L}(\theta)\right) \cdot \mathbf{S C}_{C^{n}}^{\rho}\left(\pi_{R}(\theta)\right) \mu_{\mathbb{S}}(d \theta) \\
& { }_{\text {(entropy) }}=\iint \hat{f}\left(\mathbf{O}_{C^{n}}^{\rho}\left(\theta_{R}\right)\right) \cdot \mathbf{S C}_{C^{n}}^{\rho}\left(\theta_{R}\right) \mu_{\mathbb{S}}\left(d \theta_{R}\right) \cdot \mathbf{S C}_{C^{\prime}}^{\sigma}\left(\theta_{L}\right) \mu_{\mathbb{S}}\left(d \theta_{L}\right)
\end{aligned}
$$

where $\tau=\mathbf{O}_{C^{\prime}}^{\sigma}\left(\pi_{L}(\psi(\theta))\right)$ and $\rho=\mathbf{O}_{C^{\prime}}^{\sigma}\left(\pi_{L}(\theta)\right)$. 
Now let $p(\tau)=\int \hat{f}\left(\mathbf{O}_{C^{n}}^{\tau}\left(\theta_{R}\right)\right) \cdot \mathbf{S C}_{C^{n}}^{\tau}\left(\theta_{R}\right) \mu_{\mathbb{S}}\left(d \theta_{R}\right)$ for $\tau \in \Omega_{\sigma}$. Then:

$$
\begin{aligned}
& \int \hat{p}\left(\mathbf{O}_{C}^{\sigma}\left(\theta_{L}\right)\right) \cdot \mathbf{S C}_{C}^{\sigma}\left(\theta_{L}\right) \mu_{\mathbb{S}}\left(d \theta_{L}\right) \\
& \text { (outer } \mathrm{IH})=\operatorname{wp} \llbracket C \rrbracket(p)(\sigma) \\
& =\operatorname{wp} \llbracket C \rrbracket\left(\lambda \tau \cdot \int \hat{f}\left(\mathbf{O}_{C^{n}}^{\tau}\left(\theta_{R}\right)\right) \cdot \mathbf{S C}_{C^{n}}^{\tau}\left(\theta_{R}\right) \mu_{\mathbb{S}}\left(d \theta_{R}\right)\right)(\sigma) \\
& { }_{\text {(inner IH) }}=\operatorname{wp} \llbracket C \rrbracket\left(\lambda \tau \cdot{ }_{\left\langle\phi, C^{\prime}\right\rangle}^{\mathrm{wp}} \Phi_{f}^{n}(0)(\tau)\right)(\sigma) \\
& =\operatorname{wp} \llbracket C \rrbracket\left({ }_{\left\langle\phi, C^{\prime}\right\rangle} \Phi_{f}^{n}(0)\right)(\sigma) \\
& \text { (definition }{ }_{\langle\phi, C\rangle}^{\left.\mathrm{wp}_{f}\right)}={ }_{\left\langle\phi, C^{\prime}\right\rangle} \Phi_{f}^{n+1}(0)(\sigma) .
\end{aligned}
$$

Hence, the equality $(*)$ is correct, which finishes the proof.

The second main theorem of this paper states that the weakest liberal preexpectation of a non-negative function $f$ bounded by 1 is equivalent to the expected value of $f$ with respect to the distribution defined by the operational semantics plus the probability of divergence weighted by scores.

Restatement of Theorem [2 For every measurable non-negative function $f: \Omega_{\sigma} \rightarrow \overline{\mathbb{R}}_{+}$with $f(\sigma) \leq 1$ for all states $\sigma$, PL program $C$ and initial state $\sigma \in \Omega_{\sigma}$ :

$$
\mathrm{wlp} \llbracket C \rrbracket(f)(\sigma)=\left.\int f(\tau) \cdot \llbracket C \rrbracket_{\sigma}\right|_{\Omega_{\sigma}}(d \tau)+\underbrace{\int\left[\mathbf{O}_{C}^{\sigma}(\theta)=\uparrow\right] \cdot \mathbf{S C}_{C}^{\sigma}(\theta) \mu_{\mathbb{S}}(d \theta)}_{\text {probability of divergence multiplied by the score }} .
$$

Proof. By induction on the structure of $C$. The proof is essentially the same as the proof of Theorem 1, except that in the case of while-loops, we use Proposition 5 instead of Proposition 3 to show that the while-loop can be replaced by the limit of its finite approximations.

Similarly to Theorem 1 the equation we want to prove can be rewritten as:

$$
\mathrm{wlp} \llbracket C \rrbracket(f)(\sigma)=\int \check{f}\left(\mathbf{O}_{C}^{\sigma}(\theta)\right) \cdot \mathbf{S C}_{C}^{\sigma}(\theta) \mu_{\mathbb{S}}(d \theta)
$$

The proof goes as follows. Let $C=$ while $(\phi)\left\{C^{\prime}\right\}$ and $C^{n}=$ while ${ }^{n}(\phi)\left\{C^{\prime}\right\}$.

$$
\begin{aligned}
& \int \check{f}\left(\mathbf{O}_{C}^{\sigma}(\theta)\right) \cdot \mathbf{S C}_{C}^{\sigma}(\theta) \mu_{\mathbb{S}}(d \theta) \\
& \text { (Proposition } \mathbf{5}=\int \inf _{n} \check{f}\left(\mathbf{O}_{C^{n}}^{\sigma}(\theta)\right) \cdot \mathbf{S C}_{C^{n}}^{\sigma}(\theta) \mu_{\mathbb{S}}(d \theta) \\
& \text { (Beppo Levi's Theorem) }=\inf _{n} \int \check{f}\left(\mathbf{O}_{C^{n}}^{\sigma}(\theta)\right) \cdot \mathbf{S C}_{C^{n}}^{\sigma}(\theta) \mu_{\mathbb{S}}(d \theta) \\
&=\inf _{n}^{\mathrm{wlp}}\left\langle\phi, C^{\prime}\right\rangle \\
& \Phi_{f}^{n}(1)(\sigma) \\
& \text { (Kleene's Fixpoint Theorem) }=\mathrm{wlp} \llbracket \text { while }(\phi)\left\{C^{\prime}\right\} \rrbracket(f)(\sigma)
\end{aligned}
$$


In order to show that step $(*)$ is correct, we need to show that $\int \check{f}\left(\mathbf{O}_{C^{n}}^{\sigma}(\theta)\right)$. $\mathbf{S C}_{C^{n}}^{\sigma}(\theta) \mu_{\mathbb{S}}(d \theta)=\inf _{n} \underset{\left\langle\phi, C^{\prime}\right\rangle}{\text { wlp }} \Phi_{f}^{n}(1)(\sigma)$ for all $n$. This can be proven by induction on $n$; the proof is almost identical to the proof of $(*)$ from Theorem 11, When applying the Beppo Levi's Theorem, we used the fact that the sequence $\check{f}\left(\mathbf{O}_{C^{n}}^{\sigma}(\theta)\right) \cdot \mathbf{S C}_{C^{n}}^{\sigma}(\theta)$ is decreasing in $n$ (Proposition 6 ) and that $\int \check{f}\left(\mathbf{O}_{C^{0}}^{\sigma}(\theta)\right)$. $\mathbf{S C}_{C^{0}}^{\sigma}(\theta) \mu_{\mathbb{S}}(d \theta)<\infty$, which can be checked immediately.

\section{F Proving measurability}

The proofs of measurability are similar to [36], with the difference that we are working with an imperative language. In this section, we sketch the proofs of measurability of functions $\mathbf{O}_{C}^{\sigma}(\cdot)$ and $\mathbf{S C}_{C}^{\sigma}(\cdot, n)$, without going into the details, which are conceptually the same as in 36 .

\section{F.1 Measurability of single-step reduction}

Let us define:

$g\left(\theta, C, K, \sigma, \theta_{K}, n, w\right)=\left\{\begin{array}{l}\left(\theta^{\prime}, C^{\prime}, K^{\prime}, \sigma^{\prime}, \theta_{K}^{\prime}, n+1, w^{\prime}\right) \\ \quad \text { if }\left\langle\theta, C, K, \sigma, \theta_{K}, n, w\right\rangle \vdash\left\langle\theta^{\prime}, C^{\prime}, K^{\prime}, \sigma^{\prime}, \theta_{K}^{\prime}, n+1, w^{\prime}\right\rangle \\ \left(\theta, C, K, \downarrow, \theta_{K}, n+1,0\right) \quad \text { otherwise }\end{array}\right.$

We need to show that $g$ is measurable. The only interesting cases are (assign), which modifies state (we need to show $g$ is still continuous in this case) and (draw), which modifies both state and trace, and (seq) and (pop), which modify both the main trace and the trace for continuation.

We can show that $g$ is measurable by considering $g$ as a disjoint union of sub-functions defined on measurable subsets of combinations corresponding to given reduction rules (e.g. $g_{i f-t r u e}$ and $g_{i f-f a l s e}$ reducing conditional choices, $g_{\text {while-true }}$ and $g_{\text {while-false }}$ reducing while-loops, $g_{\text {sample }}$ reducing sampling statements etc.) and showing that each sub-function is measurable. The reasoning is very similar to the one presented in Appendix E.1 of [36], so we omit the full proof and only show measurability of sub-functions modifying states and infinite traces, which were not present in [36].

From continuity to measurability The easiest way of proving measurability of a function is often proving that this function is continuous as a function between the metric spaces which gave rise to the domain and codomain measurable spaces - by Corollary 2 continuity implies measurability. Moreover, Corollary 3 states that if a function $f$ between products of separable metric spaces is continuous with respect to the Manhattan products of metrics, then it is measurable with respect to products of the given measurable spaces. We will make heavy use of these results in the proofs below. 
Additional Borel $\sigma$-algebras In order to carry out the proofs, we need to define separable metric spaces on statements $C$, expressions $E$ and continuations $K$, which will induce Borel $\sigma$-algebras. These metrics are straightforward metrics on syntactic terms, similar to the metrics on lambda-terms in 36]. We omit the details, but these metrics would be defined so that $d_{C}\left(C_{1} ; C_{2}, C_{1}^{\prime} ; C_{2}^{\prime}\right)=$ $d_{C}\left(C_{1} ; C_{1}^{\prime}\right)+d_{C}\left(C_{2} ; C_{2}^{\prime}\right)$ and $d_{K}\left(C:: K, C^{\prime}:: K^{\prime}\right)=d_{C}\left(C ; C^{\prime}\right)+d_{K}\left(K, K^{\prime}\right)$ (where $d_{K}\left(K, K^{\prime}\right)=\infty$ if $K$ and $K^{\prime}$ have different lengths).

It is easy to check that all the above metric spaces are separable-for each of them, a dense subset can be obtained by replacing reals with rationals. All subspaces of separable metric spaces can also be shown to be separable.

We also need to define $\sigma$-algebras on step sizes $n$ and weights $w$-these will be the standard discrete $\sigma$-algebra on $\mathbb{Z}_{+}$and the Borel $\sigma$-algebra on $[0,1]$, respectively.

Measurability of (assign) We define:

$$
\begin{aligned}
g_{\text {assign }}\left(\theta, x:=E, K, \sigma, \theta_{K}, n, w\right)= & \left(\theta, \downarrow, K, \sigma[x \mapsto \sigma(E)], \theta_{K}, n+1, w\right) \\
= & \left(g_{\text {assign1 }}\left(\theta, x:=E, K, \sigma, \theta_{K}, n, w\right),\right. \\
& g_{\text {assign2 }}\left(\theta, x:=E, K, \sigma, \theta_{K}, n, w\right), \\
& \ldots, \\
& \left.g_{\text {assign }}\left(\theta, x:=E, K, \sigma, \theta_{K}, n, w\right)\right)
\end{aligned}
$$

where:

$$
\begin{aligned}
& g_{\text {assign } 1}\left(\theta, x:=E, K, \sigma, \theta_{K}, n, w\right)=\theta \\
& g_{\text {assign2 } 2}\left(\theta, x:=E, K, \sigma, \theta_{K}, n, w\right)=\downarrow \\
& g_{\text {assign3 } 3}\left(\theta, x:=E, K, \sigma, \theta_{K}, n, w\right)=K \\
& g_{\text {assign4 } 4}\left(\theta, x:=E, K, \sigma, \theta_{K}, n, w\right)=\sigma[x \mapsto \sigma(E)] \\
& g_{\text {assign } 5}\left(\theta, x:=E, K, \sigma, \theta_{K}, n, w\right)=\theta_{K} \\
& g_{\text {assign } 6}\left(\theta, x:=E, K, \sigma, \theta_{K}, n, w\right)=n+1 \\
& g_{\text {assign } 7}\left(\theta, x:=E, K, \sigma, \theta_{K}, n, w\right)=w
\end{aligned}
$$

Lemma 47. $g_{\text {assign }}$ is measurable.

Proof. The functions $g_{\text {assign } 1}, g_{\text {assign } 3}, g_{\text {assign } 5}, g_{\text {assign } 7}$ are simple projections, so they are trivially measurable. The function $g_{a s s i g n} 2$ is a constant function, so it is also measurable. Function $g_{a s s i g n} 4$ is a composition of a function returning the tuple $(x, \sigma, \sigma(E))$ from the configuration, which can easily be shown measurable (projections are measurable, the function extracting $E$ from $x:=E$ can be shown continuous and substitution $\sigma(E)$ is measurable by assumption), and the state update function, which is measurable by Lemma 14. Function $g_{\text {assign } 6}$ is a composition of a projection (returning the sixth component $n$ from a tuple) and a function adding 1 to a number, which is continuous and measurable.

Hence, $g_{\text {assign }}$ is measurable, as all its components are measurable. 
Measurability of (draw) Let us define:

$$
\begin{aligned}
g_{\text {draw }}\left(\left(\theta, x: \approx U, K, \sigma, \theta_{K}, n, w\right)\right)= & \left(\pi_{R}(\theta), \downarrow, K, \sigma\left[x \mapsto \pi_{U}\left(\pi_{L}(\theta)\right)\right], \theta_{K}, n+1, w\right) \\
= & \left(g_{\text {draw } 1}\left(\theta, x:=E, K, \sigma, \theta_{K}, n, w\right),\right. \\
& g_{\text {draw2 }}\left(\theta, x:=E, K, \sigma, \theta_{K}, n, w\right), \\
& \cdots, \\
& \left.g_{\text {draw } \gamma}\left(\theta, x:=E, K, \sigma, \theta_{K}, n, w\right)\right)
\end{aligned}
$$

where:

$$
\begin{aligned}
& g_{\text {draw } 1}\left(\theta, x:=E, K, \sigma, \theta_{K}, n, w\right)=\pi_{R}(\theta) \\
& g_{\text {draw } 2}\left(\theta, x:=E, K, \sigma, \theta_{K}, n, w\right)=\downarrow \\
& g_{\text {draw } 3}\left(\theta, x:=E, K, \sigma, \theta_{K}, n, w\right)=K \\
& g_{\text {draw } 4}\left(\theta, x:=E, K, \sigma, \theta_{K}, n, w\right)=\sigma\left[x \mapsto \pi_{U}\left(\pi_{L}(\theta)\right)\right] \\
& g_{\text {draw3 } 3}\left(\theta, x:=E, K, \sigma, \theta_{K}, n, w\right)=\theta_{K} \\
& g_{\text {draw } 6}\left(\theta, x:=E, K, \sigma, \theta_{K}, n, w\right)=n+1 \\
& g_{\text {draw } 7}\left(\theta, x:=E, K, \sigma, \theta_{K}, n, w\right)=w
\end{aligned}
$$

Lemma 48. $g_{\text {assign }}$ is measurable.

Proof. We only need to show the measurability of $g_{\text {draw } 1}$ and $g_{\text {draw }}$, as the other functions are identical to the ones used in the definition of $g_{\text {assign }}$.

The function $g_{\text {draw1 }}$ is a composition of the projection returning the first component $\theta$ of the configuration, and the function $\pi_{R}$, which is measurable by the axiomatisation of the entropy space, so it is measurable.

Function $g_{\text {draw } 4}$ is measurable by the same argument as $g_{\text {assign } 4}$, except that the measurable evaluation $\sigma(E)$ is replaced by $\pi_{U}\left(\pi_{L}(\theta)\right)$, which as a composition of two measurable (by assumption) functions and the measurable projection returning $\theta$ is also measurable.

Measurability of (seq) and (pop) Define:

$$
\begin{aligned}
g_{\text {seq }}\left(\left(\theta, C_{1} ; C_{2}, K, \sigma, \theta_{K}, n, w\right)\right)= & \left(\pi_{L}(\theta), C_{1}, C_{2}:: K, \sigma, \pi_{R}(\theta):: \theta_{K}, n+1, w\right) \\
= & \left(g_{\text {seq } 1}\left(\theta, x:=E, K, \sigma, \theta_{K}, n, w\right),\right. \\
& g_{\text {seq2 }}\left(\theta, x:=E, K, \sigma, \theta_{K}, n, w\right), \\
& \ldots, \\
& \left.g_{\text {seq } 7}\left(\theta, x:=E, K, \sigma, \theta_{K}, n, w\right)\right)
\end{aligned}
$$


where:

$$
\begin{aligned}
& g_{\text {seq } 1}\left(\theta, C_{1} ; C_{2}, K, \sigma, \theta_{K}, n, w\right)=\pi_{L}(\theta) \\
& g_{\text {seq2 }}\left(\theta, C_{1} ; C_{2}, K, \sigma, \theta_{K}, n, w\right)=C_{1} \\
& g_{\text {seq3 }}\left(\theta, C_{1} ; C_{2}, K, \sigma, \theta_{K}, n, w\right)=C_{2}:: K \\
& g_{\text {seq4 }}\left(\theta, C_{1} ; C_{2}, K, \sigma, \theta_{K}, n, w\right)=\sigma \\
& g_{\text {seq } 5}\left(\theta, C_{1} ; C_{2}, K, \sigma, \theta_{K}, n, w\right)=\pi_{R}(\theta):: \theta_{K} \\
& g_{\text {seq } 6}\left(\theta, C_{1} ; C_{2}, K, \sigma, \theta_{K}, n, w\right)=n+1 \\
& g_{\text {seq } 7}\left(\theta, C_{1} ; C_{2}, K, \sigma, \theta_{K}, n, w\right)=w
\end{aligned}
$$

Lemma 49. $g_{\text {seq }}$ is measurable.

Proof. The function $g_{\text {seq } 1}$ is measurable as a composition of projection and a function measurable by assumption. The metrics $d_{C}$ and $d_{K}$ on statements and continuations (whose formal definitions are omitted) satisfy $d_{C}\left(C_{1} ; C_{2}, C_{1}^{\prime} ; C_{2}^{\prime}\right)=$ $d_{C}\left(C_{1} ; C_{1}^{\prime}\right)+d_{C}\left(C_{2} ; C_{2}^{\prime}\right)$ and $d_{K}\left(C:: K, C^{\prime}:: K^{\prime}\right)=d_{C}\left(C ; C^{\prime}\right)+d_{K}\left(K, K^{\prime}\right)$, which makes it easy to show that $g_{\text {seq2 }}$ and $g_{\text {seq3 }}$ are measurable, as compositions of projections and continuous functions. Meanwhile, $g_{\text {seq } 5}$ is composed from measurable projections and the functions $\pi_{R}$ and (::), measurable by assumption, so it is measurable.

The proof of measurability of (pop) is analogous.

\section{F.2 Measurability of $\mathrm{O}_{C}^{\sigma}(\cdot)$ and $\operatorname{SC}_{C}^{\sigma}(\cdot, n)$}

Once we have proven the measurability of state updates, the proof of Lemma 9 (measurability of $\mathbf{O}_{C}^{\sigma}(\cdot)$ ) is analogous to the proof of Lemma 92 in [5].

The proof of measurability of $\mathbf{S C}_{C}^{\sigma}(\cdot, n)$ is even simpler-for each fixed $n$, we can represent $\mathbf{S C}_{C}^{\sigma}(\cdot, n)$ as an $n$-fold composition of $g$, followed by a projection returning the weight $w$ from the configuration. The projection is obviously continuous, and so measurable. Since a composition of measurable functions is measurable, this shows that $\mathbf{S C}_{C}^{\sigma}(\cdot, n)$ is measurable. 


\section{Bibliography}

[1] S. Abramsky and A. Jung. Domain theory. In S. Abramsky, D. M. Gabbay, and T. S. E. Maibaum, editors, Handbook of Logic in Computer Science (Vol. 3), pages 1-168. Oxford University Press, Inc., 1994.

[2] R. J. Aumann. Borel structures for function spaces. Illinois J. Math., 5(4):614-630, 121961.

[3] B. Bichsel, T. Gehr, and M. T. Vechev. Fine-grained semantics for probabilistic programs. In A. Ahmed, editor, Programming Languages and Systems - 27th European Symposium on Programming, ESOP 2018, Held as Part of the European Joint Conferences on Theory and Practice of Software, ETAPS 2018, Thessaloniki, Greece, April 14-20, 2018, Proceedings, volume 10801 of Lecture Notes in Computer Science, pages 145-185. Springer, 2018.

[4] P. Billingsley. Probability and Measure. Wiley, 3rd edition, 1995.

[5] J. Borgström, U. Dal Lago, A. D. Gordon, and M. Szymczak. A lambdacalculus foundation for universal probabilistic programming. In J. Garrigue, G. Keller, and E. Sumii, editors, Proceedings of the 21st ACM SIGPLAN International Conference on Functional Programming, ICFP 2016, Nara, Japan, September 18-22, 2016, pages 33-46. ACM, 2016.

[6] J. Borgström, A. D. Gordon, M. Greenberg, J. Margetson, and J. V. Gael. Measure transformer semantics for Bayesian machine learning. Logical Methods in Computer Science, 9(3), 2013.

[7] K. Chatterjee, P. Novotný, and D. Zikelic. Stochastic invariants for probabilistic termination. In G. Castagna and A. D. Gordon, editors, Proceedings of the 44th ACM SIGPLAN Symposium on Principles of Programming Languages, POPL 2017, Paris, France, January 18-20, 2017, pages 145-160. $\mathrm{ACM}, 2017$.

[8] R. Culpepper and A. Cobb. Contextual equivalence for probabilistic programs with continuous random variables and scoring. In H. Yang, editor, Programming Languages and Systems - 26th European Symposium on Programming, ESOP 2017, Held as Part of the European Joint Conferences on Theory and Practice of Software, ETAPS 2017, Uppsala, Sweden, April 2229, 2017, Proceedings, volume 10201 of Lecture Notes in Computer Science, pages 368-392. Springer, 2017.

[9] F. Dahlqvist and D. Kozen. Semantics of higher-order probabilistic programs with conditioning. Proc. ACM Program. Lang., 4(POPL):57:1-57:29, 2020.

[10] N. D. Goodman, V. K. Mansinghka, D. M. Roy, K. Bonawitz, and J. B. Tenenbaum. Church: a language for generative models. In D. A. McAllester and P. Myllymäki, editors, UAI 2008, Proceedings of the 24th Conference in Uncertainty in Artificial Intelligence, Helsinki, Finland, July 9-12, 2008, pages 220-229. AUAI Press, 2008.

[11] N. D. Goodman and A. Stuhlmüller. The Design and Implementation of Probabilistic Programming Languages. http://dippl.org, 2014. 
[12] N. D. Goodman, J. B. Tenenbaum, and T. P. Contributors. Probabilistic Models of Cognition. http://probmods.org/v2, 2016.

[13] F. Gretz, J. Katoen, and A. McIver. Operational versus weakest preexpectation semantics for the probabilistic guarded command language. Perform. Eval., 73:110-132, 2014.

[14] E. Habil. Double sequences and double series. IUG Journal of Natural Studies, 14(1), 012006.

[15] C. Heunen, O. Kammar, S. Staton, and H. Yang. A convenient category for higher-order probability theory. In 32nd Annual ACM/IEEE Symposium on Logic in Computer Science, LICS 2017, Reykjavik, Iceland, June 20-23, 2017, pages 1-12. IEEE Computer Society, 2017.

[16] D. Huang and G. Morrisett. An application of computable distributions to the semantics of probabilistic programming languages. In P. Thiemann, editor, Programming Languages and Systems - 25th European Symposium on Programming, ESOP 2016, Held as Part of the European Joint Conferences on Theory and Practice of Software, ETAPS 2016, Eindhoven, The Netherlands, April 2-8, 2016, Proceedings, volume 9632 of Lecture Notes in Computer Science, pages 337-363. Springer, 2016.

[17] G. Hutton. Introduction to Domain Theory, 1994. Lecture notes available online at http://www.cs.nott.ac.uk/ pszgmh/domains.html.

[18] T. Icard. Beyond almost-sure termination. In G. Gunzelmann, A. Howes, T. Tenbrink, and E. J. Davelaar, editors, Proceedings of the 39th Annual Meeting of the Cognitive Science Society, CogSci 2017, London, UK, 16-29 July 2017. cognitivesciencesociety.org, 2017.

[19] H. Ishwaran and L. F. James. Gibbs sampling methods for stick-breaking priors. Journal of the American Statistical Association, 96(453):161-173, 2001.

[20] N. Jansen, B. L. Kaminski, J. Katoen, F. Olmedo, F. Gretz, and A. McIver. Conditioning in probabilistic programming. In D. R. Ghica, editor, The 31st Conference on the Mathematical Foundations of Programming Semantics, MFPS 2015, Nijmegen, The Netherlands, June 22-25, 2015, volume 319 of Electronic Notes in Theoretical Computer Science, pages 199-216. Elsevier, 2015.

[21] B. L. Kaminski. Advanced Weakest Precondition Calculi for Probabilistic Programs. PhD thesis, RWTH Aachen University, Feb. 2019.

[22] B. L. Kaminski, J. Katoen, C. Matheja, and F. Olmedo. Weakest precondition reasoning for expected runtimes of randomized algorithms. J. ACM, 65(5):30:1-30:68, 2018.

[23] J. Katoen. The probabilistic model checking landscape. In M. Grohe, E. Koskinen, and N. Shankar, editors, Proceedings of the 31st Annual ACM/IEEE Symposium on Logic in Computer Science, LICS '16, New York, NY, USA, July 5-8, 2016, pages 31-45. ACM, 2016.

[24] D. Kozen. Semantics of probabilistic programs. J. Comput. Syst. Sci., 22(3):328-350, 1981.

[25] A. McIver and C. Morgan. Abstraction, Refinement and Proof for Probabilistic Systems. Monographs in computer science. Springer, 2005. 
[26] C. Morgan and A. McIver. Unifying wp and wlp. Inf. Process. Lett., 59(3):159-163, 1996.

[27] C. Morgan, A. McIver, and K. Seidel. Probabilistic predicate transformers. ACM Trans. Program. Lang. Syst., 18(3):325-353, 1996.

[28] A. V. Nori, C. Hur, S. K. Rajamani, and S. Samuel. R2: an efficient MCMC sampler for probabilistic programs. In C. E. Brodley and P. Stone, editors, Proceedings of the Twenty-Eighth AAAI Conference on Artificial Intelligence, July 27-31, 2014, Québec City, Québec, Canada, pages 2476-2482. AAAI Press, 2014.

[29] F. Olmedo, F. Gretz, N. Jansen, B. L. Kaminski, J. Katoen, and A. McIver. Conditioning in probabilistic programming. ACM Trans. Program. Lang. Syst., 40(1):4:1-4:50, 2018.

[30] F. Olmedo, B. L. Kaminski, J. Katoen, and C. Matheja. Reasoning about recursive probabilistic programs. In M. Grohe, E. Koskinen, and N. Shankar, editors, Proceedings of the 31st Annual ACM/IEEE Symposium on Logic in Computer Science, LICS '16, New York, NY, USA, July 5-8, 2016, pages 672-681. ACM, 2016.

[31] S. Park, F. Pfenning, and S. Thrun. A probabilistic language based on sampling functions. ACM Trans. Program. Lang. Syst., 31(1), Dec. 2008.

[32] N. Saheb-Djahromi. Probabilistic LCF. In J. Winkowski, editor, Mathematical Foundations of Computer Science 1978, Proceedings, 7th Symposium, Zakopane, Poland, September 4-8, 1978, volume 64 of Lecture Notes in Computer Science, pages 442-451. Springer, 1978.

[33] A. Ścibior, O. Kammar, and Z. Ghahramani. Functional programming for modular Bayesian inference. Proc. ACM Program. Lang., 2(ICFP):83:183:29, 2018.

[34] R. M. Solovay. A model of set-theory in which every set of reals is lebesgue measurable. Annals of Mathematics, 92(1):1-56, 1970.

[35] S. Staton, H. Yang, F. Wood, C. Heunen, and O. Kammar. Semantics for probabilistic programming: Higher-order functions, continuous distributions, and soft constraints. In Proceedings of the 31st Annual ACM/IEEE Symposium on Logic in Computer Science, LICS '16, pages 525-534, New York, NY, USA, 2016. ACM.

[36] M. Szymczak. Programming Language Semantics as a Foundation for Bayesian Inference. PhD thesis, University of Edinburgh, 2018.

[37] N. Toronto, J. McCarthy, and D. V. Horn. Running probabilistic programs backwards. In J. Vitek, editor, Programming Languages and Systems - 24th European Symposium on Programming, ESOP 2015, Held as Part of the European Joint Conferences on Theory and Practice of Software, ETAPS 2015, London, UK, April 11-18, 2015. Proceedings, volume 9032 of Lecture Notes in Computer Science, pages 53-79. Springer, 2015.

[38] M. Vákár, O. Kammar, and S. Staton. A domain theory for statistical probabilistic programming. Proc. ACM Program. Lang., 3(POPL):36:1$36: 29,2019$.

[39] M. Wand, R. Culpepper, T. Giannakopoulos, and A. Cobb. Contextual equivalence for a probabilistic language with continuous random variables and recursion. Proc. ACM Program. Lang., 2(ICFP):87:1-87:30, 2018. 